\title{
Liquidity Risks, Transaction costs and Online Portfolio Selection is
}

\author{
Youngmin $\mathrm{Ha}^{\mathrm{a}}$ and Hai Zhang ${ }^{\mathrm{b}}$

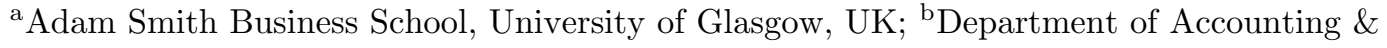 \\ Finance, Strathclyde Business School, UK
}

(First draft: April 11, 2016; this draft: April 4, 2018)

\begin{abstract}
The performance of online (sequential) portfolio selection (OPS), which rebalances a portfolio in every period (e.g. daily or weekly) in order to maximise the portfolio's expected terminal wealth in the long run, has been overestimated by the ideal assumption of unlimited market liquidity (i.e. no market impact costs). Therefore, a new transaction cost factor model that considers both market impact costs, estimated from limit order book data, and proportional transaction costs (e.g. brokerage commissions or transaction taxes in a fixed percentage) has been proposed in this paper to measure existing OPS strategies performance in a more practical way as well as to develop a more effective OPS method. Backtesting results from the historical limit order book (LOB) data of NASDAQ-traded stocks show both the performance deterioration of existing OPS methods by the market impact costs and the superiority of our proposed OPS method in the environment of limited market liquidity.
\end{abstract}

Keywords: online portfolio selection; transaction cost; market impact cost; liquidity risks; LOB

JEL Classification: G11, C63

\section{Introduction}

Online (sequential) portfolio selection (OPS henceforth) aims to maximise the portfolio's expected terminal wealth in the long run and differs from single-period (Markowitz 1959) or multi-period (Dantzig and Infanger 1993, Ben-Tal et al. 2000, Li and Ng 2000, Steinbach 2001) mean-variance portfolio selection (MVPS). To be more specific, OPS makes minimal statistical assumptions about the behaviour of the stock market (e.g. stationarity and ergodicity), whereas MVPS assumes a lognormal distribution of stock returns (Markowitz 1959). Furthermore, OPS aims for higher expected terminal wealth without considering the variance (risk), but MVPS makes trade-off between the mean (expected wealth) and variance (risk).

Both OPS and multi-period MVPS rebalance a portfolio periodically before an investor obtains the final reward. Two key differences between them are i) the uncertainty of input data for portfolio rebalancing, e.g. OPS uses deterministic data (e.g. historical stock returns) while multi-period MVPS employs stochastic data (e.g. mean and covariance of stock returns' distribution), and ii) the frequency of portfolio rebalancing, for example, OPS rebalances a portfolio at the same frequency as the input data, but multi-period MVPS rebalances at a lower frequency than the input data.

\footnotetext{
This paper is a modified version of part of Younmin Ha's PhD thesis submitted to the University of Glasgow (http: //theses.gla.ac.uk/8558/).

CONTACT Hai Zhang. Email: hai.zhang@strath.ac.uk
} 
OPS differs from portfolio optimisation using a risk measure based either on Value-at-Risk (VaR) or on conditional VaR $(\mathrm{CVaR})$ though neither of them nor OPS assumes any specific distribution of stock returns (Gaivoronski and Pflug 2005). However, OPS uses stock return time series spanning between the first day of investment and the current day, whereas portfolio optimisation using either VaR or CVaR uses the finite samples in the left tail of historical stock returns spanning before the first day of investment.

OPS directly optimises a portfolio in terms of the long-term investment without forecasting ( $\mathrm{Li}$ and Hoi 2014), and it differs from the previous studies of prediction-based portfolio selection (Freitas et al. 2009, Otranto 2010, Brown and Smith 2011, Ferreira and Santa-Clara 2011, Gârleanu and Pedersen 2013, DeMiguel et al. 2014, Palczewski et al. 2015), which i) forecasts the expected values or covariance matrix of stock returns ${ }^{1}$ and ii) uses the mean-variance optimisation. ${ }^{2}$ Therefore, OPS neither suffers from the difficulty of the prediction nor uses in-sample and out-of-sample tests.

Almost all existing OPS methods (Blum and Kalai 1999, Györfi and Vajda 2008, Kozat and Singer 2011, Bean and Singer 2012, Györfi and Walk 2012, Tunc et al. 2013, Das et al. 2013, 2014) consider only the proportional transaction costs and none of them has considered the liquidity risks or the market impact costs, a common feature of financial markets. Therefore, the aim of this paper is to develop a new model of transaction cost factor (TCF; this will be explained in Section 5) by considering LOBs of stocks of which a portfolio consists. Almost all the previous methods of OPS (these will be reviewed in Section 3) simply assumed that an investor can buy or sell any quantities of stocks, which in turn makes these OPS methods impractical. The ideal assumption of unlimited liquidity or no liquidity risks in financial markets when rebalancing a portfolio no doubt overestimates the performance of OPS. To overcome these problems, this paper has proposed a more practical OPS method which relaxes the unlimited liquidity assumption by using LOB data, which reflects the liquidity risks of assets and has the superior performance as well.

The main contributions of this paper are:

- in Section 5.2 and Section 5.3, to propose the new TCF model which reflects LOBs as well as a fixed percentage fee;

- in Section 8, to present the backtesting results of a comparison among OPS methods (including the proposed method) by using the real-world data (historical NASDAQ LOB data).

The rest of this paper is organised as follows: Section 2 lists mathematical notations. Section 3 describes the existing methods of OPS. Section 4 includes the motivation of this paper and the review of the mathematical formulation of market impact costs (MICs) as a function of order size. Section 5 reviews a TCF model without MICs and proposes the new TCF model with MICs. Section 6 reviews a log-optimal portfolio, one of the OPS methods, which is the basis of the proposed method. Section 7 describes the proposed OPS method. Section 8 demonstrates the performance of OPS methods including the proposed method by computer simulations (backtesting). Section 9 gives the conclusion.

\section{Mathematical setup}

The following notations are used in this paper:

- $\boldsymbol{b}_{n}=\left[\begin{array}{llll}b_{n}^{(1)} & b_{n}^{(2)} & \ldots & b_{n}^{(d)}\end{array}\right]^{\mathrm{T}}$ is a portfolio vector of $d$ risky assets (there is no risk-free asset in the portfolio) at the $n$-th period (trading occurs in a fixed interval such as a day or a week

1 Dynamic portfolio selection with transaction costs prevents too much trading by using multi-period prediction in the time horizon from $t+1$ to $t+h$ (Brown and Smith 2011), and from $t+1$ to $\infty$ (Gârleanu and Pedersen 2013), where $t$ is the current period. Both the methods show better backtesting results than their benchmarks with single-period prediction of $t+1$.

2 Brown and Smith (2011), Palczewski et al. (2015) used risk-averse utility functions instead of the mean-variance portfolio. In addition, DeMiguel et al. (2014) constructed an arbitrage (zero-cost) portfolio, creating a zero net value, as well as the mean-variance portfolio. 


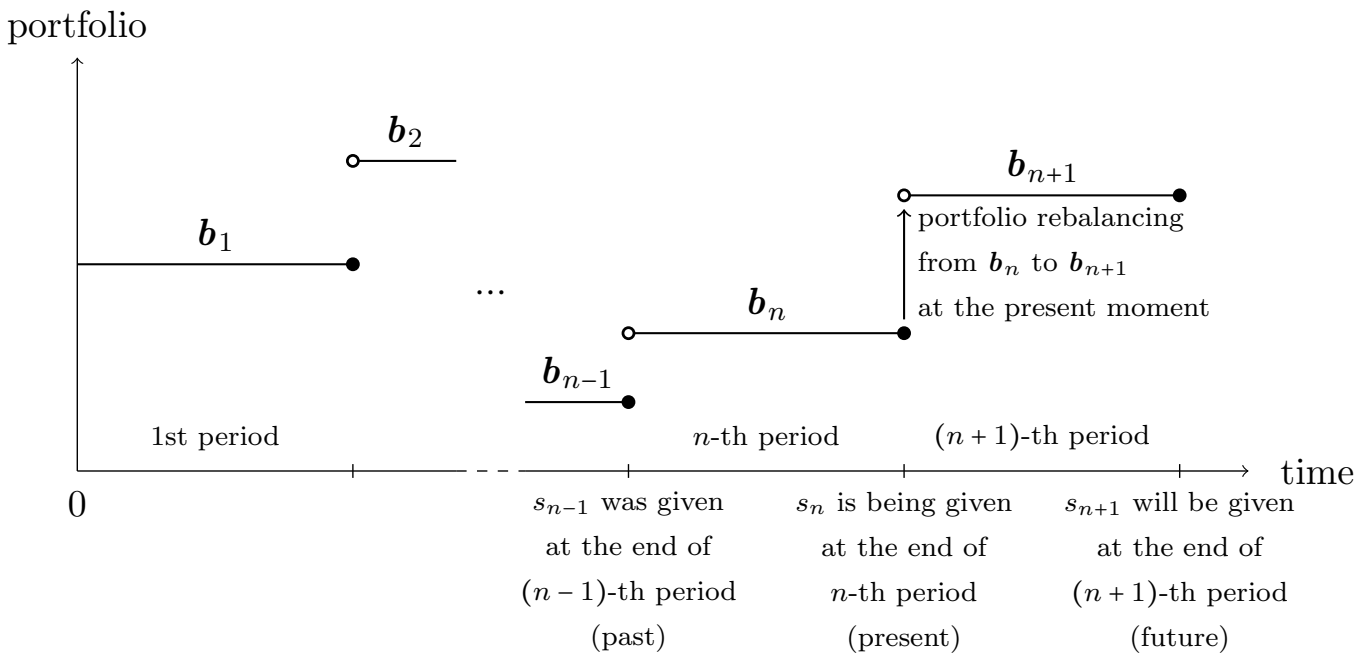

Figure 1. Timeline of trading. A portfolio is rebalanced at the end of every period, where an asset's price is given at the end of every period. The end of $n$-th period is the present moment for rebalancing.

at the end of every period as shown in Figure 1 ), where $n \in \mathbb{Z}_{\geq 1}, b_{n}^{(j)} \in \mathbb{R}_{\geq 0}$ (i.e. neither short selling nor buying stocks on margin is permitted), and $\sum_{j=1}^{d} b_{n}^{(j)}=1$ (i.e. $b_{n}^{(j)}$ is the proportion of a portfolio invested in asset $j \in\{1,2, \ldots, d\}$ at the $n$-th period). Hence, $\boldsymbol{b}_{n} \in \Delta^{d-1}$, where $\Delta^{d-1}=\left\{\left[\begin{array}{llll}b^{(1)} & b^{(2)} & \ldots & b^{(d)}\end{array}\right]^{\mathrm{T}} \in \mathbb{R}_{\geq 0}^{d} \mid \sum_{j=1}^{d} b^{(j)}=1\right\}$ is the standard $(d-1)$-simplex.

- $\boldsymbol{b}_{1}=[1 / d 1 / d \ldots 1 / d]^{\mathrm{T}}$ is an initial portfolio vector.

- $s_{n}^{(j)}$ is the price of asset $j$ at the end of the $n$-th period (see Figure 1).

- $x_{n}^{(j)}=\frac{s_{n}^{(j)}}{s_{n-1}^{(j)}}$ is a price relative of asset $j$ at the end of the $n$-th period.

- $\boldsymbol{x}_{n}=\left[\begin{array}{llll}x_{n}^{(1)} & x_{n}^{(2)} & \ldots & x_{n}^{(d)}\end{array}\right]^{\mathrm{T}} \in \mathbb{R}_{>0}^{d}$ is a market vector at the end of the $n$-th period.

- $\boldsymbol{x}_{i: j}=\left[\begin{array}{c}\boldsymbol{x}_{i} \\ \boldsymbol{x}_{i+1} \\ \vdots \\ \boldsymbol{x}_{j}\end{array}\right] \in \mathbb{R}_{>0}^{d(j-i+1)}$ is the array of the market vectors, where $i \leq j$.

\section{Review of the existing methods of online portfolio selection}

The two categories of OPS, classified according to whether considering transaction costs (TCs) or not, are reviewed in the following subsections.

\subsection{Online portfolio selection without transaction costs $^{1}$}

The most basic benchmark of OPS is a best constant rebalanced portfolio (BCRP), whose portfolio vector is

$$
\boldsymbol{b}^{*}=\underset{\boldsymbol{b} \in \Delta^{d-1}}{\arg \max } \prod_{i=1}^{T}\left\langle\boldsymbol{b}, \boldsymbol{x}_{i}\right\rangle
$$

1 A detailed survey of OPS without TCs was carried out by Li and Hoi (2014), and Das (2014, pp. 22-29). 
where $T$ is the last period of trading, and $\langle\cdot, \cdot\rangle$ denotes the inner product. This is a hindsight strategy that can only be calculated with complete market vectors from the first period to the last period in the future $\boldsymbol{x}_{1: T}$; hence, BCRP is unimplementable as an investment strategy but available only for benchmark.

A universal portfolio (UP) is the beginning of OPS and performs asymptotically as well as BCRP under the assumption that stock returns are a stationary ergodic time series (Cover 1991). The portfolio vector of UP of the next period $n+1$ ( $n$ is the current period as shown in Figure 1 ) is

$$
\boldsymbol{b}_{n+1}=\frac{\int_{\Delta^{d-1}} \boldsymbol{b} S_{n}\left(\boldsymbol{b}, \boldsymbol{x}_{1: n}\right) f(\boldsymbol{b}) d \boldsymbol{b}}{\int_{\Delta^{d-1}} S_{n}\left(\boldsymbol{b}, \boldsymbol{x}_{1: n}\right) f(\boldsymbol{b}) d \boldsymbol{b}},{ }^{1}
$$

where $S_{n}\left(\boldsymbol{b}, \boldsymbol{x}_{1: n}\right)=S_{0} \prod_{i=1}^{n}\left\langle\boldsymbol{b}, \boldsymbol{x}_{i}\right\rangle$ is wealth at the end of the $n$-th period with an initial wealth $S_{0}$, and $f(\cdot)$ is the probability density function (PDF) of the Dirichlet distribution with the $d$-dimensional concentration parameter vector $\left[\begin{array}{llll}1 / 2 & 1 / 2 & \ldots & 1 / 2\end{array}\right]^{\mathrm{T}}$. The UP strategy is a follow-thewinner approach according to $\mathrm{Li}$ and Hoi $(2014)$ as it increases the relative weights $S_{n}\left(\boldsymbol{b}, \boldsymbol{x}_{1: n}\right)$ of more successful assets in the past. In addition, Cover and Ordentlich (1996) extended UP (Cover 1991) to UP with side information, which uses additional information concerning the stock market (e.g. a series of trading signals).

A non-parametric (i.e. the distribution of the market vector is unknown) kernel-based sequential log-optimal investment strategy ${ }^{2}$ guarantees an optimal asymptotic growth rate of capital under minimal assumptions on the behaviour of the market (i.e. daily price relatives $\boldsymbol{x}_{i}$ are $K$-th order stationary Markov processes) (Györfi et al. 2006). Its portfolio vector of the next period is

$$
\boldsymbol{b}_{n+1}=\frac{\sum_{k=1}^{K} \sum_{l=1}^{L} \boldsymbol{h}^{(k, l)}\left(\boldsymbol{x}_{1: n}\right) S_{n}\left(\boldsymbol{h}^{(k, l)}\left(\boldsymbol{x}_{1: n}\right), \boldsymbol{x}_{1: n}\right)}{\sum_{k=1}^{K} \sum_{l=1}^{L} S_{n}\left(\boldsymbol{h}^{(k, l)}\left(\boldsymbol{x}_{1: n}\right), \boldsymbol{x}_{1: n}\right)},
$$

where $S_{n}\left(\boldsymbol{h}^{(k, l)}\left(\boldsymbol{x}_{1: n}\right), \boldsymbol{x}_{1: n}\right)=S_{0} \prod_{i=1}^{n}\left\langle\boldsymbol{h}^{(k, l)}\left(\boldsymbol{x}_{1: n}\right), \boldsymbol{x}_{i}\right\rangle$ is wealth at the end of the $n$-th period with an initial wealth $S_{0}$ and expert $\boldsymbol{h}^{(k, l)}(\cdot)$ : i.e. the higher wealth $S_{n}\left(\boldsymbol{h}^{(k, l)}(\cdot), \cdot\right)$; the greater weight on $\boldsymbol{b}_{n+1}$. The portfolio vector of the expert $\boldsymbol{h}^{(k, l)}(\cdot)$ from the series of past and current market vectors $\boldsymbol{x}_{1: n}$ is

$$
\boldsymbol{h}^{(k, l)}\left(\boldsymbol{x}_{1: n}\right)=\left\{\begin{array}{cl}
\boldsymbol{b}_{1}, & \text { if } J_{n}=\varnothing \\
\underset{\boldsymbol{b} \in \Delta^{d-1}}{\arg \max } \sum_{i \in J_{n}} \ln \left\langle\boldsymbol{b}, \boldsymbol{x}_{i+1}\right\rangle,{ }^{3} & \text { otherwise }
\end{array}\right.
$$

where $J_{n}$ is the locations of matches:

$$
J_{n}=\left\{k \leq i \leq n-1:\left\|\boldsymbol{x}_{i-k+1: i}-\boldsymbol{x}_{n-k+1: n}\right\| \leq l / c\right\} .
$$

This strategy is classified as a pattern-matching-based approach by Li and Hoi (2014) as it finds the matching in Euclidean space between the past market vectors $\boldsymbol{x}_{i-k+1: i}$ and the most recent market vectors $\boldsymbol{x}_{n-k+1: n}$. According to their numerical results, it outperformed UP (Cover 1991) although its performance (i.e. the terminal wealth) varies by the choice of the three free parameters: $K, L$, and $c$ in (3) and (5).

\footnotetext{
The mathematical proof of (2) is more easily explained in (Cover and Thomas 2006, Chapter 16) than (Cover 1991).

2 Log-optimal portfolio is explained in Section 6.

$3 \arg \min _{\boldsymbol{b} \in \Delta^{d-1}}\left(-\sum_{i \in J_{n}} \ln \left\langle\boldsymbol{b}, \boldsymbol{x}_{i+1}\right\rangle\right)$ is a convex optimisation problem (proof is in Appendix B), which means any local solution is guaranteed to be a global solution.
} 
The computation time to calculate $\boldsymbol{h}^{(k, l)}(\cdot)$ in (4) was decreased by transforming the constrained (i.e. $\boldsymbol{b} \in \Delta^{d-1}$ in (4)) nonlinear (i.e. the log function in (4)) programming to the constrained quadratic programming (Györfi et al. 2007):

$$
\overline{\boldsymbol{h}}^{(k, l)}\left(\boldsymbol{x}_{1: n}\right)=\underset{\boldsymbol{b} \in \Delta^{d-1}}{\arg \max } \sum_{i \in J_{n}}\left(\left\langle\boldsymbol{b}, \boldsymbol{x}_{i+1}\right\rangle-1-\frac{1}{2}\left(\left\langle\boldsymbol{b}, \boldsymbol{x}_{i+1}\right\rangle-1\right)^{2}\right), J_{n} \neq \varnothing,
$$

(i.e. the second-order Taylor expansion of the log function was used). Moreover, the computation time of the optimisation problem of (6) was reduced further by Györfi et al. (2007) by rewriting (6) as

$$
\overline{\boldsymbol{h}}^{(k, l)}\left(\boldsymbol{x}_{1: n}\right)=\underset{\boldsymbol{b} \in \Delta^{d-1}}{\arg \max }(\langle\boldsymbol{b}, \boldsymbol{m}\rangle-\langle\boldsymbol{b}, \boldsymbol{\Sigma} \boldsymbol{b}\rangle),{ }^{1} J_{n} \neq \varnothing,
$$

where $\boldsymbol{m}$ is the $d$-dimensional column vector ( 1 denotes the all-ones column vector):

$$
m=\sum_{i \in J_{n}}\left(x_{i+1}-1\right)
$$

and $\boldsymbol{\Sigma}$ is the $d \times d$ matrix:

$$
\Sigma=\frac{1}{2} \sum_{i \in J_{n}}\left(x_{i+1}-1\right)\left(x_{i+1}-1\right)^{\mathrm{T}}
$$

As a result, if we calculate $\boldsymbol{m}$ and $\boldsymbol{\Sigma}$ beforehand, the complexity of the optimisation problem of (7) does not depend on the number of matches $\left|J_{n}\right|$ (i.e. the size of $\boldsymbol{m}$ and $\boldsymbol{\Sigma}$ is fixed even if $\left|J_{n}\right|$ in (7) changes), while that of (6) does.

Györfi et al. (2008b) also assumed that daily price relatives are $K$-th order stationary Markov process as the same as (Györfi et al. 2006) and made a nearest-neighbour(NN)-based investment strategy with two free parameters: $K$ and $L$. The difference between the two strategies is only the way to find the locations of matching $J_{n}$ in (5): instead of the threshold of the distance $l / c$ in (5), the criterion of NN in Euclidean space is used as

$$
J_{n}=\left\{k \leq i \leq n-1 \mid \boldsymbol{x}_{i-k+1: i} \text { is among the } \hat{l} \text { NNs of } \boldsymbol{x}_{n-k+1: n} \text { in } \boldsymbol{x}_{1: k}, \boldsymbol{x}_{2: k+1}, \ldots, \boldsymbol{x}_{n-k: n-1}\right\},
$$

where $\hat{l}=\left\lfloor p_{l} n\right\rfloor$, and $p_{l}=0.02+0.5 \frac{l-1}{L-1}$, specified by Györfi et al. (2008a). Their experimental results showed that the NN-based method (Györfi et al. 2008b) outperforms the kernel-based method (Györfi et al. 2006) in terms of the terminal wealth and the robustness of choosing suitable free parameters of $K$ and $L$. Furthermore, as the terminal wealth of the NN-based method with the order of Markov process $k=1$ was the highest among $k=\{1,2, \ldots, 5\}$ for all of $l=\{1,2, \ldots, 10\}$, we can infer that the market vector $\boldsymbol{x}_{n}$ is a first-order Markov process rather than multiple-order.

Horváth and Urbán (2012) defined the sets of possible portfolio vectors of OPS with short selling or leverage (i.e. borrowing money). In order to allow short selling, the original constraints of the proportion of a portfolio invested in asset $j, b^{(j)} \in[0,1]$ and $\sum_{j=1}^{d} b^{(j)}=1$, are replaced with $b^{(j)} \in[-1,1]$ and $\sum_{j=1}^{d} b^{(j)^{+}}=1$, where $x^{+} \stackrel{\text { def }}{=} \max (0, x)$. In order to allow borrowing money, the original constraints are replaced with $b^{(j)} \in[0, \infty)$ and $\sum_{j=1}^{d} b^{(j)}=B$, where $B$ is the maximum investable amount. The three sets (short selling only, leverage only, and both) are applicable to any OPS method without TCs.

\footnotetext{
$1 \arg \min _{\boldsymbol{b} \in \Delta^{d-1}}(\langle\boldsymbol{b}, \boldsymbol{\Sigma} \boldsymbol{b}\rangle-\langle\boldsymbol{b}, \boldsymbol{m}\rangle)$ in (7) is a convex optimisation problem (technically, a quadratic optimisation problem); thus, any local solution is guaranteed to be a global solution.
} 


\subsection{Online portfolio selection with transaction costs}

UP with TCs by Blum and Kalai (1999) is a trivial extension of UP (Cover 1991). Instead of accumulated wealth without TCs $S_{n}$ in (2), that with TCs $N_{n}$ (net wealth $N_{n}$ is defined in Section 5) is used to calculate the portfolio vector of the next period $n+1$ :

$$
\boldsymbol{b}_{n+1}=\frac{\int_{\Delta^{d-1}} \boldsymbol{b} N_{n}\left(\boldsymbol{b}, \boldsymbol{x}_{1: n}\right) f(\boldsymbol{b}) d \boldsymbol{b}}{\int_{\Delta^{d-1}} N_{n}\left(\boldsymbol{b}, \boldsymbol{x}_{1: n}\right) f(\boldsymbol{b}) d \boldsymbol{b}}
$$

where $N_{n}\left(\boldsymbol{b}, \boldsymbol{x}_{1: n}\right)=S_{0} \prod_{i=1}^{n}\left(\left\langle\boldsymbol{b}, \boldsymbol{x}_{i}\right\rangle-C_{i}\right)$ is wealth at the end of the $n$-th period with an initial wealth $S_{0}$ and the TC at the end of the $i$-th period $C_{i}$.

Györfi and Vajda (2008) i) extended the investment strategy by Györfi et al. (2006) by considering TCs and ii) simplified the assumption of the market from the multiple-order Markov process to a first-order Markov process. They suggested an implementable but suboptimal algorithm with one free parameter $L$ :

$$
\boldsymbol{b}_{n+1}=\frac{\sum_{l=1}^{L} \boldsymbol{h}^{(l)}\left(\boldsymbol{x}_{1: n}\right) S_{n}\left(\boldsymbol{h}^{(l)}\left(\boldsymbol{x}_{1: n}\right), \boldsymbol{x}_{1: n}\right)}{\sum_{l=1}^{L} S_{n}\left(\boldsymbol{h}^{(l)}\left(\boldsymbol{x}_{1: n}\right), \boldsymbol{x}_{1: n}\right)},
$$

where $S_{n}\left(\boldsymbol{h}^{(l)}\left(\boldsymbol{x}_{1: n}\right), \boldsymbol{x}_{1: n}\right)=S_{0} \prod_{i=1}^{n}\left\langle\boldsymbol{h}^{(l)}\left(\boldsymbol{x}_{1: n}\right), \boldsymbol{x}_{i}\right\rangle$ is wealth at the end of the $n$-th period with an initial wealth $S_{0} \cdot{ }^{1}$ The portfolio vector of the expert $\boldsymbol{h}^{(l)}(\cdot)$ from the series of past and current market vectors $\boldsymbol{x}_{1: n}$ is

$$
\boldsymbol{h}^{(l)}\left(\boldsymbol{x}_{1: n}\right)=\left\{\begin{array}{cl}
\boldsymbol{b}_{1}, & \text { if } J_{n}=\varnothing \\
\underset{\boldsymbol{b} \in \Delta^{d-1}}{\arg \max } \sum_{i \in J_{n}}\left(\ln \left\langle\boldsymbol{b}, \boldsymbol{x}_{i+1}\right\rangle+\ln w_{n}\right), & \text { otherwise }
\end{array}\right.
$$

where $J_{n}$ is the locations of matches between past market vector $\boldsymbol{x}_{i}$ and current one $\boldsymbol{x}_{n}$ :

$$
J_{n}=\left\{1 \leq i \leq n-1 \mid\left\|\boldsymbol{x}_{i}-\boldsymbol{x}_{n}\right\| \leq \sqrt{0.0001 d l}\right\} .
$$

I.e. the difference between (4) and (11) is only the addition of $\ln w_{n}$ (transaction cost factor $w_{n}$ will be explained in Section 5) that considers TCs. ${ }^{2}$

Kozat and Singer (2011) proposed a sequential universal portfolio whose achieved wealth is asymptotically as large as the wealth achieved by the best semi-constant rebalanced portfolio: a semi-constant rebalanced portfolio rebalances its portfolio only on selected instants to reduce TCs, while a constant rebalanced portfolio rebalances it at every period.

Bean and Singer (2012) combined UP with side information (Cover and Ordentlich 1996) and UP with TCs (Blum and Kalai 1999). Also, they employed factor graphs and a sum-product algorithm to derive computationally more efficient implementations of the combined UP. UP with side information under TCs (Bean and Singer 2012) achieves equal or greater wealth than UP with side information (Cover and Ordentlich 1996) under all simulated fixed percentage commissions. However, the Bean and Singer's method underperforms that of Blum and Kalai (1999).

\footnotetext{
1 Györfi and Vajda (2008) compared between i) aggregation with wealth (i.e. the expert in (11) makes the portfolio selection and pays TC individually) and ii) aggregation with portfolio (i.e. the aggregated portfolio $b_{n+1}$ in (10) pays TC), which implies that the former uses equations (11) and (12) while the latter uses equations (10), (11), and (12). Their numerical experiments showed that the latter outperformed the former.

2 Györfi and Vajda (2008) also introduced an optimal but unimplementable algorithm which solves a theoretical dynamic programming problem.
} 
Table 1. A 5-level limit order book of Microsoft Corporation, traded on NASDAQ, on 21 Jun 2012 at 16:00:00 (downloaded from https://lobsterdata.com/info/DataSamples.php). Bid-ask spread is USD 0.01, and midpoint price is USD 30.135 .

\begin{tabular}{rcrr}
\hline & Level & Price (USD) & Volume (shares) \\
\hline \multirow{4}{*}{ Asks } & 5 & 30.18 & 110,006 \\
& 4 & 30.17 & 86,886 \\
& 3 & 30.16 & 65,399 \\
& 2 & 30.15 & 80,663 \\
\hline \multirow{4}{*}{ Bids } & 1 & 30.14 & 16,600 \\
\hline & -1 & 30.13 & $-50,426$ \\
& -2 & 30.12 & $-83,306$ \\
& -3 & 30.11 & $-8,506$ \\
& -4 & 30.10 & $-43,838$ \\
& -5 & 30.09 & $-167,371$ \\
\hline
\end{tabular}

Györfi and Walk (2012) extended the theoretical and unimplementable dynamic programming algorithm developed by Györfi and Vajda (2008) to two data-driven algorithms of the log-optimal investment, based on a partitioning-based portfolio selection rule and $K$-nearest-neighbour-based rule. However, both are still unimplementable (i.e. the algorithms cannot be transformed into a computer program) for rebalancing a portfolio.

The numerical results of Ormos and Urbán (2013) show that the implementable log-optimal strategy (Györfi and Vajda 2008) generates greater alpha values (excess return) of the CAPM or Fama-French model than a buy-and-hold strategy, even in the presence of proportional TCs (TC rate was set to $0.1 \%$ both for sale and purchase). Therefore, the log-optimal portfolio strategy shows some kind of market inefficiency, in the sense that the first-order Markov model is better than random stock selection.

A threshold rebalanced portfolio trades stocks only if a fraction of the portfolio is below a lower threshold or over an upper threshold to reduce TCs (Tunc et al. 2013). However, this approach is only available in two-stock markets, although Tunc et al. mentioned the possibility of its extension to markets having more than two stocks. Furthermore, their assumption that the prices of the two stocks follow two independent geometric Brownian motions does not consider the correlation between the two stocks.

Das et al. (2013) assumed that the portfolio vector in the current period is replicated in the next period (i.e. $\boldsymbol{x}_{n}=\boldsymbol{x}_{n+1}$ ) and added the transaction penalty term $\alpha\left\|\boldsymbol{b}_{n+1}-\boldsymbol{b}_{n}\right\|_{1}$, where $\|\cdot\|_{1}$ denotes the $L^{1}$ norm. The disadvantage of this method is that the parameter $\alpha$ which controls the amount of trading should be properly chosen, whereas Györfi and Vajda (2008)'s method does not require the user's care. Besides, Das et al. (2014) added a group sparsity term to the Das et al. (2013)'s method to increase portfolio weights on a few top performing sectors and beat the market. However, the additional term is also controlled by a user parameter. Hence, the performance of this OPS algorithm depends on both the user parameters: the transaction penalty parameter and the group sparsity parameter.

\section{Market impact costs}

Market impact costs (MICs, also called price impact costs) can be generated by an investor who trades on an asset, pushing the price up when buying the asset and pushing it down while selling (Damodaran 2012, Chapter 5).

\subsection{Limit order book}

An LOB (Table 1 is an example) is defined as the current set of active limit orders that is sent to, and maintained by, a security exchange or a security dealer (Levy and Post 2005, p. 68). 


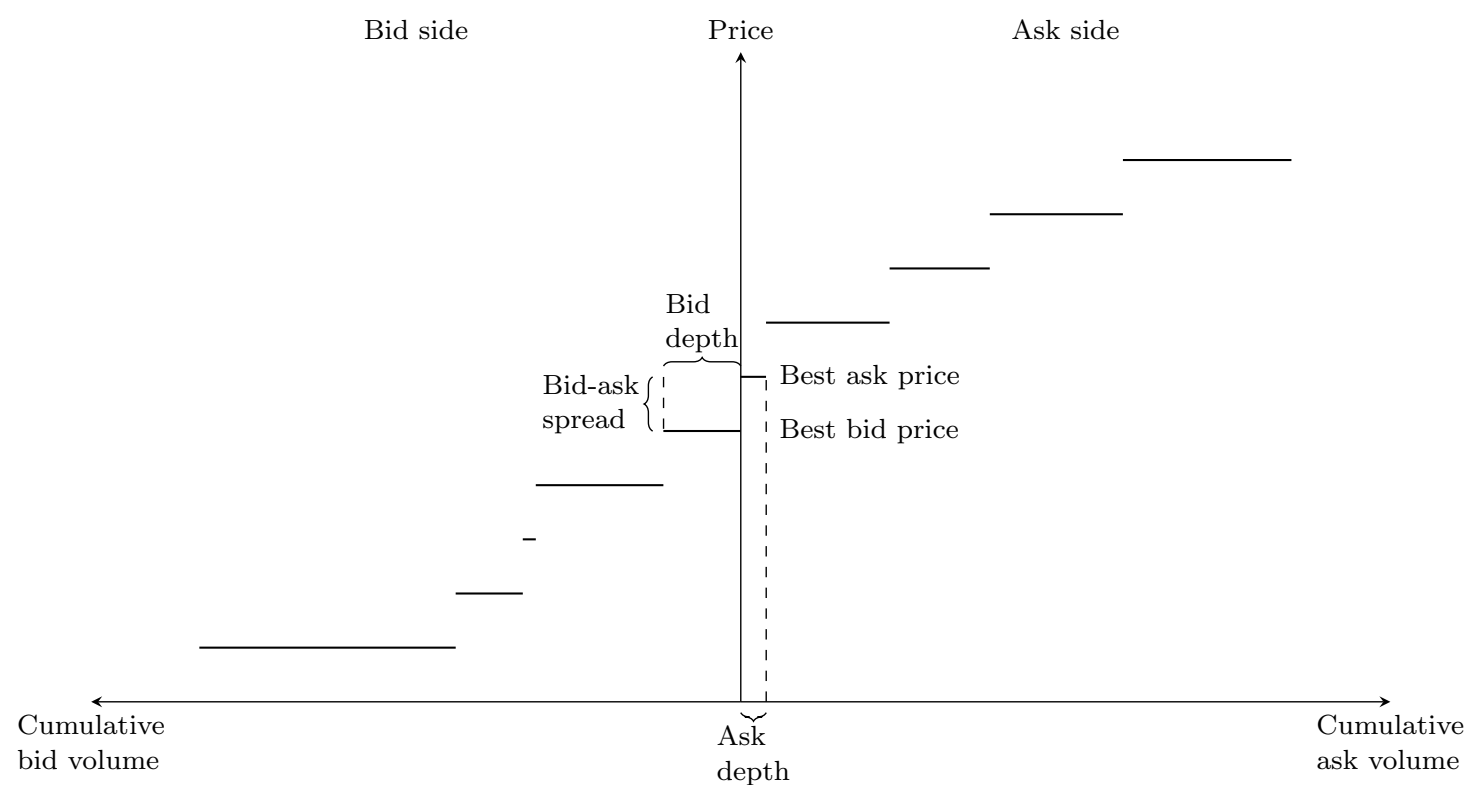

Figure 2. The liquidity of the limit order book in Table 1 (a similar diagram is in (Pristas 2007, p. 22)). The horizontal axis represents the cumulative volumes of the bid (ask) side on the left (right) hand side. The vertical axis shows the quoted prices.

\section{2. $\quad$ Market impact costs of online portfolio selection}

All the previous studies of OPS in Section 3 did not consider MICs but assumed that one can buy or sell any quantities of stock at its closing price. However, this is impracticable due to bid-ask spread and the finite depth of an LOB (Figure 2 shows both of them).

The bid-ask spread causes MICs. ${ }^{1}$ The closing price is the last price at which a stock is traded on a day, and it is either the lowest ask price (if a buyer buys) or the highest bid price (if a seller sells). Therefore, it is possible either i) that the purchase price is greater than the closing price or ii) that the sale price is less than the closing price, which in turn will make the terminal wealth of OPS less than the ideal case of zero bid-ask spread. In other words, the gap between the closing price and the purchase (or sale) price occurs in every period and generates TCs whenever OPS rebalances a portfolio.

Furthermore, if the ask or bid depth (see Figure 2) is shallow, MICs increase as the size (in dollars) of a portfolio increases. If an OPS algorithm intends to trade for the amount greater than the depth, the order will be executed for the amount of the depth at the best quoted price (i.e. the lowest ask price or the highest bid price), and then the remaining part of the order will be executed at the next prices of an LOB, which in turn will increase MICs and decrease the terminal wealth of OPS.

Consequently, LOBs (e.g. Table 1) as well as fixed percentage TCs (e.g. brokerage commissions and transaction taxes) should be considered to make OPS strategies practical.

\subsection{Market impact costs as a function of order size in a limit order book}

MICs that occur when rebalancing a portfolio can be written as a function of order volumes and prices in LOBs. The average MIC as a function of order size $q$ is defined as (Olsson 2005,

1 MICs include the bid-ask spread costs in this paper although they are separate in (Damodaran 2012). 


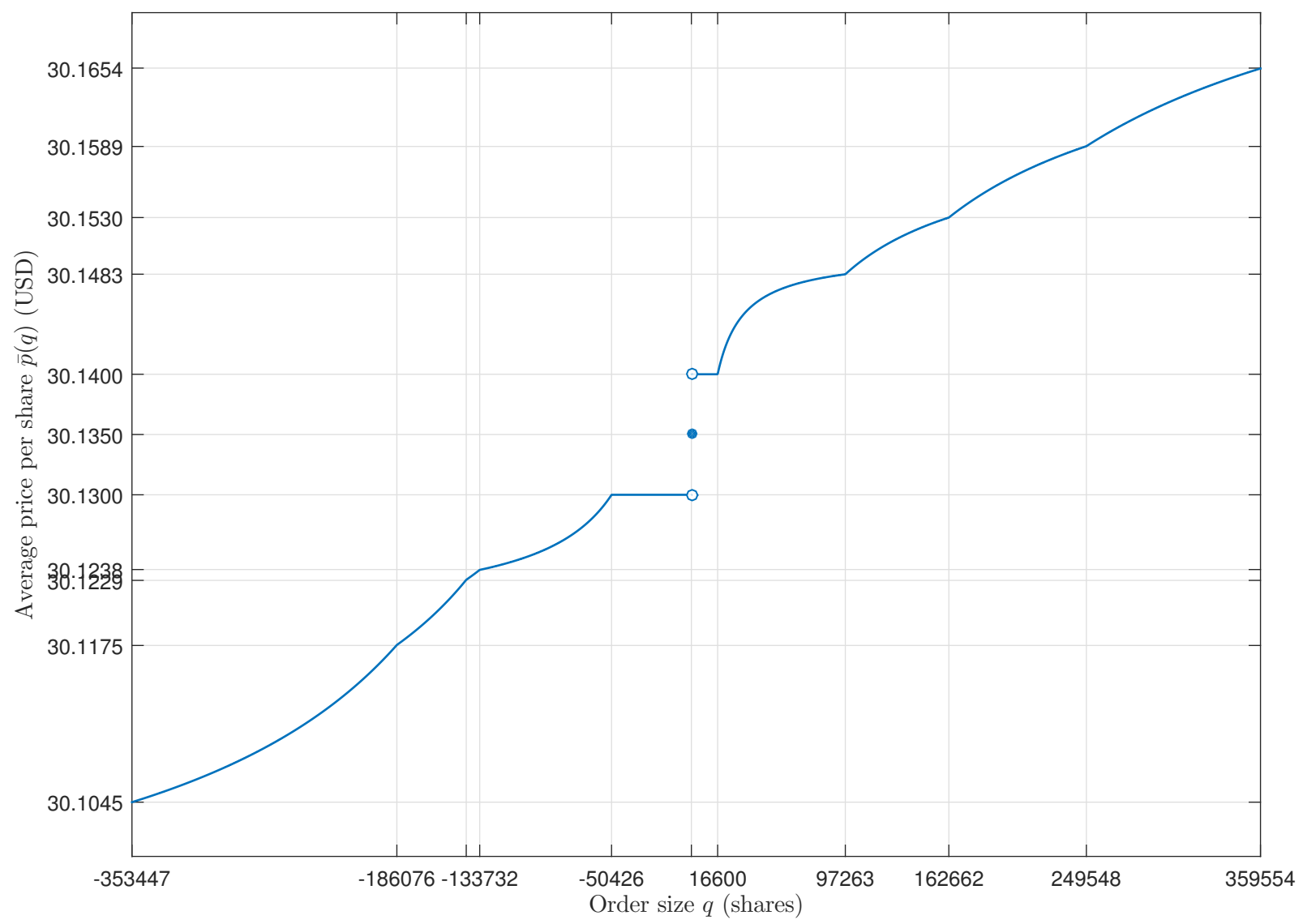

Figure 3. Average price per share $\bar{p}(q)$ for order size $q$ from the LOB data in Table 1. Positive (negative) $q$ means buying (selling) stocks.

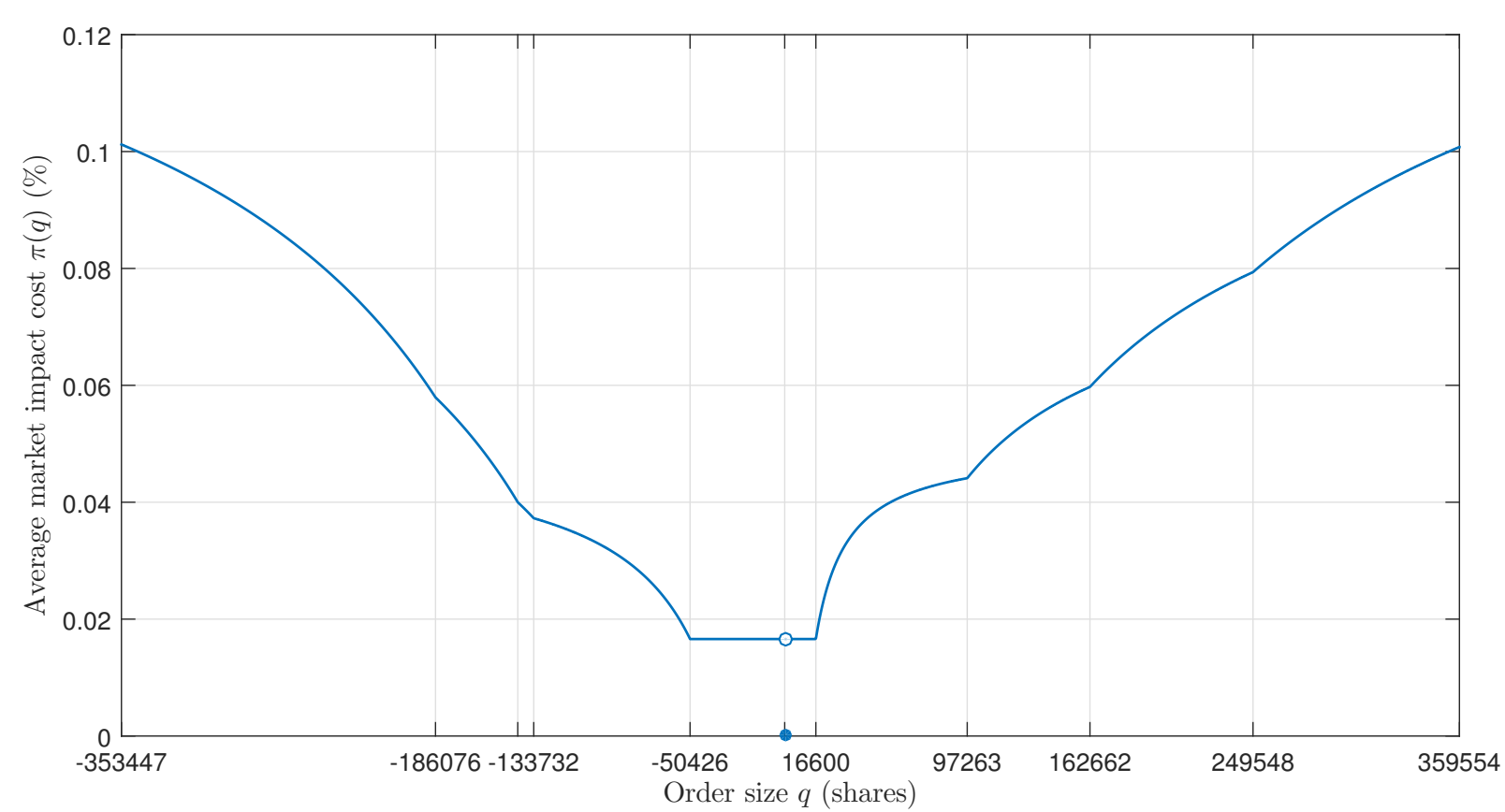

Figure 4. Average market impact cost $\pi(q)$ for order size $q$ from the LOB data in Table 1 . Positive (negative) $q$ means buying (selling) stocks. 
Chapter 2.3)

$$
\begin{gathered}
\pi\left(q, M, P_{1}, P_{2}, \ldots, P_{-1}, P_{-2}, \ldots, V_{1}, V_{2}, \ldots, V_{-1}, V_{-2}, \ldots\right) \\
\stackrel{\operatorname{def}}{=} \frac{\left|\bar{p}\left(q, M, P_{1}, P_{2}, \ldots, P_{-1}, P_{-2}, \ldots, V_{1}, V_{2}, \ldots, V_{-1}, V_{-2}, \ldots\right)-M\right|}{M},
\end{gathered}
$$

where $M=\frac{P_{-1}+P_{1}}{2}$ is the midpoint between the best bid and ask price, called mid price. The average price per share for the order size $q$ is defined as (Olsson 2005, Chapter 2.3)

$$
\begin{aligned}
& \bar{p}\left(q, M, P_{1}, P_{2}, \ldots, P_{-1}, P_{-2}, \ldots, V_{1}, V_{2}, \ldots, V_{-1}, V_{-2}, \ldots\right) \\
& \text { def }\left\{\begin{array}{cl}
\frac{\sum_{i=k+1}^{-1} P_{i} V_{i}+P_{k}\left(q-\sum_{i=k+1}^{-1} V_{i}\right)}{q}, & \text { if } q<V_{-1} \\
P_{-1}, & \text { if } V_{-1} \leq q<0 \\
M, & \text { if } q=0 \\
P_{1}, & \text { if } 0<q \leq V_{1} \\
\frac{\sum_{i=1}^{k-1} P_{i} V_{i}+P_{k}\left(q-\sum_{i=1}^{k-1} V_{i}\right)}{q}, & \text { if } V_{1}<q
\end{array},\right.
\end{aligned}
$$

where positive (negative) $q$ means buying (selling) stocks, $P_{i}$ and $V_{i}$ with positive (negative) $i$ are the quoted ask (bid) price and volume at level $i$, respectively (i.e. $P_{i}$ and $V_{i}$ correspond to the price and volume column of Table 1 , respectively, and $V_{i} \geq 0, V_{-i} \leq 0, \forall i \in \mathbb{Z}_{\geq 1}$ ), and the highest (lowest) trading level $k$ when $q>V_{1}\left(q<V_{-1}\right)$ is

$$
\begin{gathered}
k=\left\{x \in \mathbb{Z}_{\geq 2} \mid \sum_{i=1}^{x-1} V_{i}<q \leq \sum_{i=1}^{x} V_{i}\right\}, \\
\left(k=\left\{x \in \mathbb{Z}_{\leq-2} \mid \sum_{i=x}^{-1} V_{i} \leq q<\sum_{i=x+1}^{-1} V_{i}\right\}\right) .
\end{gathered}
$$

I.e. $k$ represents the level in the order book where the $q$-th share would be executed (Figure 3 and 4 show $\bar{p}(q)$ and $\pi(q)$ from the LOB data in Table 1$)$.

\section{Transaction cost factor}

The net wealth at the end of the $n$-th period $N_{n}$ is defined as (Györfi and Vajda 2008)

$$
N_{n} \stackrel{\text { def }}{=} S_{n}-C_{n}
$$

where $S_{n}$ is the gross wealth at the end of the $n$-th period, and $C_{n}$ is TC at the end of the $n$-th period. The current gross wealth $S_{n}$ can be calculated from the previous net wealth $N_{n-1}$ :

$$
S_{n}=N_{n-1} \sum_{j=1}^{d} b_{n}^{(j)} x_{n}^{(j)}=N_{n-1}\left\langle\boldsymbol{b}_{n}, \boldsymbol{x}_{n}\right\rangle
$$


where $x_{n}^{(j)}=\frac{s_{n}^{(j)}}{s_{n-1}^{(j)}}=\frac{M_{n}^{(j)}}{M_{n-1}^{(j)}}$ (i.e. the price of asset $j$ at the end of the $n$-th period $s_{n}^{(j)}$ is the mid price of asset $j$ at the end of the $n$-th period $\left.M_{n}^{(j)}\right)$.

The transaction cost factor (TCF) at the end of the $n$-th period (i.e. the present moment; see Figure 1) is defined as (Györfi and Vajda 2008)

$$
w_{n} \stackrel{\text { def }}{=} \frac{N_{n}}{S_{n}},
$$

where $w_{n} \in(0,1]$ and $w_{0} \stackrel{\text { def }}{=} 1$ (the subscript 0 denotes time 0 ; see Figure 1 ). Let us calculate $w_{n}$ when rebalancing from the current portfolio vector $\boldsymbol{b}_{n}$ to the next portfolio vector $\boldsymbol{b}_{n+1}$ (see Figure 1) for the following three cases: Section 5.1 describes an existing TCF model, while Section 5.2 and 5.3 describe a new TCF model proposed in this paper.

\subsection{Transaction cost factor with proportional costs but no market impact costs}

If there are no MICs, only mid prices are used to calculate TCF. Let $c_{p}$ and $c_{s}$ denote the rate of proportional TCs when purchasing and selling stocks, respectively, where $c_{p}, c_{s} \in[0,1): c_{p}+c_{s}>0$. At the present moment (see Figure 1), there are $b_{n}^{(j)} x_{n}^{(j)} N_{n-1}$ dollars of asset $j$ before rebalancing, by (17), while there are $b_{n+1}^{(j)} N_{n}$ dollars of asset $j$ after rebalancing. If $b_{n+1}^{(j)} N_{n}>b_{n}^{(j)} x_{n}^{(j)} N_{n-1}$, then we have to purchase asset $j$ for $b_{n+1}^{(j)} N_{n}-b_{n}^{(j)} x_{n}^{(j)} N_{n-1}$ dollars, and $c_{p}\left(b_{n+1}^{(j)} N_{n}-b_{n}^{(j)} x_{n}^{(j)} N_{n-1}\right)$ is the TC of asset $j$. On the other hand, if $b_{n}^{(j)} x_{n}^{(j)} N_{n-1}>b_{n+1}^{(j)} N_{n}$, then we have to sell asset $j$ for $b_{n}^{(j)} x_{n}^{(j)} N_{n-1}-b_{n+1}^{(j)} N_{n}$ dollars, and $c_{s}\left(b_{n}^{(j)} x_{n}^{(j)} N_{n-1}-b_{n+1}^{(j)} N_{n}\right)$ is TC of asset $j$.

The gross wealth $S_{n}$ consists of the sum of the net wealth $N_{n}$ and the TCs of all assets in the following self-financing way (Györfi and Vajda 2008):

$$
S_{n}=N_{n}+c_{p} \sum_{j=1}^{d}\left(b_{n+1}^{(j)} N_{n}-b_{n}^{(j)} x_{n}^{(j)} N_{n-1}\right)^{+}+c_{s} \sum_{j=1}^{d}\left(b_{n}^{(j)} x_{n}^{(j)} N_{n-1}-b_{n+1}^{(j)} N_{n}\right)^{+}
$$

where $x^{+} \stackrel{\text { def }}{=} \max (0, x)$. By dividing both sides of (19) by $S_{n}$ and by referring to (17) and (18), Equation (19) can be rewritten as (Györfi and Vajda 2008)

$$
1=w_{n}+c_{p} \sum_{j=1}^{d}\left(b_{n+1}^{(j)} w_{n}-\frac{b_{n}^{(j)} x_{n}^{(j)}}{\left\langle\boldsymbol{b}_{n}, \boldsymbol{x}_{n}\right\rangle}\right)^{+}+c_{s} \sum_{j=1}^{d}\left(\frac{b_{n}^{(j)} x_{n}^{(j)}}{\left\langle\boldsymbol{b}_{n}, \boldsymbol{x}_{n}\right\rangle}-b_{n+1}^{(j)} w_{n}\right)^{+} .
$$

Additionally, by using the property of $(a-b)^{+}=a-b+(b-a)^{+}$, Equation (20) can be simplified as (Ormos and Urbán 2013)

$$
w_{n}=1-\frac{c_{p}+c_{s}}{1+c_{p}} \sum_{j=1}^{d}\left(\frac{b_{n}^{(j)} x_{n}^{(j)}}{\left\langle\boldsymbol{b}_{n}, \boldsymbol{x}_{n}\right\rangle}-b_{n+1}^{(j)} w_{n}\right)^{+}
$$

which is solvable by using a root-finding algorithm, where $w_{n}=w\left(\boldsymbol{b}_{n}, \boldsymbol{b}_{n+1}, \boldsymbol{x}_{n}\right)$ is an unknown variable $\left(c_{p}\right.$ and $c_{s}$ are omitted for notational simplicity). ${ }^{1}$

1 Another form of TCF is defined as (Borodin and El-Yaniv 1998, pp. 299-300)

$$
w_{n}=1-\frac{c_{p}+c_{s}}{1+c_{p}} \sum_{j=1}^{d}\left(\frac{b_{n}^{(j)} x_{n}^{(j)}}{\left\langle\boldsymbol{b}_{n}, \boldsymbol{x}_{n}\right\rangle}-b_{n+1}^{(j)}\right)^{+}
$$



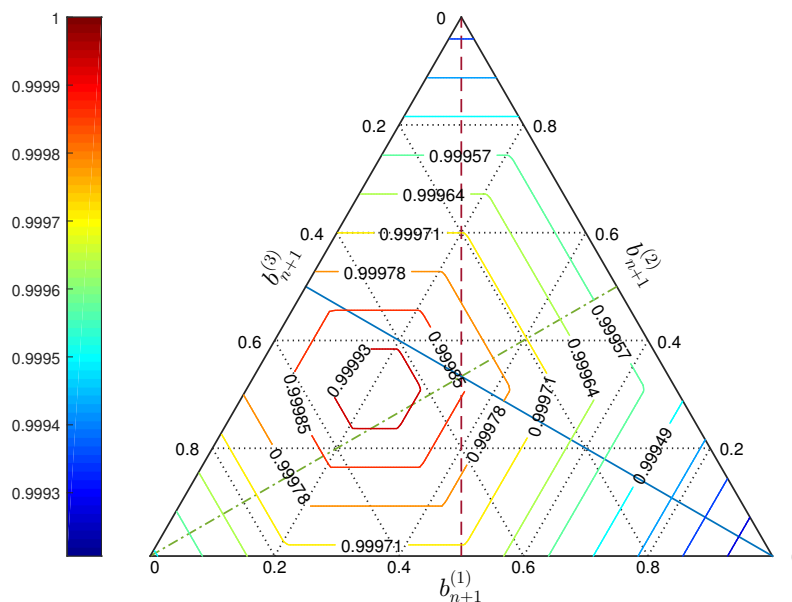

(a) Ternary contour plot of transaction cost factor when $c_{s}=0.1 \%$.
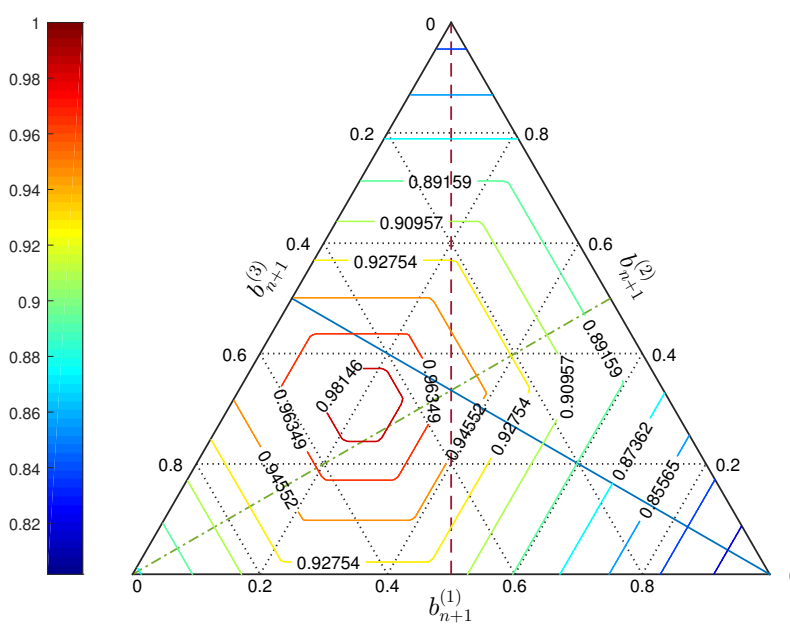

(c) Ternary contour plot of transaction cost factor when $c_{s}=25 \%$.
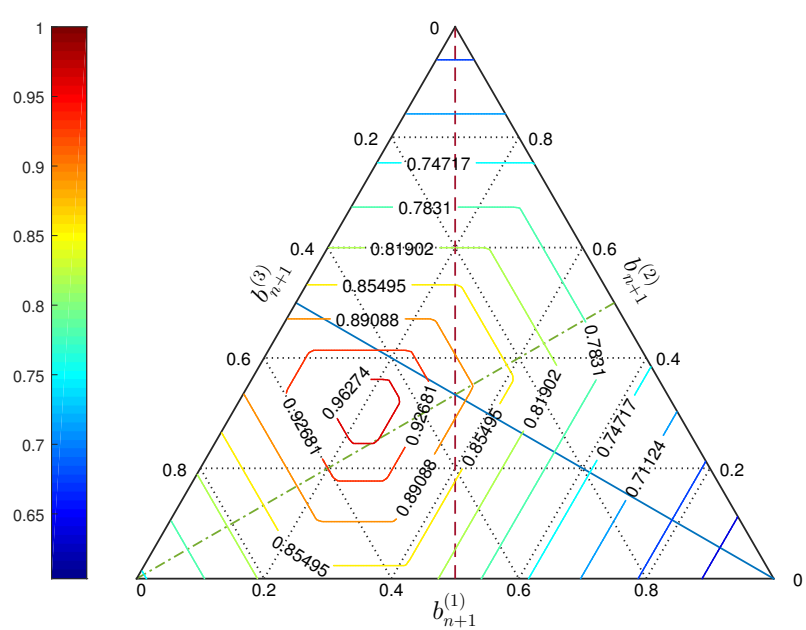

(e) Ternary contour plot of transaction cost factor when $c_{s}=50 \%$.

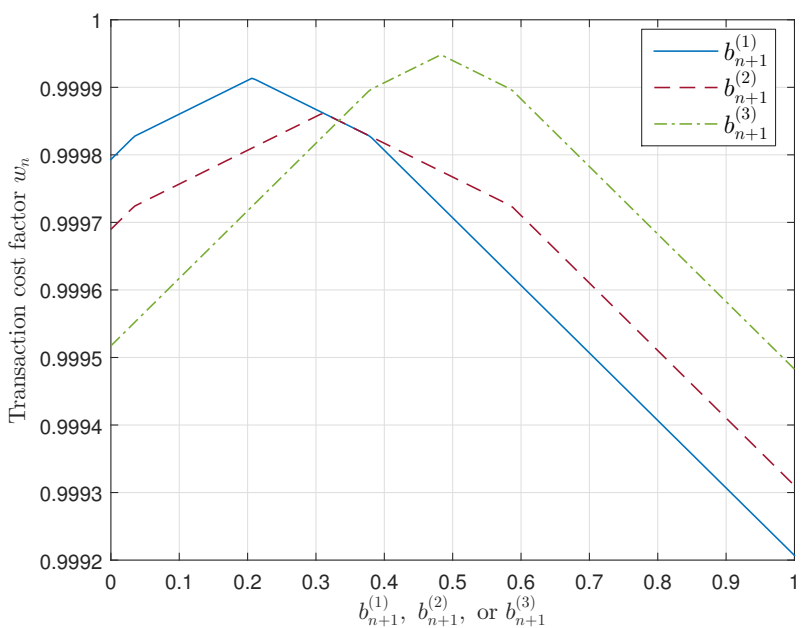

(b) $1 \mathrm{D}$ plots of transaction cost factor when $c_{s}=0.1 \%$ (each line matches each straight line in the left plot).

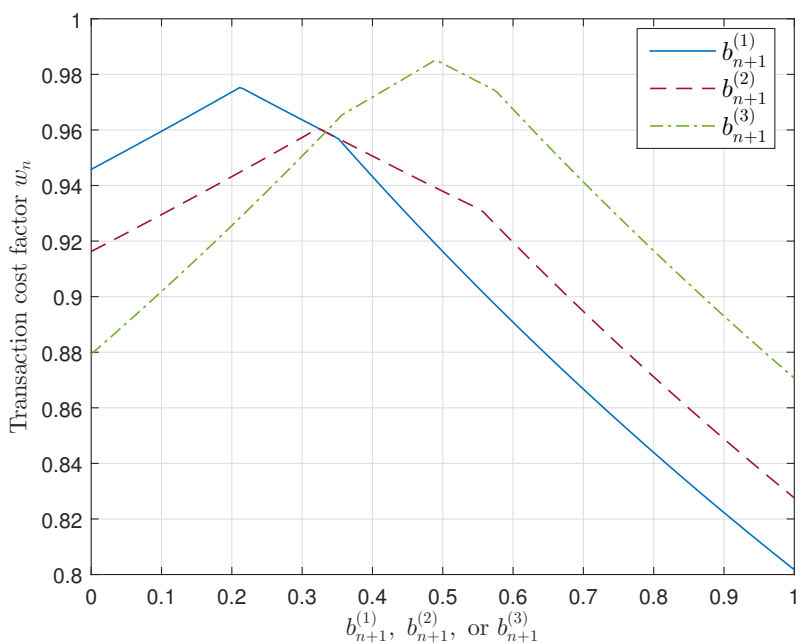

(d) $1 \mathrm{D}$ plots of transaction cost factor when $c_{s}=25 \%$ (each line matches each straight line in the left plot).

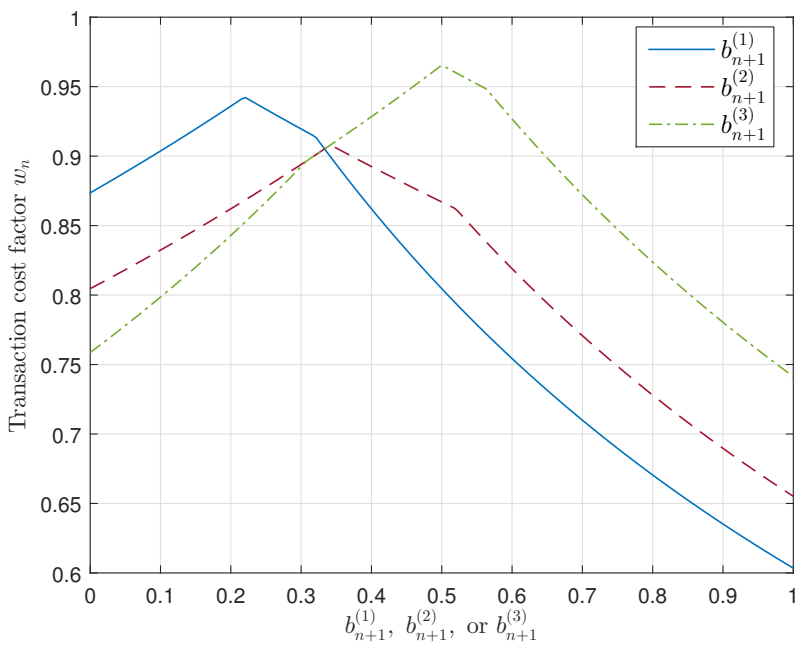

(f) $1 \mathrm{D}$ plots of transaction cost factor when $c_{s}=50 \%$ (each line matches each straight line in the left plot).

Figure 5. Ternary contour plots and $1 \mathrm{D}$ plots of transaction cost factor $w_{n}=w\left(\boldsymbol{b}_{n}, \boldsymbol{b}_{n+1}, \boldsymbol{x}_{n}\right)$ in (21) with the variable $\boldsymbol{b}_{n+1}$ and the fixed values: $\boldsymbol{b}_{n}=\left[\begin{array}{lll}1 / 3 & 1 / 3 & 1 / 3\end{array}\right]^{\mathrm{T}}, \boldsymbol{x}_{n}=\left[\begin{array}{lll}0.6 & 0.9 & 1.4\end{array}\right]^{\mathrm{T}}$, and $c_{p}=0$. 
TCF $w_{n}$ in (21) is a quasi-concave but not concave function of $\boldsymbol{b}_{n+1} \in \Delta^{d-1}$ (proof is in Appendix C) as shown in Figure 5(d) and 5(f), where its convexity depends on the TC rates, $c_{p}$ and $c_{s}$ : i.e. the greater $\frac{c_{p}+c_{s}}{1+c_{p}}$; the greater $\frac{\partial^{2} w_{n}}{\left(\partial b_{n+1}^{(j)}\right)^{2}}$, by (C8). Meanwhile, 1D plots in $5(\mathrm{~b})$ look like piecewise linear functions since $\frac{\partial^{2} w_{n}}{\left(\partial b_{n+1}^{(j)}\right)^{2}} \approx 0$ when $\frac{c_{p}+c_{s}}{1+c_{p}}$ is tiny.

LEMma 5.1 If $g: \mathbb{R}^{d} \rightarrow \mathbb{R}$ is quasi-concave (quasi-convex) and $h: \mathbb{R} \rightarrow \mathbb{R}$ is nondecreasing, then $f=h \circ g$ is quasi-concave (quasi-convex) (Boyd and Vandenberghe 2004, p. 102).

Lemma 5.2 The sum of quasi-concave (quasi-convex) functions is not necessarily quasi-concave (quasi-convex) (Sydsater et al. 2010, p. 95).

THEOREM $5.3 \quad \sum_{i \in J_{n}}\left(\ln \left\langle\boldsymbol{b}, \boldsymbol{x}_{i+1}\right\rangle+\ln w_{n}\right)$ in (11) is not necessarily a quasi-concave function of $\boldsymbol{b} \in \Delta^{d-1}$ even if $w_{n}$ is quasi-concave.

Proof. $\sum_{i \in J_{n}} \ln \left\langle\boldsymbol{b}, \boldsymbol{x}_{i+1}\right\rangle$ is quasi-concave both i) by the proof in Appendix B that $\sum_{i \in J_{n}} \ln \left\langle\boldsymbol{b}, \boldsymbol{x}_{i+1}\right\rangle$ is concave and ii) by the fact that every concave (convex) function is quasi-concave (quasi-convex). In addition, $\ln w_{n}$ is quasi-concave but not concave because $w_{n}$ is quasi-concave but not concave by lemma 5.1. As a result, $\sum_{i \in J_{n}}\left(\ln \left\langle\boldsymbol{b}, \boldsymbol{x}_{i+1}\right\rangle+\ln w_{n}\right)=\sum_{i \in J_{n}} \ln \left\langle\boldsymbol{b}, \boldsymbol{x}_{i+1}\right\rangle+\left|J_{n}\right| \ln w_{n}$ in (11) is not a necessarily quasi-concave function of $\boldsymbol{b} \in \Delta^{d-1}$ by lemma 5.2 .

Therefore, a local solution of (11) with TCF $w_{n}$ in (21) is not guaranteed to be a global solution. Györfi and Vajda (2008, p. 112) also mentioned that no global optimality of (11) is guaranteed although they did not give any proof.

\subsection{Transaction cost factor with market impact costs but no proportional costs}

This subsection is to propose a new TCF model by considering market impact costs but not considering proportional costs: if there are no proportional costs, the gross wealth $S_{n}$ consists of the sum of the net wealth $N_{n}$ and the MICs:

$$
S_{n}=N_{n}+\sum_{j=1}^{d}\left(\bar{p}\left(q_{n}^{(j)}\right)-M_{n}^{(j)}\right) q_{n}^{(j)},
$$

where $\bar{p}(\cdot)$ is the average price function in (14) (for notational simplicity, some input variables of (14), i.e. $M, P_{1}, P_{2}, \ldots, P_{-1}, P_{-2}, \ldots, V_{1}, V_{2}, \ldots, V_{-1}, V_{-2}, \ldots$ of each asset $j$, are omitted in (23) and the subsequent expressions), $M_{n}^{(j)}$ is the mid price of asset $j, q_{n}^{(j)}$ is an unknown order size of asset $j$, and subscript $n$ denotes the end of the $n$-th period.

When the investor either buys or sells every asset $j$ according to the portfolio vector of the $(n+1)$-th period $\boldsymbol{b}_{n+1}$, the following equation is satisfied:

$$
b_{n+1}^{(j)} N_{n}-b_{n}^{(j)} x_{n}^{(j)} N_{n-1}=M_{n}^{(j)} q_{n}^{(j)},
$$

where $b_{n}^{(j)} x_{n}^{(j)} N_{n-1}$ is the amount of dollars of asset $j$ before rebalancing a portfolio, and $b_{n+1}^{(j)} N_{n}$ is that after rebalancing. Hence, equation (24) means that the purchase (if left-hand side of (24) is positive) or sale (if left-hand side of (24) is negative) amount in dollars of asset $j$ equals the

which has less computational burden than (21), but it is error-prone if $\frac{c_{p}+c_{s}}{1+c_{p}}$ is large. Meanwhile, Borodin and El-Yaniv (1998, pp. 299-300) and Borodin et al. (2004, pp. 590-591) made the same typo of substituting $w_{n}=1-\frac{c_{p}+c_{s}}{1+c_{p}} \sum_{j=1}^{d}\left(\frac{b_{n}^{(j)} x_{n}^{(j)}}{\left\langle\boldsymbol{b}_{n}, \boldsymbol{x}_{n}\right\rangle}-b_{n}^{(j)}\right)^{+}$for $(22)$. 
product between $M_{n}^{(j)}$, the mid price of asset $j$, and $q_{n}^{(j)}$, an unknown order size of asset $j$, where positive (negative) $q_{n}^{(j)}$ means the purchase (sale) of asset $j .^{1}$

Equation (23) can be rewritten, by (18) and (24), as

$$
w_{n}=1-\frac{\sum_{j=1}^{d}\left(\bar{p}\left(q_{n}^{(j)}\right)-M_{n}^{(j)}\right) q_{n}^{(j)}}{S_{n}},
$$

where

$$
q_{n}^{(j)}=\frac{b_{n+1}^{(j)} S_{n} w_{n}-b_{n}^{(j)} x_{n}^{(j)} N_{n-1}}{M_{n}^{(j)}} .
$$

Equations (25) and (26) are solvable by using a root-finding algorithm, where $w_{n}=w\left(\boldsymbol{b}_{n}, \boldsymbol{b}_{n+1}, \boldsymbol{x}_{n}, N_{n-1}\right)$ is an unknown variable. TCF $w_{n}$ in (25) is calculated in the following order: i) $N_{0}=S_{0}$, ii) $S_{1}$ by using (17), iii) $w_{1}$ by using (25), iv) $N_{1}=w_{1} S_{1}$, v) $S_{2}$ by using (17), and vi) continued calculations.

TCF $w_{n}$ in (25) looks like a piecewise linear concave function as shown in Figure 6(b), 6(d), and $6(\mathrm{f}) .^{2}$ Also, the greater net wealth at the end of the previous period $N_{n-1}$; the less $w_{n}$ and more non-differentiable points in the 1D plots. This is because $|k|$ in (15), the absolute value of the executed level in LOB, increases as $N_{n-1}$ increases.

\subsection{Transaction cost factor with both proportional and market impact costs}

If both proportional TCs and MICs are considered, the gross wealth $S_{n}$ consists of the sum of the net wealth $N_{n}$, the MICs in (23), and the proportional TCs for buying and selling assets:

$$
S_{n}=N_{n}+\sum_{j=1}^{d}\left(\bar{p}\left(q_{n}^{(j)}\right)-M_{n}^{(j)}\right) q_{n}^{(j)}+c_{p} \sum_{j=1}^{d}\left(\bar{p}\left(q_{n}^{(j)}\right) q_{n}^{(j)}\right)^{+}+c_{s} \sum_{j=1}^{d}\left(-\bar{p}\left(q_{n}^{(j)}\right) q_{n}^{(j)}\right)^{+} 3^{3}
$$

This can be simplified, by the property of $a^{+}=a+(-a)^{+}$, as

$$
S_{n}=N_{n}+\sum_{j=1}^{d}\left(\left(1-c_{s}\right) \bar{p}\left(q_{n}^{(j)}\right)-M_{n}^{(j)}\right) q_{n}^{(j)}+\left(c_{p}+c_{s}\right) \sum_{j=1}^{d}\left(\bar{p}\left(q_{n}^{(j)}\right) q_{n}^{(j)}\right)^{+}
$$

and can be rewritten, by (18), as

$$
w_{n}=1-\frac{\sum_{j=1}^{d}\left(\left(1-c_{s}\right) \bar{p}\left(q_{n}^{(j)}\right)-M_{n}^{(j)}\right) q_{n}^{(j)}+\left(c_{p}+c_{s}\right) \sum_{j=1}^{d}\left(\bar{p}\left(q_{n}^{(j)}\right) q_{n}^{(j)}\right)^{+}}{S_{n}}
$$

1 The reason why $M_{n}^{(j)}$ in (24) cannot be replaced with $\bar{p}\left(q_{n}^{(j)}\right)$ is that $b_{n+1}^{(j)} N_{n}$ denotes the asset $j$ 's portion of the net wealth, excluding MICs, at the end of the $n$-th period. Also, $M_{n}^{(j)}$ excludes MIC, while $\bar{p}\left(q_{n}^{(j)}\right)$ includes MIC.

2 Mathematical proof of the convexity of $w_{n}$ in (25) is not given in this paper due to the complex equations: (14), (25), and (26). However, numerically approximate values of second-order partial derivative at differentiable points of the nine 1D plots in Figure 6(b), 6(d), and 6(f) are all positive when using the central difference $\frac{\partial^{2} f(x)}{\partial x^{2}} \approx \frac{f(x+h)-2 f(x)+f(x-h)}{h^{2}}$. This implies that $w_{n}$ in (25) is not concave.

3 The reason why the proportional TCs in (27) cannot be replaced with $c_{p} \sum_{j=1}^{d}\left(M_{n}^{(j)} q_{n}^{(j)}\right)^{+}+c_{s} \sum_{j=1}^{d}\left(-M_{n}^{(j)} q_{n}^{(j)}\right)^{+}$is that proportional TCs are determined by the actual traded price $\bar{p}\left(q_{n}^{(j)}\right)$, not by the mid price $M_{n}^{(j)}$. 


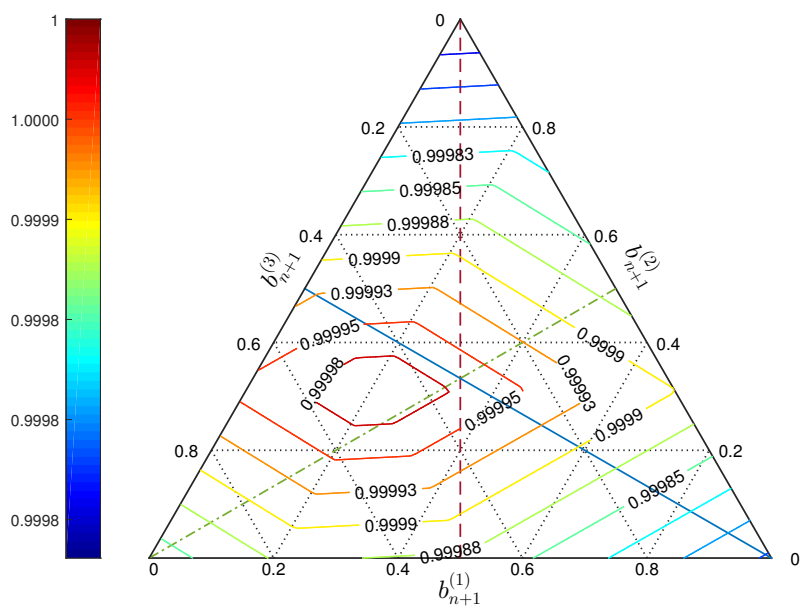

(a) Ternary contour plot of transaction cost factor when $N_{n-1}$ is USD $10^{4}$.
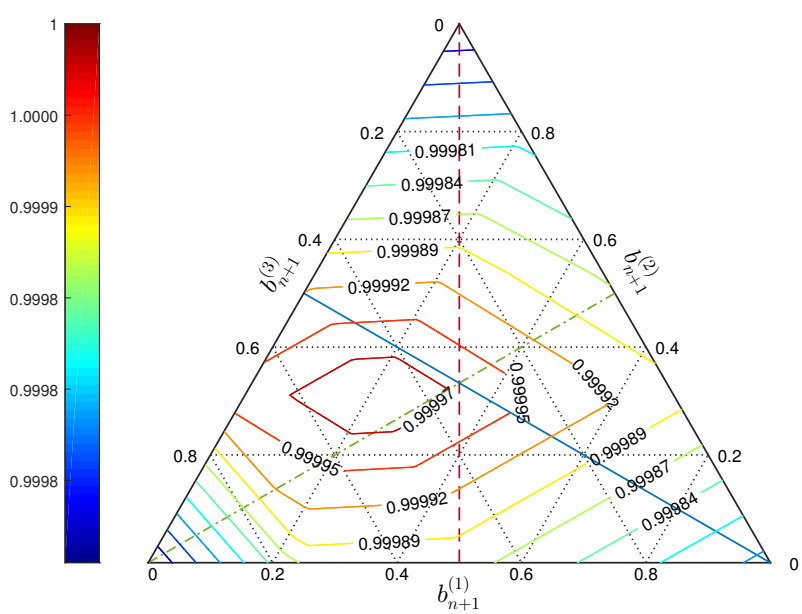

(c) Ternary contour plot of transaction cost factor when $N_{n-1}$ is $\operatorname{USD} 10^{5}$.
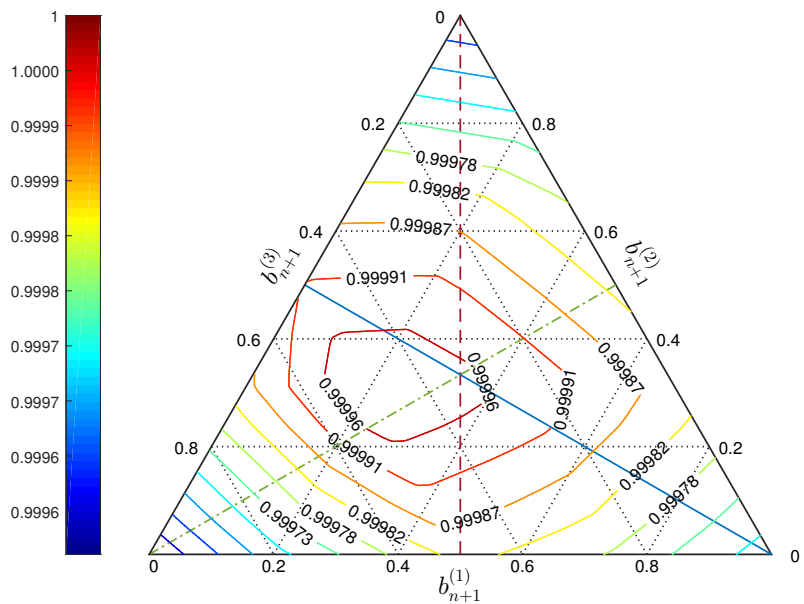

(e) Ternary contour plot of transaction cost factor when $N_{n-1}$ is USD $10^{6}$.

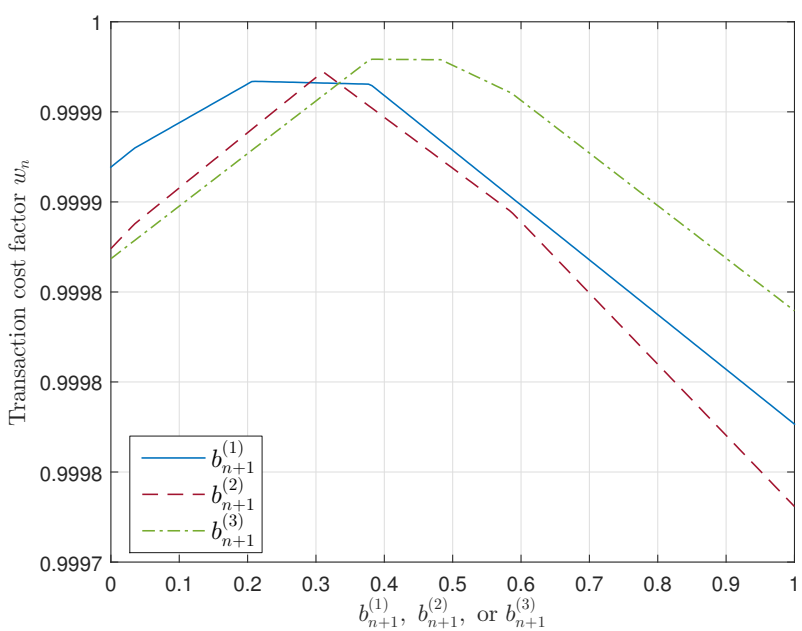

(b) $1 \mathrm{D}$ plots of transaction cost factor when $N_{n-1}$ is USD $10^{4}$ (each line matches each straight line in the left plot).

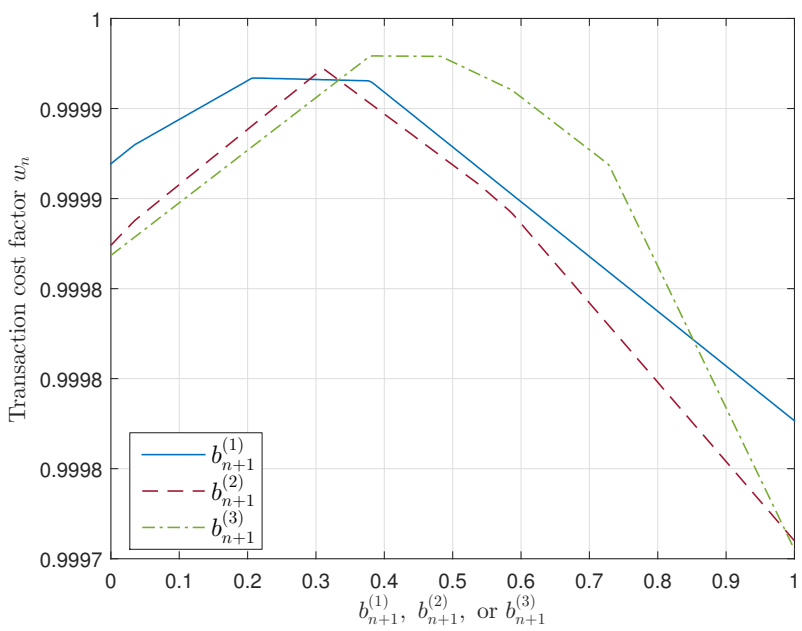

(d) 1 D plots of transaction cost factor when $N_{n-1}$ is USD $10^{5}$ (each line matches each straight line in the left plot).

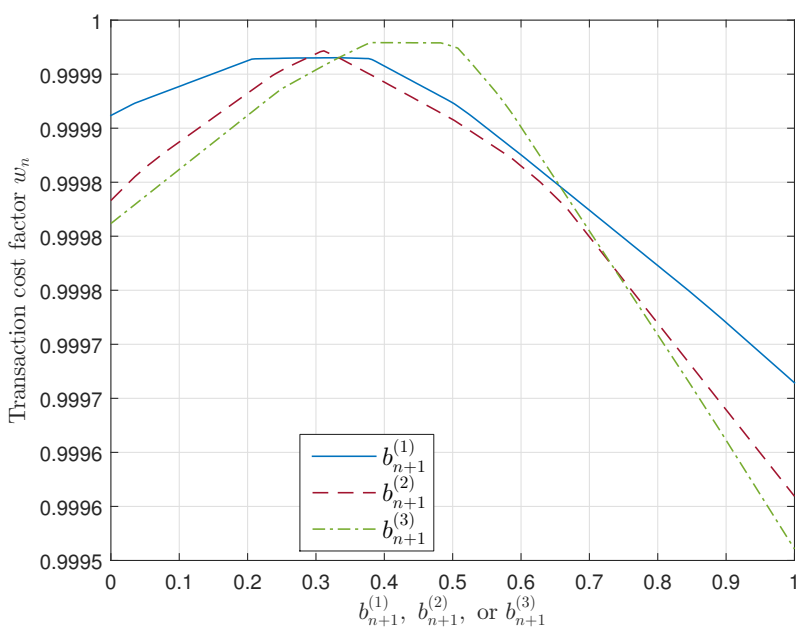

(f) $1 \mathrm{D}$ plots of transaction cost factor when $N_{n-1}$ is USD $10^{6}$ (each line matches each straight line in the left plot).

Figure 6. Ternary contour plots and 1D plots of transaction cost factor $w_{n}=w\left(\boldsymbol{b}_{n}, \boldsymbol{b}_{n+1}, \boldsymbol{x}_{n}, N_{n-1}\right)$ in (25) with the variable $\boldsymbol{b}_{n+1}$ and the fixed values: $\boldsymbol{b}_{n}=\left[\begin{array}{lll}1 / 3 & 1 / 3 & 1 / 3\end{array}\right]^{\mathrm{T}}$ and $\boldsymbol{x}_{n}=\left[\begin{array}{lll}0.6 & 0.9 & 1.4\end{array}\right]^{\mathrm{T}}$. 10-level limit order book data of AAPL $\left(b^{(1)}\right)$, AMZN $\left(b^{(2)}\right)$, and GOOG $\left(b^{(3)}\right)$ on 21 Jun 2012 at 16:00:00 was used. 
where $q_{n}^{(j)}$ is the same as (26). Equation (29) and (26) are also solvable by using a root-finding algorithm, where $w_{n}=w\left(\boldsymbol{b}_{n}, \boldsymbol{b}_{n+1}, \boldsymbol{x}_{n}, N_{n-1}\right)$ is an unknown variable $\left(c_{p}\right.$ and $c_{s}$ are omitted for notational simplicity).

The ternary contour plots and 1D plots of $w_{n}$ in (29) are different from those of $w_{n}$ in (21) when comparing between Figure $\{7(\mathrm{a}), 7(\mathrm{~b})\}$ and Figure $\{5(\mathrm{a}), 5(\mathrm{~b})\}$ due to the additional consideration of MICs. In contrast, they are similar when comparing between Figure $\{7(\mathrm{c}), 7(\mathrm{~d}), 7(\mathrm{e}), 7(\mathrm{f})\}$ and Figure $\{5(\mathrm{c}), 5(\mathrm{~d}), 5(\mathrm{e}), 5(\mathrm{f})\}$ because proportional TCs are much greater than MICs when $c_{p}$ or $c_{s}$ is large.

THEOREM 5.4 The quasi-concavity of function $f: \mathbb{R}^{n} \rightarrow \mathbb{R}$ is equivalent to the fact that $f$ is unimodal (i.e. single-peaked) (Simchi-Levi et al. 2014, p. 18).

TCF $w_{n}$ in (29) is a unimodal function of $\boldsymbol{b}_{n+1}$ (TCF $w_{n}$ in (21) and that in (25) are also unimodal), as shown in Figure 7(a), 7(c), and 7(e), because $w_{n}$ strictly decreases as $\boldsymbol{b}_{n+1}$ goes away from the maximum point:

$$
\boldsymbol{b}_{n+1}^{\star} \stackrel{\text { def }}{=} \underset{\boldsymbol{b} \in \Delta^{d-1}}{\arg \max } w\left(\boldsymbol{b}_{n}, \boldsymbol{b}, \boldsymbol{x}_{n}, N_{n-1}\right)=\frac{\boldsymbol{b}_{n} \odot \boldsymbol{x}_{n}}{\left\langle\boldsymbol{b}_{n}, \boldsymbol{x}_{n}\right\rangle},
$$

where $\odot$ denotes element-wise multiplication of vectors. ${ }^{1}$ Hence, $w_{n}$ in (29) is quasi-concave by theorem $5.4,{ }^{2}$ but $\sum_{i \in J_{n}}\left(\ln \left\langle\boldsymbol{b}, \boldsymbol{x}_{i+1}\right\rangle+\ln w_{n}\right)$ in (11) with $w_{n}$ in (29) is not a necessarily quasi-concave function of $\boldsymbol{b} \in \Delta^{d-1}$ by theorem 5.3.

\section{Log-optimal portfolio}

The log-optimal portfolio (or the log-utility approach) is one of OPS strategies and is the basis of the proposed method described in Section 7.

\subsection{Objective of log-optimal investment strategy ${ }^{3}$}

Assume that the present moment is time 0 (do not see Figure 1). The wealth of a portfolio of a single asset at the end of the $n$-th period (in the future) with an initial wealth $S_{0}$ is

$$
S_{n}=S_{0} \prod_{i=1}^{n} X_{i}
$$

where $X_{i}$ is a random variable of the price relative at the end of the $i$-th period (i.e. $X_{i}=\frac{S_{i}}{S_{i-1}}$, where $S_{i}$ is a random variable of an asset's price at the end of the $i$-th period). Taking the log of both the sides gives

$$
\ln S_{n}=\ln S_{0}+\sum_{i=1}^{n} \ln X_{i}
$$

\footnotetext{
1 The mathematical proof of the unimodality is not provided in this paper.

2 Mathematical proof of the convexity of $w_{n}$ in (29) is not given in this paper due to the complex equations: (14), (15), (29), and (26). However, numerically approximate values of second-order partial derivative at differentiable points of the nine 1D plots in Figure $7(\mathrm{~b}), 7(\mathrm{~d})$, and $7(\mathrm{f})$ are all positive when using the central difference $\frac{\partial^{2} f(x)}{\partial x^{2}} \approx \frac{f(x+h)-2 f(x)+f(x-h)}{h^{2}}$. This implies that $w_{n}$ in (29) is not concave.

3 The whole of this subsection is a paraphrase of (Luenberger 1998, pp. 419-421).
} 

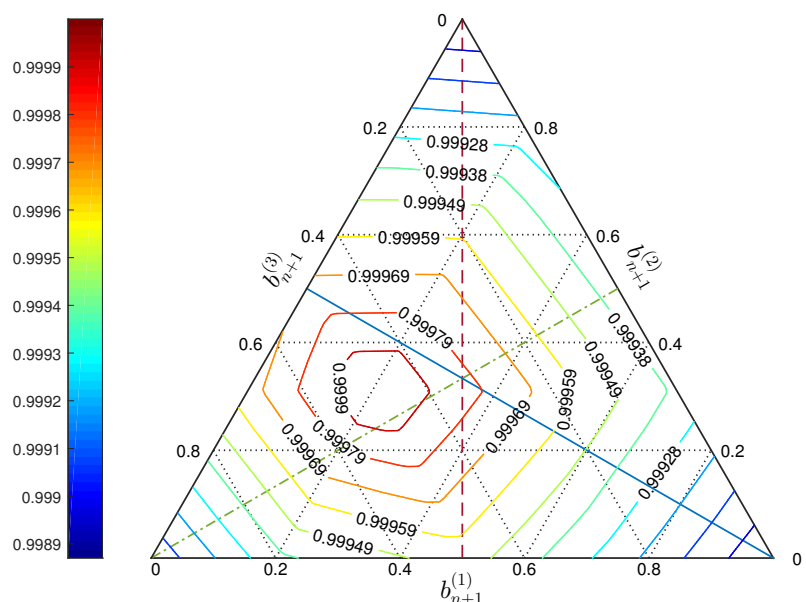

(a) Ternary contour plot of transaction cost factor when $c_{s}=0.1 \%$.
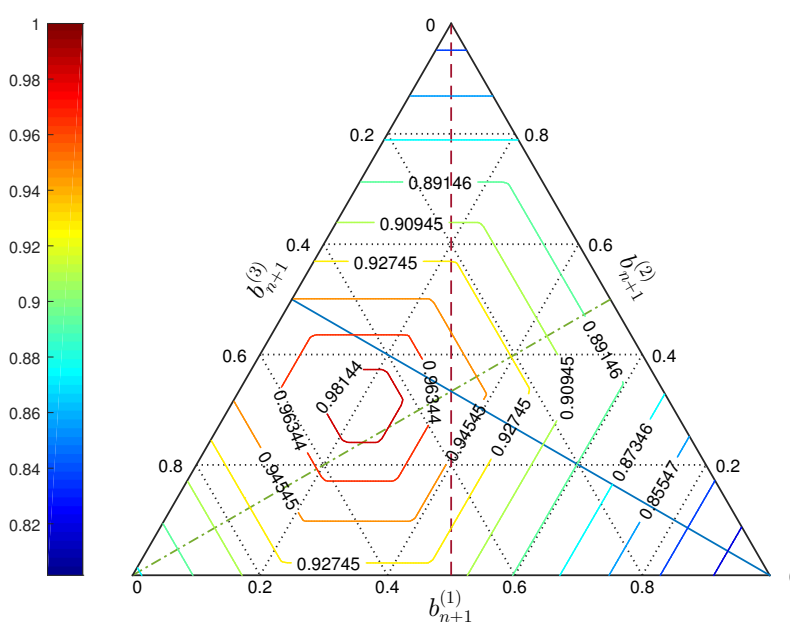

(c) Ternary contour plot of transaction cost factor when $c_{s}=25 \%$.
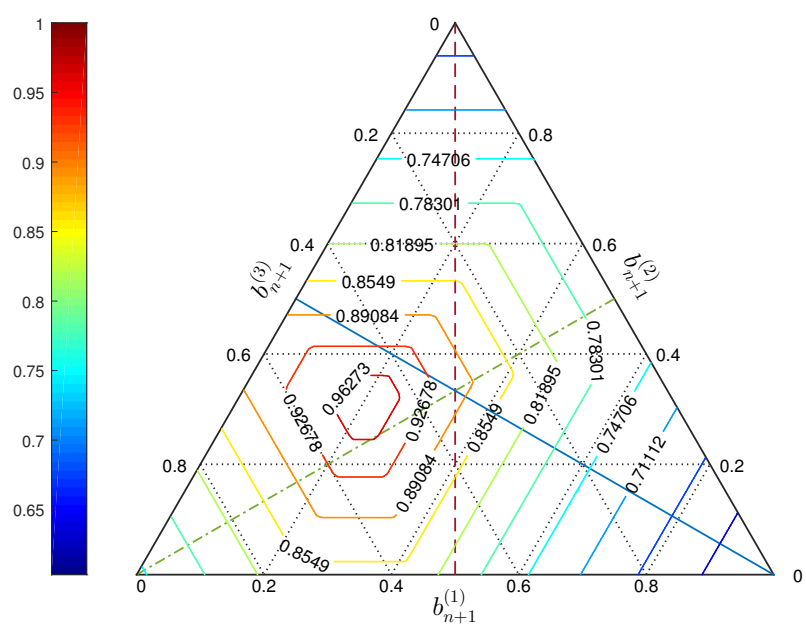

(e) Ternary contour plot of transaction cost factor when $c_{s}=50 \%$.

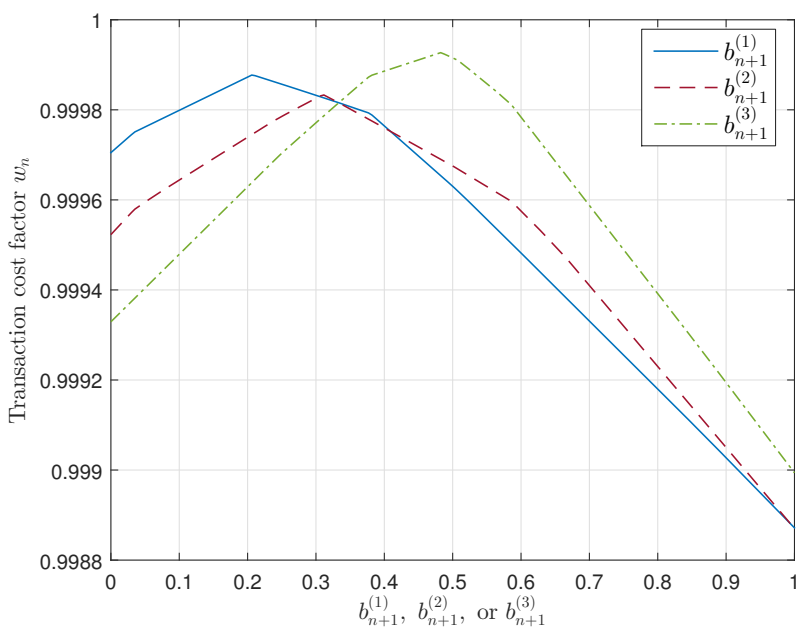

(b) $1 \mathrm{D}$ plots of transaction cost factor when $c_{s}=0.1 \%$ (each line matches each straight line in the left plot).

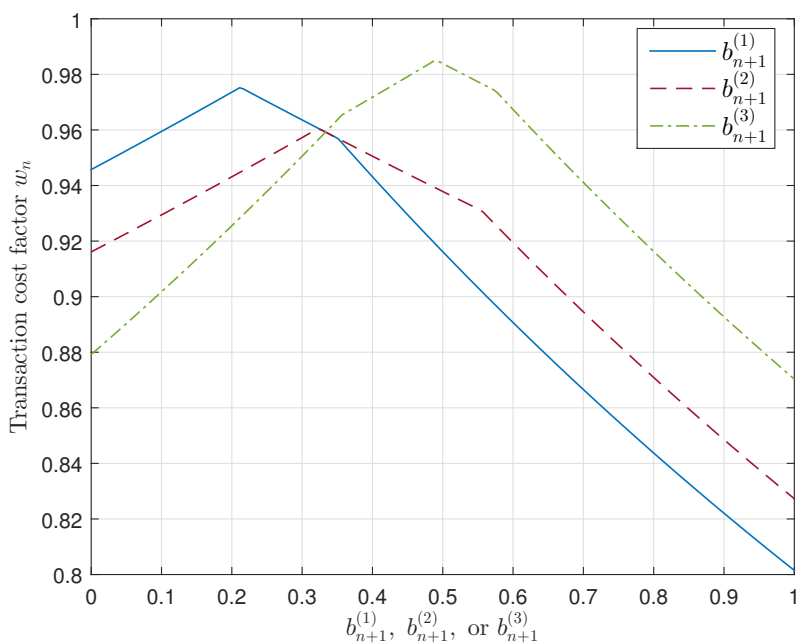

(d) $1 \mathrm{D}$ plots of transaction cost factor when $c_{s}=25 \%$ (each line matches each straight line in the left plot).

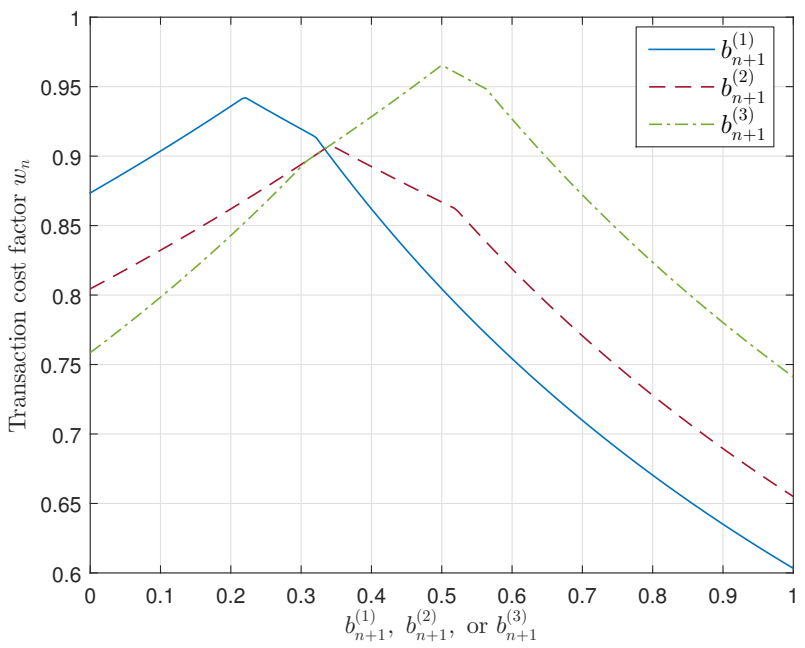

(f) $1 \mathrm{D}$ plots of transaction cost factor when $c_{s}=50 \%$ (each line matches each straight line in the left plot).

Figure 7. Ternary contour plots and 1D plots of transaction cost factor $w_{n}=w\left(\boldsymbol{b}_{n}, \boldsymbol{b}_{n+1}, \boldsymbol{x}_{n}, N_{n-1}\right)$ in (29) with the variable $\boldsymbol{b}_{n+1}$ and the fixed values: $\boldsymbol{b}_{n}=\left[\begin{array}{lll}1 / 3 & 1 / 3 & 1 / 3\end{array}\right]^{\mathrm{T}}, \boldsymbol{x}_{n}=\left[\begin{array}{lll}0.6 & 0.9 & 1.4\end{array}\right]^{\mathrm{T}}, c_{p}=0$, and $N_{n-1}$ is USD $10^{6}$. 10-level limit order book data of AAPL $\left(b^{(1)}\right)$, AMZN $\left(b^{(2)}\right)$, and GOOG $\left(b^{(3)}\right)$ on 21 Jun 2012 at 16:00:00 was used. 
and this can be rewritten as

$$
\ln \left(\frac{S_{n}}{S_{0}}\right)^{1 / n}=\frac{1}{n} \sum_{i=1}^{n} \ln X_{i}
$$

As $n$ becomes extremely large, the right-hand side of (33) converges to

$$
\lim _{n \rightarrow \infty} \frac{1}{n} \sum_{i=1}^{n} \ln X_{i}=\mathbb{E}\left[\ln X_{i}\right]
$$

under the assumption that the random variable $X_{i}$ is independent and identically distributed, and the wealth $S_{n}$ converges to

$$
\lim _{n \rightarrow \infty} S_{n}=S_{0} e^{m n}
$$

where $m \stackrel{\text { def }}{=} \mathbb{E}\left[\ln X_{i}\right]$ is a growth rate. Consequently, for large $n$ (i.e. long-term investment), the wealth $S_{n}$ grows (roughly) exponentially with $m n$; therefore, maximising the growth rate $m$ is critical for the long-term investment. In other words, the $\log$ is an appropriate utility function for the long-term investment.

\section{2. log-optimal portfolio with transaction costs}

The gross wealth at the end of the next period (i.e. $n+1$; see Figure 1), from (17) and (18), is

$$
S_{n+1}=w_{n} S_{n}\left\langle\boldsymbol{b}_{n+1}, \boldsymbol{X}_{n+1}\right\rangle,
$$

where $w_{n}=w\left(\boldsymbol{b}_{n}, \boldsymbol{b}_{n+1}, \boldsymbol{x}_{n}\right)$ is TCF in (21), the deterministic value $S_{n}$ is the gross wealth at the present moment, the $d$-dimensional variable $\boldsymbol{b}_{n+1}$ is the portfolio vector of the next period, and the $d$-dimensional multivariate random variable $\boldsymbol{X}_{n+1}$ is a set of possible market vectors in the next period.

Our aim is to choose an appropriate portfolio vector $\boldsymbol{b}_{n+1}$ to maximise the conditional expected value of the $\log$ (the $\log$ behaves as a utility function for the long-term investment as mentioned in Section 6.1) of the gross wealth at the end of the next period, given the observed market vectors $\boldsymbol{x}_{n}, \boldsymbol{x}_{n-1}, \ldots, \boldsymbol{x}_{1}$ :

$$
\begin{aligned}
\boldsymbol{b}_{n+1} & =\underset{\boldsymbol{b} \in \Delta^{d-1}}{\arg \max } \mathbb{E}\left[\ln S_{n+1}\right] \\
& =\underset{\boldsymbol{b} \in \Delta^{d-1}}{\arg \max } \mathbb{E}\left[\ln \left(w\left(\boldsymbol{b}_{n}, \boldsymbol{b}, \boldsymbol{x}_{n}\right) S_{n}\left\langle\boldsymbol{b}, \boldsymbol{X}_{n+1}\right\rangle\right)\right] \\
& =\underset{\boldsymbol{b} \in \Delta^{d-1}}{\arg \max }\left(\ln w\left(\boldsymbol{b}_{n}, \boldsymbol{b}, \boldsymbol{x}_{n}\right)+\mathbb{E}\left[\ln \left\langle\boldsymbol{b}, \boldsymbol{X}_{n+1}\right\rangle \mid \boldsymbol{X}_{n}=\boldsymbol{x}_{n}, \boldsymbol{X}_{n-1}=\boldsymbol{x}_{n-1}, \ldots, \boldsymbol{X}_{1}=\boldsymbol{x}_{1}\right]\right),
\end{aligned}
$$

and this can be rewritten as

$$
\boldsymbol{b}_{n+1}=\underset{\boldsymbol{b} \in \Delta^{d-1}}{\arg \max }\left(\ln w\left(\boldsymbol{b}_{n}, \boldsymbol{b}, \boldsymbol{x}_{n}\right)+\mathbb{E}\left[\ln \left\langle\boldsymbol{b}, \boldsymbol{X}_{n+1}\right\rangle \mid \boldsymbol{X}_{n}=\boldsymbol{x}_{n}\right]\right),
$$

under the assumption that the market process $\left\{\boldsymbol{X}_{i}\right\}$ is a homogeneous first-order Markov process. This result is the same as (Györfi and Vajda 2008, equation (5)) although they derived (38) in a different way. Finally, the implementable algorithm to obtain $\boldsymbol{b}_{n+1}$ in (38) employs Equation (11) and (12). 


\section{Proposed method of online portfolio selection}

\subsection{Assumptions for simplicity}

First, we assume i) that assets are arbitrarily divisible (i.e. $q_{n}^{(j)} \in \mathbb{R}, \forall j, n \geq 1$ ) to avoid mixed-integer nonlinear programming ${ }^{1}$ and ii) that the market impact of trading by the proposed OPS method is transitory, not persistent: i.e. portfolio rebalancing at the present moment does not affect the price at the end of the next period $s_{n+1}$ (see Figure 1$)^{2}$

Only market orders, an order to buy or sell a specific number of shares $q$ at the best price available when an investor places his or her order, are submitted when the proposed strategy rebalances a portfolio in order to avoid the risk of non-execution. In addition, the proposed method does not split a large market order into smaller market orders in order to avoid potential liquidity risk in the future: ${ }^{3}$ i.e. it rebalances a portfolio by using current LOBs (which are obvious) rather than by using LOBs in the future (which are unknown).

The proposed method ignores hidden limit orders (HLOs), invisible in limit order books. If a market order is executed against a hidden order, the trader submitting the market order may receive an unexpected price improvement (Bauwens et al. 2007, p. 115): i.e. if HLOs are in a limit order book, a trader may buy stocks at a cheaper price than expected, and he or she may sell stocks at a superior price than expected. In other words, $\tilde{\pi}(q) \leq \pi(q), \forall q \in \mathbb{R}$, where $\tilde{\pi}(q)$ is the average market impact cost with HLOs for order size $q$, and $\pi(q)$ is that without HLOs in (13). The additional liquidity from HLOs can be quantified by hidden volume rate, the total volume of trades against hidden orders divided by the total volume of all trades. The mean of this value between 2 Jan 2014 and 31 Dec 2015 is $\{13.30 \%, 13.33 \%\}$ in the case of stocks, not exchange traded products, traded on $\left\{\right.$ NYSE, NASDAQ\}, respectively. ${ }^{4}$ Therefore, the difference between $\tilde{\pi}(q)$ and $\pi(q)$ may not be significant.

Computation time to calculate $\boldsymbol{b}_{n+1}$ between receiving real-time data of LOBs from a stock exchange and sending market orders to rebalance a portfolio is ignored. To be specific, the price and volume in LOBs change during the computation as LOBs are continuously updated by other investors. Therefore, the proposed method actually uses the past LOBs, not the present, to calculate $\boldsymbol{b}_{n+1}$. In other words, it employs TCF $w_{n}$ in (29) delayed for the computation time.

\subsection{Details of the proposed method}

The proposed method is based on the log-optimal portfolio with TCs in (38), as the same as (Györfi and Vajda 2008). The difference between the existing (Györfi and Vajda 2008) and proposed method is $w_{n}$. I.e. $w_{n}=w\left(\boldsymbol{b}_{n}, \boldsymbol{b}_{n+1}, \boldsymbol{x}_{n}, N_{n-1}\right)$ in (29) is employed for the proposed method, whereas $w_{n}=w\left(\boldsymbol{b}_{n}, \boldsymbol{b}_{n+1}, \boldsymbol{x}_{n}\right)$ in (21) is employed for the existing method. As a result, the portfolio vector of the next period $\boldsymbol{b}_{n+1}$ of the proposed method depends on the net wealth of the previous period $N_{n-1}$, while that of the existing method does not (the proposed method has less tendency of portfolio rebalancing as $N_{n-1}$ increases, while the existing method does not).

Consequently, the proposed method uses the following equations: (10), (11), (12), (14), (15), (26), and (29), where (11) is a constrained (i.e. $\boldsymbol{b}_{n+1} \in \Delta^{d-1}$ ) nonlinear optimisation problem (Algorithm 1 describes the difference between the existing and proposed method). However, Equation (11) is not a quasi-convex optimisation problem for both the cases, (21) and (29), which means a local solution is not guaranteed to be a global solution, as mentioned in Section 5.1 and 5.3.

1 Softwares of mixed-integer nonlinear programming are listed in (Bussieck and Vigerske 2011).

2 Dynamic trading strategy by Gârleanu and Pedersen (2013) allows for both the cases: transitory and persistent market impact.

3 Expected overall market impact costs can be minimized by the split of a large market order into a number of smaller consecutive market orders under a specified resilience rate (Alfonsi et al. 2008).

4 This value is the average of daily hidden volume rates, downloaded from the homepage of U.S. Securities and Exchange Commission (URL: http://www.sec.gov/opa/data/market-structure/marketstructuredata-by-exchange.html). 


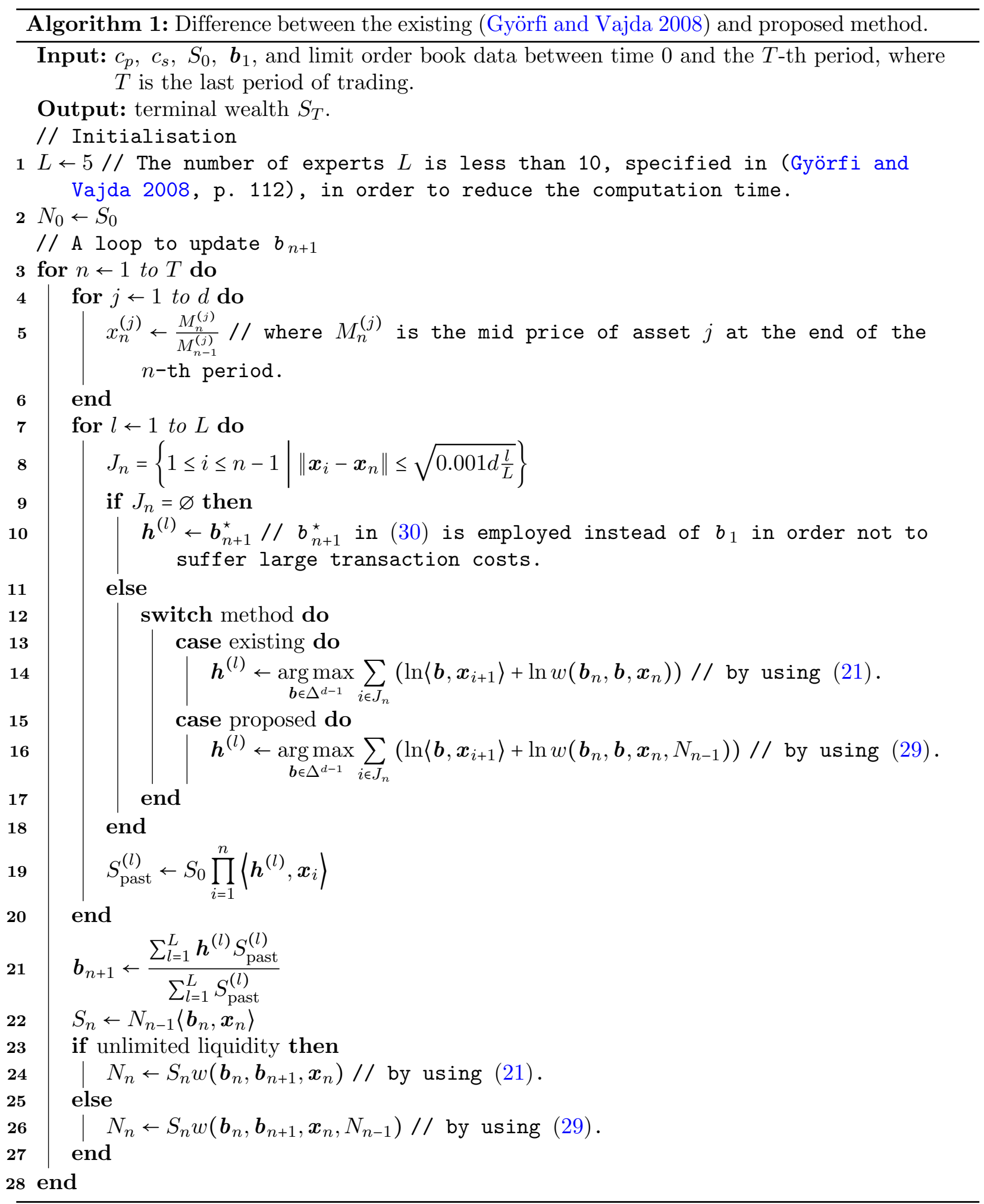

Output: terminal wealth $S_{T}$.

// Initialisation

Vajda 2008, p. 112), in order to reduce the computation time.

$N_{0} \leftarrow S_{0}$

date $b_{n+1}$

$4 \quad$ for $j \leftarrow 1$ to $d$ do

9 


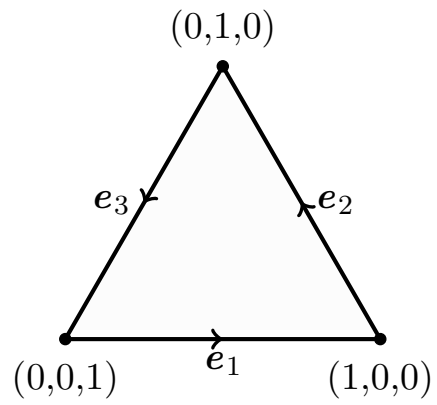

Figure 8. Ternary axes. Each vertex has the barycentric coordinate of $\boldsymbol{b} \in \Delta^{2}$, and each unit vector $\boldsymbol{e}_{j}$ corresponds to the direction of the partial derivative with respect to $b^{(j)}$.

\subsection{Local vs. global optimisation ${ }^{1}$}

Let us find which is the appropriate optimisation algorithm between local and global optimisation to maximise $\sum_{i \in J_{n}}\left(\ln \left\langle\boldsymbol{b}, \boldsymbol{x}_{i+1}\right\rangle+\ln w_{n}\right)$ in (11) with respect to $\boldsymbol{b} \in \Delta^{d-1}$. The summation part is equal to the sum of the following two equations:

$$
f(\boldsymbol{b})=\sum_{i \in J_{n}} \ln \left\langle\boldsymbol{b}, \boldsymbol{x}_{i+1}\right\rangle,
$$

and

$$
g(\boldsymbol{b})=\left|J_{n}\right| \ln w_{n}
$$

where $w_{n}$ is either $w\left(\boldsymbol{b}_{n}, \boldsymbol{b}, \boldsymbol{x}_{n}\right)$ in (21) or $w\left(\boldsymbol{b}_{n}, \boldsymbol{b}, \boldsymbol{x}_{n}, N_{n-1}\right)$ in (29), in the case of $\left|J_{n}\right| \geq 1$ (if $\left|J_{n}\right|=\varnothing$, the optimisation is not performed as the nine and tenth lines of Algorithm 1).

$f(\boldsymbol{b})$ is either (i) a constant, (ii) unimodal function of $\boldsymbol{b} \in \Delta^{d-1}$, or (iii) strictly increasing (decreasing) function of $\boldsymbol{b}$ if its first partial derivative (Figure 8 shows the direction of the partial derivative when $d=3$ )

$$
\begin{aligned}
\frac{\partial f(\boldsymbol{b})}{\partial \boldsymbol{b}} & =\left[\frac{\partial f(\boldsymbol{b})}{\partial b^{(1)}} \frac{\partial f(\boldsymbol{b})}{\partial b^{(2)}} \cdots \frac{\partial f(\boldsymbol{b})}{\partial b^{(d)}}\right]^{\mathrm{T}} \\
& =\left[\sum_{i \in J_{n}} \frac{x_{i+1}^{(1)}-x_{i+1}^{(d)}}{\left\langle\boldsymbol{b}, \boldsymbol{x}_{i+1}\right\rangle} \sum_{i \in J_{n}} \frac{x_{i+1}^{(2)}-x_{i+1}^{(1)}}{\left\langle\boldsymbol{b}, \boldsymbol{x}_{i+1}\right\rangle} \cdots \sum_{i \in J_{n}} \frac{x_{i+1}^{(d)}-x_{i+1}^{(d-1)}}{\left\langle\boldsymbol{b}, \boldsymbol{x}_{i+1}\right\rangle}\right]^{\mathrm{T}},
\end{aligned}
$$

is, respectively:

(i) $\boldsymbol{O}, \forall \boldsymbol{b} \in \Delta^{d-1}$, where $\boldsymbol{O}$ is a zero vector (a sufficient condition that makes $f(\boldsymbol{b})$ a constant is $\left.\left[\begin{array}{llll}x_{i+1}^{(1)} & x_{i+1}^{(2)} & \ldots & x_{i+1}^{(d)}\end{array}\right]^{\mathrm{T}}=\left[\begin{array}{llll}x_{i+1}^{(d)} & x_{i+1}^{(1)} & \ldots & x_{i+1}^{(d-1)}\end{array}\right]^{\mathrm{T}}, \forall i \in J_{n}\right)$

(ii) $\boldsymbol{O}$ at a single point (necessary conditions that make $f(\boldsymbol{b})$ a unimodal function are $\exists i \in J_{n}:\left[\begin{array}{llll}x_{i+1}^{(1)} & x_{i+1}^{(2)} & \ldots & x_{i+1}^{(d)}\end{array}\right]^{\mathrm{T}} \neq\left[\begin{array}{llll}x_{i+1}^{(d)} & x_{i+1}^{(1)} & \ldots & x_{i+1}^{(d-1)}\end{array}\right]^{\mathrm{T}}$, and $\left.\left|J_{n}\right| \geq 2\right)$,

(iii) $\neg \boldsymbol{O}, \forall \boldsymbol{b} \in \Delta^{d-1}$ (a necessary condition that makes $f(\boldsymbol{b})$ a strictly increasing (decreasing) function is $\left.\exists i \in J_{n}:\left[\begin{array}{lllll}x_{i+1}^{(1)} & x_{i+1}^{(2)} & \ldots & x_{i+1}^{(d)}\end{array}\right]^{\mathrm{T}} \neq\left[\begin{array}{llll}x_{i+1}^{(d)} & x_{i+1}^{(1)} & \ldots & x_{i+1}^{(d-1)}\end{array}\right]^{\mathrm{T}}\right)$.

However, $f(\boldsymbol{b})$ cannot be multimodal since it is a concave function of $\boldsymbol{b}$ (see Appendix B).

$g(\boldsymbol{b})$ is a unimodal function of $\boldsymbol{b} \in \Delta^{d-1}$ for both the cases of $w_{n}=w\left(\boldsymbol{b}_{n}, \boldsymbol{b}, \boldsymbol{x}_{n}\right)$ in (21) and $w_{n}=w\left(\boldsymbol{b}_{n}, \boldsymbol{b}, \boldsymbol{x}_{n}, N_{n-1}\right)$ in (29). This is because $w\left(\boldsymbol{b}_{n}, \boldsymbol{b}, \boldsymbol{x}_{n}\right)$ and $w\left(\boldsymbol{b}_{n}, \boldsymbol{b}, \boldsymbol{x}_{n}, N_{n-1}\right)$ are unimodal

\footnotetext{
1 Neither Györfi and Vajda (2008) nor Ormos and Urbán (2013) explained how to solve the optimisation problem of (11).
} 


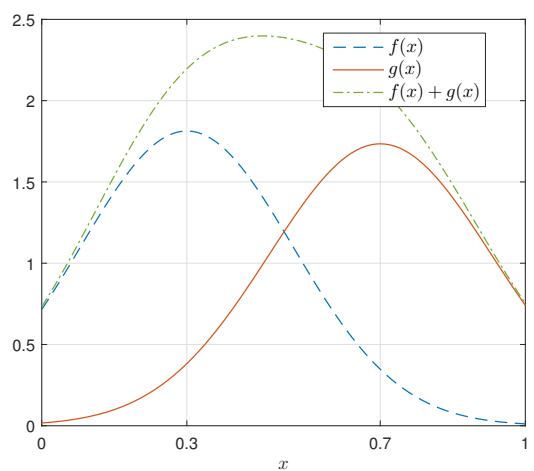

(a) $f(x)+g(x)$ is unimodal, when $f(x)$ is $\mathrm{PDF}$ of $\mathcal{N}\left(0.3,0.22^{2}\right)$, and $g(x)$ is $\mathrm{PDF}$ of $\mathcal{N}\left(0.7,0.23^{2}\right)$.

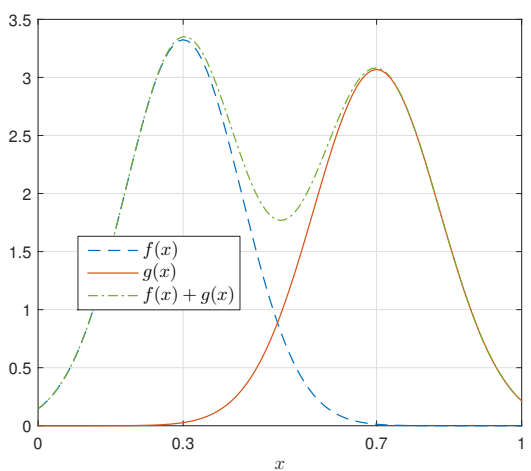

(b) $f(x)+g(x)$ is bimodal, when $f(x)$ is $\mathrm{PDF}$ of $\mathcal{N}\left(0.3,0.12^{2}\right)$, and $g(x)$ is PDF of $\mathcal{N}\left(0.7,0.13^{2}\right)$.

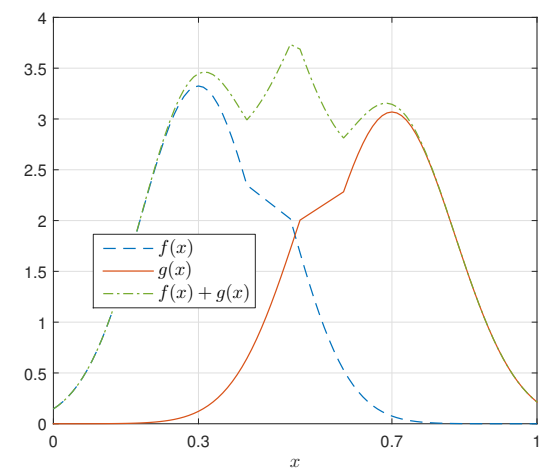

(c) $f(x)+g(x)$ is trimodal, when $f(x)$ and $g(x)$ are modified functions of Figure $9(\mathrm{~b})$.

Figure 9. The sum of two unimodal functions is not necessarily unimodal.

functions of $\boldsymbol{b}$ as explained in Section 5.3; hence, $g(\boldsymbol{b})$, the logarithmic function (which is strictly increasing) of $w_{n}$, is also unimodal.

Even though it is true that the sum of two unimodal functions is not necessarily unimodal as shown in Figure $9, f(\boldsymbol{b})+g(\boldsymbol{b})$ is either unimodal or strictly increasing (decreasing) under a certain condition:

(i) If $f(\boldsymbol{b})$ is a constant, then $f(\boldsymbol{b})+g(\boldsymbol{b})$ is unimodal without any conditions.

(ii) If $f(\boldsymbol{b})$ is unimodal, then the unimodality of the sum depends on the number of points that satisfy the equality: $\frac{\partial f(b)}{\partial b}+\frac{\partial g(b)}{\partial \boldsymbol{b}}=\boldsymbol{O}$, which denotes the local maximum of the sum. Figure $\{10(\mathrm{a}), 10(\mathrm{~b})\}$ shows an example of the sum of two unimodal functions by using $\{(21),(29)\}$, where $\arg \max _{\boldsymbol{b} \in \Delta^{1}} f(\boldsymbol{b})=\left[\begin{array}{ll}0.3 & 0.7\end{array}\right]^{\mathrm{T}}$, and $\arg \max _{b \in \Delta^{1}} g(\boldsymbol{b})=\left[\begin{array}{ll}0.7 & 0.3\end{array}\right]^{\mathrm{T}}$. Hence, there exists a possibility that the sum has multiple local maxima between 0.3 and 0.7 (e.g. Figure 9(b) and 9(c)). However, the sum has a unique local maximum at the single point: $\{0.543,0.560\}$, since the first-order partial derivative of the sum is 0 at $\{0.543,0.560\}$ as shown in Figure $\{10(\mathrm{c}), 10(\mathrm{~d})\}$. This is because both i) the opposite sign (i.e. $\frac{\partial f(\boldsymbol{b})}{\partial b^{(1)}}<0, \frac{\partial g(\boldsymbol{b})}{\partial b^{(1)}}>0, \forall b^{(1)} \in(0.3,0.7)$ ) as shown in Figure $\{10(\mathrm{c}), 10(\mathrm{~d})\}$, and ii) the crossing between $\left|\frac{\partial f(\boldsymbol{b})}{\partial b^{(1)}}\right|$ and $\left|\frac{\partial g(\boldsymbol{b})}{\partial b^{(1)}}\right|$ only once in the interval $(0.3,0.7)$ as shown in Figure $\{10(\mathrm{e}), 10(\mathrm{f})\}$. Of course, it is possible that they cross more than once in the interval since both are increasing functions in the interval (i.e. $\frac{\partial\left|\frac{\partial f(b)}{\partial b(1)}\right|}{\partial b^{(1)}}=-\frac{\partial^{2} f(\boldsymbol{b})}{\left(\partial b^{(1)}\right)^{2}}=\sum_{i \in J_{n}} \frac{\left(x_{i+1}^{(1)}-x_{i+1}^{(2)}\right)^{2}}{\left\langle\boldsymbol{b}, \boldsymbol{x}_{i+1}\right\rangle^{2}}>0, \forall b^{(1)} \in(0.3,0.7)$, and $\left.\frac{\partial\left|\frac{\partial g(b)}{\partial b^{(1)}}\right|}{\partial b^{(1)}}=\frac{\partial^{2} g(\boldsymbol{b})}{\left(\partial b^{(1)}\right)^{2}}>0, \forall b^{(1)} \in(0.3,0.7)^{1}\right)$. However, if the proportional TC rate is low (technically, low $\frac{c_{p}+c_{s}}{1+c_{p}}$ from $\left.(\mathrm{C} 8)\right)$, the second partial derivative of $g(\boldsymbol{b})$ is approximated as 0 (i.e. $\frac{\partial^{2} g(\boldsymbol{b})}{\left(\partial b^{(1)}\right)^{2}} \approx 0$ ), which in turn makes $\left|\frac{\partial g(\boldsymbol{b})}{\partial b^{(1)}}\right|\{$ a constant, piecewise constant function $\}$ in the case of $\{(21),(29)\}$ as shown in Figure $\{10(\mathrm{e}), 10(\mathrm{f})\}$. As a result, $\left|\frac{\partial f(\boldsymbol{b})}{\partial b^{(1)}}\right|$ and $\left|\frac{\partial g(\boldsymbol{b})}{\partial b^{(1)}}\right|$ cross only once in the interval $(0.3,0.7)$ when $\frac{c_{p}+c_{s}}{1+c_{p}}$ is low, which in turn makes $f(\boldsymbol{b})+g(\boldsymbol{b})$ unimodal.

1 The proof of $\frac{\partial^{2} g(b)}{\left(\partial b^{(1)}\right)^{2}}>0$ is in Appendix C in the case of (21), but no proof of $\frac{\partial^{2} g(b)}{\left(\partial b^{(1)}\right)^{2}}>0$ is provided in this paper in the case of (29). Meanwhile, $\frac{\partial^{2} g(b)}{\left(\partial b^{(1)}\right)^{2}}>0$ is observed at differentiable points in Figure 10(b) by using the numerical differentiation: $\frac{\partial^{2} g(b)}{\left(\partial b^{(1)}\right)^{2}} \approx \frac{g\left(b+[h-h]^{\mathrm{T}}\right)-2 g(b)+g\left(b-[h-h]^{\mathrm{T}}\right)}{h^{2}}$, where $h=10^{-3}$. 


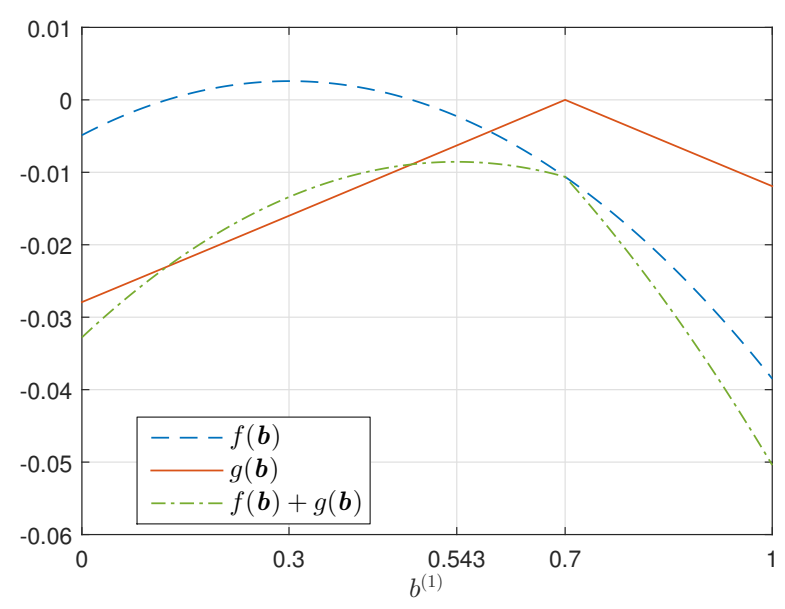

(a) $g(\boldsymbol{b})=2 \ln w_{n}$, where $w_{n}=\left(\boldsymbol{b}_{n}, \boldsymbol{b}, \boldsymbol{x}_{n}\right)$ in $(21)$.

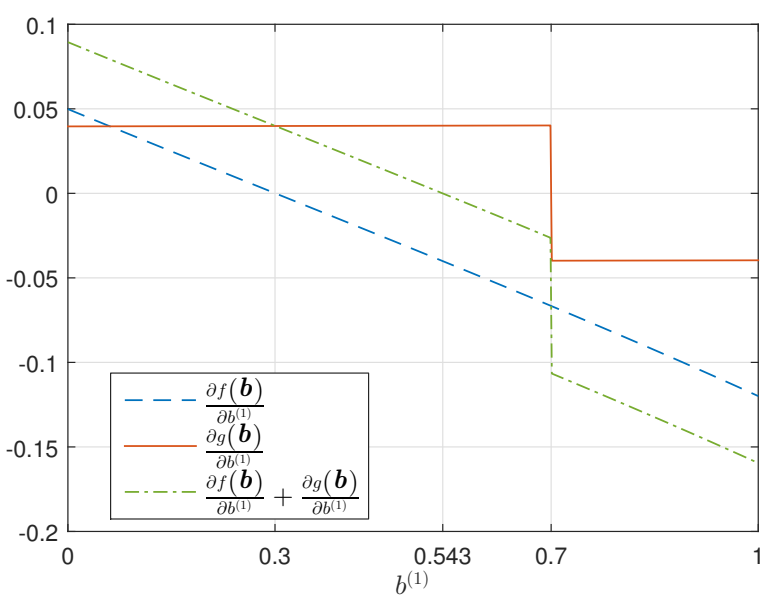

(c) First-order numerical differentiation of the upper figure.

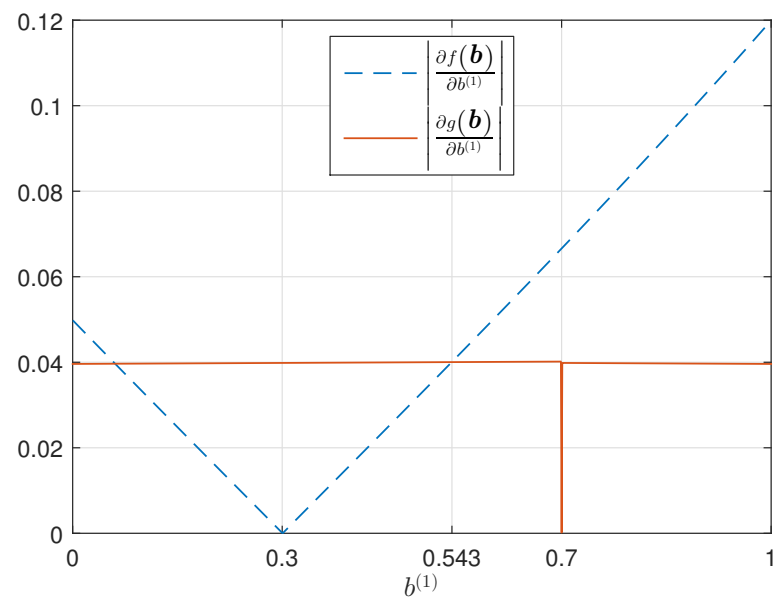

(e) Absolute value of the upper figure.

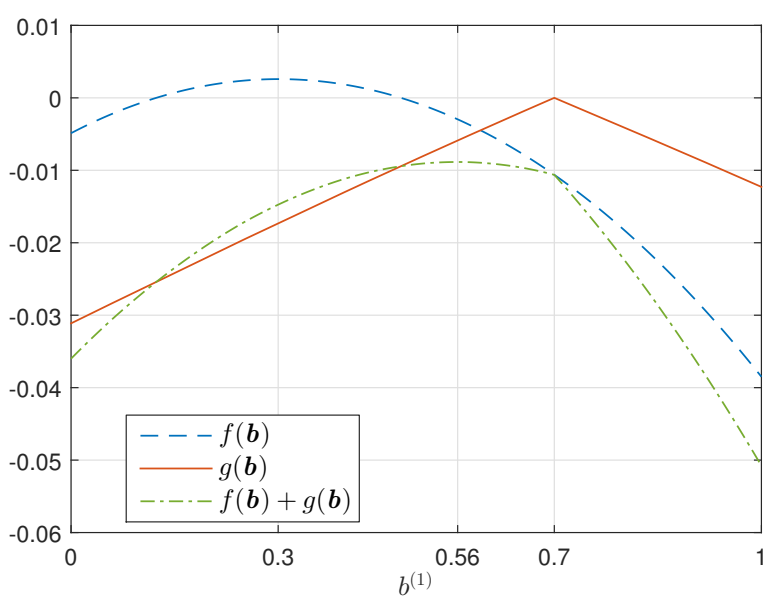

(b) $g(\boldsymbol{b})=2 \ln w_{n}$, where $w_{n}=\left(\boldsymbol{b}_{n}, \boldsymbol{b}, \boldsymbol{x}_{n}, N_{n-1}=10^{7}\right)$ in (29). Limit order book data of $\operatorname{INTC}\left(b^{(1)}\right)$ and $\operatorname{MSFT}\left(b^{(2)}\right)$ on 21 Jun 2012 at 16:00:00 was used.

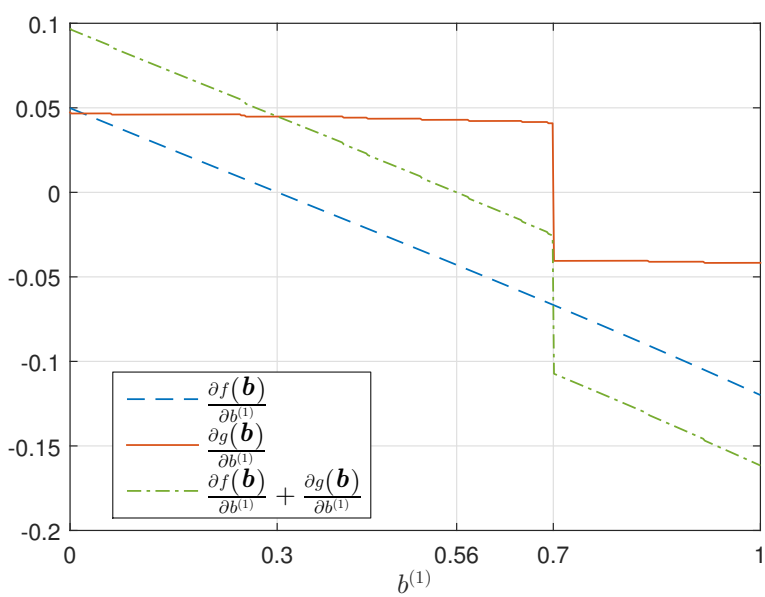

(d) First-order numerical differentiation of the upper figure.

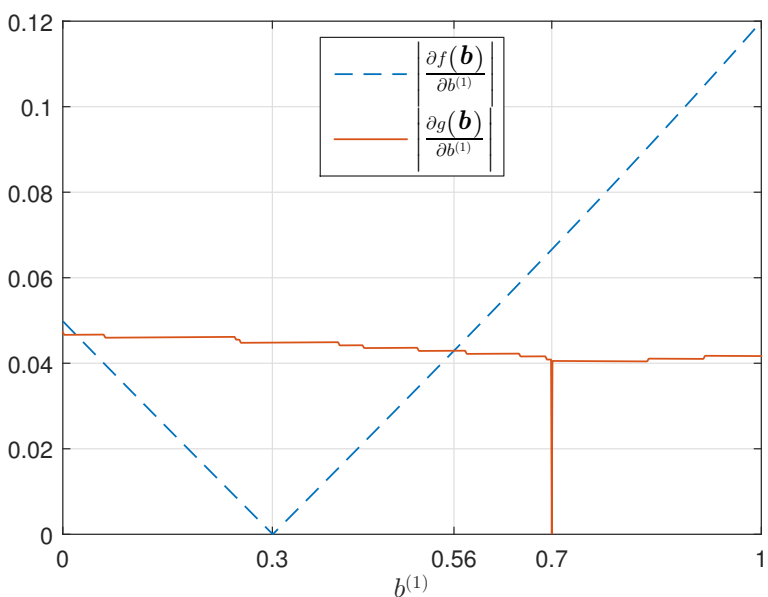

(f) Absolute value of the upper figure.

Figure 10. If $f(\boldsymbol{b})$ is unimodal, then $f(\boldsymbol{b})+g(\boldsymbol{b})$ is also unimodal under low proportional transaction cost rates $\left(f(b)=\ln \left\langle\boldsymbol{b},\left[\begin{array}{ll}1.151 & 0.876\end{array}\right]^{\mathrm{T}}\right\rangle+\ln \left\langle\boldsymbol{b},\left[\begin{array}{ll}0.836 & 1.136\end{array}\right]^{\mathrm{T}}\right\rangle, c_{p}=c_{s}=1 \%, \boldsymbol{b}_{n}=\left[\begin{array}{ll}0.7 & 0.3\end{array}\right]^{\mathrm{T}}\right.$, and $\left.\boldsymbol{x}_{n}=\left[\begin{array}{ll}1.1 & 1.1\end{array}\right]^{\mathrm{T}}\right)$. More nondifferentiable points are observed in Figure $\{10(\mathrm{~d}), 10(\mathrm{f})\}$ than Figure $\{10(\mathrm{c}), 10(\mathrm{e})\}$ because the average market impact cost function is piecewise as shown in Figure 4 . 
(iii) If $f(\boldsymbol{b})$ is strictly increasing (decreasing), then $f(\boldsymbol{b})+g(\boldsymbol{b})$ is either unimodal or strictly increasing (decreasing) under the same condition of low proportional TC rates as (ii). Even if there exists an interval of the opposite sign between $\frac{\partial f(\boldsymbol{b})}{\partial b^{(1)}}$ and $\frac{\partial g(\boldsymbol{b})}{\partial b^{(1)}}$, their absolute values cross at most once if the latter is almost constant.

Consequently, $f(\boldsymbol{b})+g(\boldsymbol{b})$ is either unimodal or strictly increasing (decreasing) under the sufficient condition of low proportional TC rates (i.e. low $\frac{c_{p}+c_{s}}{1+c_{p}}$ ), which implies that a local maximum is unique. Thus, the local optimum is guaranteed to be the global optimum when $\frac{c_{p}+c_{s}}{1+c_{p}}$ is low.

\subsection{Initial value of optimisation}

$\boldsymbol{b}_{n+1}^{\star}$ in (30) is recommended as the initial value of local optimisation, which makes $w_{n}=1$ (i.e. $\boldsymbol{b}_{n+1}^{\star}$ is the portfolio vector of zero TCs by not rebalancing a portfolio), at the end of the $n$-th period. This is in order to reduce the computation time of the optimisation: calculating $g(\boldsymbol{b})=\left|J_{n}\right| \ln w_{n}$ in (40) with TCF $w_{n}=w\left(\boldsymbol{b}_{n}, \boldsymbol{b}, \boldsymbol{x}_{n}, N_{n-1}\right)$ in (29) requires using the price and volume data of LOBs between the first absolute level of LOB and a higher absolute level of LOB (a positive level means buying stocks, whereas a negative level means selling stocks) as $\boldsymbol{b}$ is farther away from $\boldsymbol{b}_{n+1}^{\star}$, which in turn causes a heavier burden to calculate (14). If the initial value is $\boldsymbol{b}_{n+1}^{\star}$, the heavy computation can be avoided at least at the early stage of the optimisation.

\section{Simulations (backtesting)}

Monte Carlo (MC) simulations consisting of independent trials of random stock selection - each stock has an equal chance of being selected-were conducted to compare the performance among the existing methods and the proposed method. This is similar to (Kozat and Singer 2011, Section 4), while the differences are i) the number of trials, increased from 50 to 100 in order to generate more reliable results, and ii) the number of selected stocks, increased from 3 to 30 in order to generate more practical results.

10-level (i.e. 10 levels of the ask side and 10 levels of the bid side) historical LOB data ${ }^{1}$ of NASDAQ 100 Index Components ${ }^{2}$ (30 components are randomly selected among the 100 components at each trial) between 1 Jan 2008 and 31 Dec 2015 (total 2015 trading days) was downloaded from Limit Order Book System: The Efficient Reconstructor (LOBSTER), ${ }^{3}$ and the LOB data was sampled with the period of one day at the end of regular NASDAQ stock market trading (i.e. 4:00:00 p.m. Eastern Time). ${ }^{4}$ Also, all OPS methods in this section rebalanced a portfolio at the closing time on every U.S. trading day (i.e. rebalancing once a trading day).

The 8-year period of the NASDAQ data set is much shorter than the 44-year period of an NYSE data set between 1962 and 2006, ${ }^{5}$ used in (Györfi and Vajda 2008, Györfi and Walk 2012, Horváth and Urbán 2012) for their experiments. Hence, the pattern-matching-based OPS methods (e.g. Györfi and Vajda (2008)'s and the proposed method) with the 8-year data might suffer from low performance due to insufficient data to discover the tendencies of the market vectors, as similarly

1 If accessing LOB data at greater than level 10 is required, ask price and volume at level $i \in \mathbb{Z}_{>10}$ are estimated as $P_{i}=P_{10}+\frac{P_{10}-P_{-1}}{10}(i-10), V_{i}=\frac{\sum_{k=1}^{10} V_{k}}{10}$, respectively. Similarly, if accessing LOB data at less than level -10 is required, bid price and volume at level $i \in \mathbb{Z}_{<-10}$ are estimated as $P_{i}=P_{-10}+\frac{P_{-10}-P_{1}}{10}(-i-10), V_{i}=\frac{\sum_{k=1}^{10} V_{-k}}{10}$, respectively.

2 Historical, not current, NASDAQ 100 Index Components on 1 Jan 2008 was downloaded from http:// marketcapitalizations.com/historical-data/historical-components-nasdaq/.

3 LOBSTER (https://lobsterdata.com/) has LOB data from 27 Jun 2007 to the present, and the LOB data of LOBSTER does not include hidden LOBs (Huang and Polak 2011, Table 1).

4 1:00:00 p.m. data was used for the following NASDAQ early closing dates: 3 Jul 2008, 28 Nov 2008, 24 Dec 2008,27 Nov 2009, 24 Dec 2009, 26 Nov 2010, 25 Nov 2011, 3 Jul 2012, 23 Nov 2012, 24 Dec 2012, 3 Jul 2013, 29 Nov 2013, 24 Dec 2013, 3 Jul 2014, 28 Nov 2014, 24 Dec 2014, 27 Nov 2015, and 24 Dec 2015.

5 The NYSE data is downloadable at http://www.cs.bme.hu/ oti/portfolio/data.html. 
observed in (Györfi et al. 2006, Table 4.1). Consequently, the two-stage splitting scheme (Györfi et al. 2012, p. 102): ${ }^{1}$

(i) Learning phase (that ranges between 1 Jan 1998 and 31 Dec 2007): only data collection is conducted without portfolio rebalancing;

(ii) Concurrent phase (that ranges between 1 Jan 2008 and 31 Dec 2015): both data collection and portfolio rebalancing using the learning and concurrent phases are conducted concurrently;

was employed in the following experiments for Györfi and Vajda (2008)'s and the proposed method.

The actual number of stock candidates is $100-34-5=61$ by the splitting: 34 companies listed on NASDAQ after 1 Jan 1998 (their historical data is not enough for the learning phase), and 5 companies delisted (ALTR, AMLN, DELL, and TLAB were acquired by other companies, and FWLT was voluntarily delisted from NASDAQ) before 31 Dec 2015 (their historical data is not enough for the concurrent phase). Therefore, the number of possible portfolio combinations is $\left(\begin{array}{l}61 \\ 30\end{array}\right)=2.3 \times 10^{17}$, and the portion of $\frac{100}{2.3 \times 10^{17}}=4.3 \times 10^{-16}$ is covered by the MC simulations.

The range of proportional TC rate was set as $c_{p}=0$ and $0.00184 \% \leq c_{s} \leq 0.5 \%$ (i.e. $0.00184 \% \leq \frac{c_{p}+c_{s}}{1+c_{p}} \leq 0.5 \%$ ). This is because the securities transaction tax rates in most of the G20 countries vary between $0.1 \%$ and $0.5 \%$ (Matheson 2011), and those in the United States in 2015 are $c_{p}=0$ and $c_{s}=0.184 \mathrm{bps}^{2}$ However, stock brokerage commissions were ignored by an assumption that institutional investors, who pay tiny commissions, rather than individual investors, are the main users of OPS.

When calculating the price relative of asset $j$ of the $n$-th day, cash dividends, stock dividends, and stock splits should be considered as

$$
x_{n}^{(j)}=\frac{M_{n}^{(j)} \frac{A_{n}^{(j)}}{C_{n}^{(j)}}}{M_{n-1}^{(j)} \frac{A_{n-1}^{(j)}}{C_{n-1}^{(j)}}},
$$

where $\left\{M_{n}^{(j)}, C_{n}^{(j)}, A_{n}^{(j)}\right\}$ is the $\{$ closing mid, closing, adjusted closing $\}$ price from $\{$ LOBSTER, Yahoo Finance, Yahoo Finance $\}$ of asset $j$ of the $n$-th day. However, the proportion of the data mismatching between LOBSTER and Yahoo Finance (i.e. $\left(P_{1} \neq C\right) \vee\left(P_{-1} \neq C\right)$, where $P_{1}\left(P_{-1}\right)$ is the best ask (bid) price at the closing time from LOBSTER, and $C$ is the closing price from Yahoo Finance) for the 55 stocks and 2015 trading days was as high as $69.6 \%$. Therefore, the price relative of asset $j$ of the $n$-th day was calculated as

$$
x_{n}^{(j)}=\frac{A_{n}^{(j)}}{A_{n-1}^{(j)}} .
$$

The following MC simulations are categorised into two parts according to whether the liquidity of assets is unlimited or limited. The first is to provide a benchmark for the comparison between the ideal assumption (i.e. unlimited liquidity) and the real stock market (i.e. limited liquidity), and the second is to demonstrate both i) the performance deterioration of OPS by MICs and ii) the superiority of the proposed method in the environment of the limited liquidity. Additionally, graphical comparisons and computation time analysis are provided.

The MATLAB codes of the following experiments have been uploaded on http://www . mathworks . com/matlabcentral/fileexchange/56496 to avoid any potential ambiguity of the MC simula-

\footnotetext{
1 The original intention to introduce the two-stage splitting scheme by Györfi et al. (2012) is to prove its uselessness by the assumption of the stationarity and long-term investment.

2 Order making fiscal year 2015 annual adjustments to transaction fee rates, U.S. Securities and Exchange Commission [Release No. 34-74057/15 Jan 2015].
} 


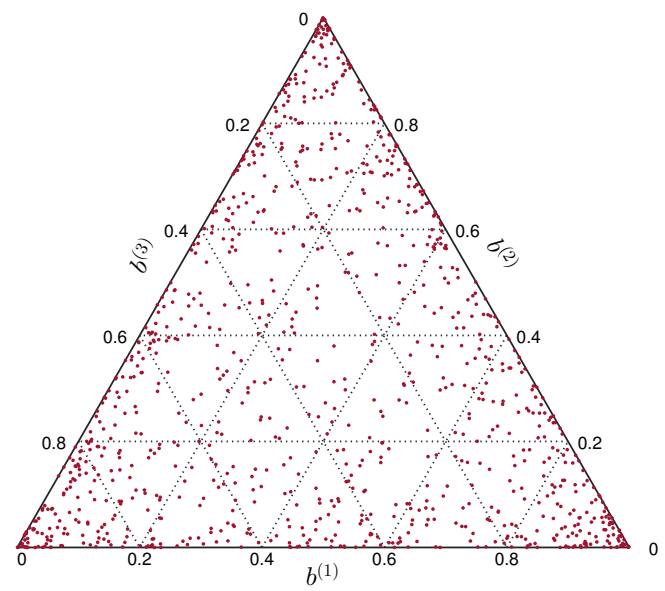

Figure 11. An example scatter plot of the Dirichlet distribution with $\boldsymbol{\alpha}=\left[\begin{array}{lll}1 / 2 & 1 / 2 & 1 / 2\end{array}\right]^{\mathrm{T}}$ and $K=10^{3}$.

tions. ${ }^{1}$ You may leave comments on the web page; any feedback or bug report is welcome.

\subsection{In the case that the liquidity of assets is unlimited}

In the case that the liquidity of assets is unlimited, TCF $w_{n}$ in (21) is employed to calculate both the portfolio vector of next day $\boldsymbol{b}_{n+1}$ and net wealth $N_{n}$ in (18) at the end of every day, but the LOB data is disregarded.

8.1.1. Comparison between (Cover 1991) and (Blum and Kalai 1999). Since performance comparison between (Cover 1991) and (Blum and Kalai 1999) had not been conducted by Blum and Kalai (1999), it was carried out in this paper. Their difference (i.e. Blum and Kalai (1999) takes into account $c_{p}$ and $c_{s}$ when calculating $\boldsymbol{b}_{n+1}$, whereas Cover (1991) does not) is described in Algorithm 2 in Appendix A.

The numerical integral in both (2) and (9) is performed by using MC methods. Let $\boldsymbol{b}(k)$ be the $k$-th $(k \in\{1,2, \ldots, K\})$ random numbers of the Dirichlet distribution with the $d$-dimensional concentration parameter vector $\boldsymbol{\alpha}=\left[\begin{array}{llll}1 / 2 & 1 / 2 \ldots 1 / 2\end{array}\right]^{\mathrm{T}}$, where $K=10^{3}$ is the number of samplings (Figure 11 shows an example). The portfolio vector in (2) is then approximated as (Ishijima 2001)

$$
\boldsymbol{b}_{n+1}=\frac{\sum_{k=1}^{K} \boldsymbol{b}(k) S_{n}\left(\boldsymbol{b}(k), \boldsymbol{x}_{1: n}\right)}{\sum_{k=1}^{K} S_{n}\left(\boldsymbol{b}(k), \boldsymbol{x}_{1: n}\right)} .
$$

Table 2 shows the performance comparison between the two methods by using the annualised return:

$$
\left(\frac{S_{T}}{S_{0}}\right)^{\frac{252}{T}}-1
$$

where $T$ is the number of total trading days, and 252 is the number of trading days in a year. Even though the negative differences between the two means for all $c_{s}$ imply that the Cover's method is better than the Blum's method, the statistical significance of the difference in means by unpaired

\footnotetext{
1 MATLAB fmincon (a local, not global, optimisation solver), which finds minimum of constrained nonlinear multivariable function, was utilised to solve (11) for both the existing method with (21) and the proposed method with (29). MATLAB $\mathrm{R} 2011 \mathrm{~b}$ and R2014b resulted in slightly different solutions of $\boldsymbol{h}^{(l)}\left(\boldsymbol{x}_{1: n}\right)$ in $(11)$, and the older version was used to perform the simulations of this paper.
} 
Table 2. Statistics of annualised returns for comparison between Cover and Blum's method when market liquidity is unlimited $\left(c_{p}=0\right)$.

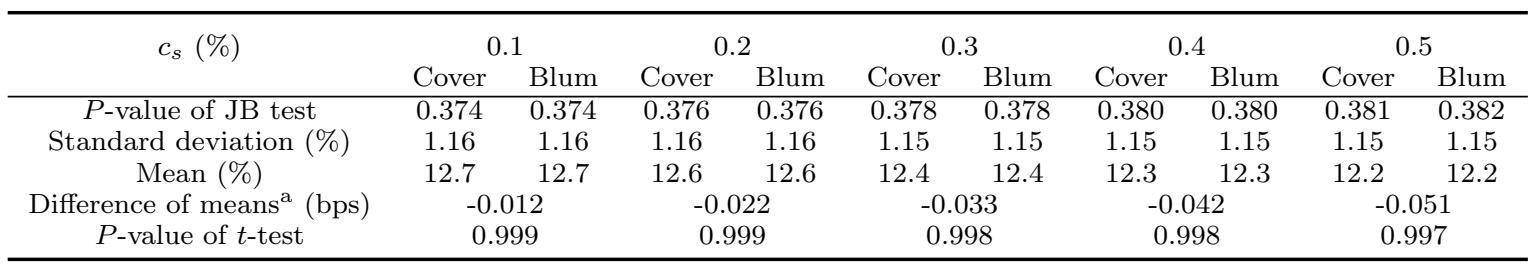

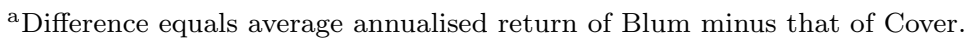

two-sample $t$-tests with unequal variances, whose null hypothesis is that the data in two groups comes from independent random samples from normal distributions with equal means but different variances, is negligible for all $c_{s},{ }^{1}$ where the assumption of the normal distribution was tested by the Jarque-Bera (JB) tests.

The $t$-tests (the $t$-test denotes the unpaired two-sample $t$-test with unequal variances here and in the remainder of this paper) answer whether the performance difference is fundamental or whether it is due to random fluctuations (Simon 2013, p. 631). In other words, if a $p$-value of the $t$-test is less than a significance level (e.g. 0.05), the performance difference is fundamental. ${ }^{2}$ However, the $p$-values of the $t$-tests in Table 2 are greater than or equal to 0.05 for all $c_{s}$, which means that the performance difference between the Cover's method and the Blum's method is due to random fluctuations. As a result, the Blum's method is not compared to any other methods in the subsequent experiments.

8.1.2. Comparison among (Kozat and Singer 2011), (Cover 1991), and (Györfi and Vajda 2008). The $t$-tests were performed to compare the performance between a benchmark and OPS methods (Table 3 shows the results), where a strategy of buy-and-hold (B\&H) with the initial portfolio $\boldsymbol{b}_{1}$ is the benchmark against all OPS methods, and its performance is independent of $c_{p}$ or $c_{s}$ as it does not incur any TCs. The Kozat, Cover, and Györfi's method show the positive differences with $p$-values less than 0.01 when $c_{s} \leq 0.5 \%$, which means that these OPS methods are highly fundamentally superior to $\mathrm{B} \& \mathrm{H}$ when $c_{s} \leq 0.5 \%$.

However, Wilcoxon rank sum tests (also called Wilcoxon-Mann-Whitney tests), whose null hypothesis is that data in two groups are samples from continuous distributions (even non-normal distributions) with equal medians, were not performed even though the violation of the normality assumption of the $t$-test was confirmed by the JB test with the significance level of 0.05 as shown in Table 3 ( $p$-values of the JB test less than 0.05 are marked in bold, which implies non-normality). This is because the $t$-test is superior to the Wilcoxon rank sum test when variances (i.e. the variance of annualised returns of B\&H and that of each OPS method) differ (Skovlund and Fenstad 2001).

Standard deviation in Table 3 can be interpreted as the sensitivity of the annualised return to the random stock selection: the Kozat's method and the Györfi's method are the least and most sensitive, respectively, for all $c_{s}$. In addition, Figure 13 (Figure 12) shows the decreasing trend of the performance as $c_{s}$ increases by using the mean (box plots) of annualised returns of each method: the Kozat's method and the Györfi's method show the least and most decreasing trend, respectively.

1 Although the sample standard deviations at each $c_{s}$ in Table 2 are similar, unpaired two-sample $t$-tests with equal variance, whose null hypothesis is that the data in two groups comes from independent random samples from normal distributions with equal means and equal but unknown variances, were not performed. This is because even when the variances are identical, the unequal variance $t$-test performs just as effectively as the equal variance $t$-test in terms of Type I error (Ruxton 2006 ).

2 The $p$-value of the $t$-test is interpreted as the probability that a difference in the mean values would be obtained, given that the population means of two methods are equivalent. I.e. the $p$-value is not equal to the probability that the population means are equivalent (Simon 2013, p. 635). 
Table 3. Statistics of annualised returns for comparison among Kozat, Cover, and Györfi's method when market liquidity is unlimited $\left(c_{p}=0\right)$.

\begin{tabular}{ccccccccccc}
\hline$c_{s}(\%)$ & & 0.00184 & 0.00184 & 0.00184 & 0.1 & 0.1 & 0.1 & 0.2 & 0.2 & 0.2 \\
& B\&H & Kozat & Cover & Györfi & Kozat & Cover & Györfi & Kozat & Cover & Györfi \\
\hline$P$-value of JB test & 0.419 & 0.603 & 0.768 & $\mathbf{0 . 0 3 8}$ & 0.602 & $0.76^{* * *}$ & $\mathbf{0 . 0 1 1}$ & 0.603 & 0.758 & $\mathbf{0 . 0 0 9}$ \\
Standard deviation (\%) $^{1.15}$ & 1.19 & 1.34 & 7.39 & 1.19 & 1.34 & 6.98 & 1.19 & 1.33 & 5.74 \\
Mean (\%) & 11.3 & 12.1 & 12.8 & 21.4 & 12.1 & 12.7 & 17.6 & 12.1 & 12.6 & 16.1 \\
Difference of means $^{\mathrm{a}}(\%)$ & - & $0.80^{* * *}$ & $1.54^{* * *}$ & $10.10^{* * *}$ & $0.79^{* * *}$ & $1.40^{* * *}$ & $6.28^{* * *}$ & $0.79^{* * *}$ & $1.25^{* * *}$ & $4.82^{* * *}$ \\
$P$-value of $t$-test & - & $2.7 \times 10^{-6}$ & $9.7 \times 10^{-16}$ & $1.4 \times 10^{-24}$ & $3.3 \times 10^{-6}$ & $1.6 \times 10^{-13}$ & $2.1 \times 10^{-14}$ & $4.0 \times 10^{-6}$ & $2.3 \times 10^{-11}$ & $4.7 \times 10^{-13}$ \\
\hline
\end{tabular}

\begin{tabular}{|c|c|c|c|c|c|c|c|c|c|}
\hline$c_{s}(\%)$ & $\begin{array}{c}0.3 \\
\text { Kozat }\end{array}$ & $\begin{array}{c}0.3 \\
\text { Cover } \\
\end{array}$ & $\begin{array}{c}0.3 \\
\text { Györfi } \\
\end{array}$ & $\begin{array}{c}0.4 \\
\text { Kozat }\end{array}$ & $\begin{array}{c}0.4 \\
\text { Cover } \\
\end{array}$ & $\begin{array}{c}0.4 \\
\text { Györfi }\end{array}$ & $\begin{array}{c}0.5 \\
\text { Kozat }\end{array}$ & $\begin{array}{c}0.5 \\
\text { Cover } \\
\end{array}$ & $\begin{array}{c}0.5 \\
\text { Györfi }\end{array}$ \\
\hline$P$-value of JB test & 0.603 & 0.753 & 0.081 & 0.604 & 0.750 & 0.063 & 0.602 & 0.747 & 0.066 \\
\hline Standard & 1.1 & 1.33 & 5.62 & 1.19 & 1.33 & 5.81 & 1.19 & 1.33 & 5.89 \\
\hline Mea & 12.1 & 12.4 & 15.0 & 12.1 & 12.3 & 14.5 & 12.1 & 12.1 & 14.0 \\
\hline Difference of means ${ }^{\mathrm{a}}(\%)$ & $0.78^{* * *}$ & $1.10^{* * *}$ & $3.69^{* * *}$ & $0.77^{* * *}$ & $0.95^{* * *}$ & $3.20^{* * *}$ & $0.76^{* * *}$ & $0.80^{* * *}$ & $2.69^{* * *}$ \\
\hline$P$-value of $t$-test & $4.8 \times 10^{-6}$ & $2.5 \times 10^{-9}$ & $3.5 \times 10^{-9}$ & $5.9 \times 10^{-6}$ & $1.8 \times 10^{-7}$ & $4.1 \times 10^{-7}$ & $7.1 \times 10^{-6}$ & $8.7 \times 10^{-6}$ & $1.8 \times 10^{-5}$ \\
\hline
\end{tabular}

${ }^{a}$ Difference equals average annualised return of the corresponding method at each $c_{s}$ minus that of buy-and-hold $(\mathrm{B} \& \mathrm{H}) .{ }^{*} p<0.1 ;{ }^{* *} p<0.05 ;{ }^{* * *} p<0.01$.
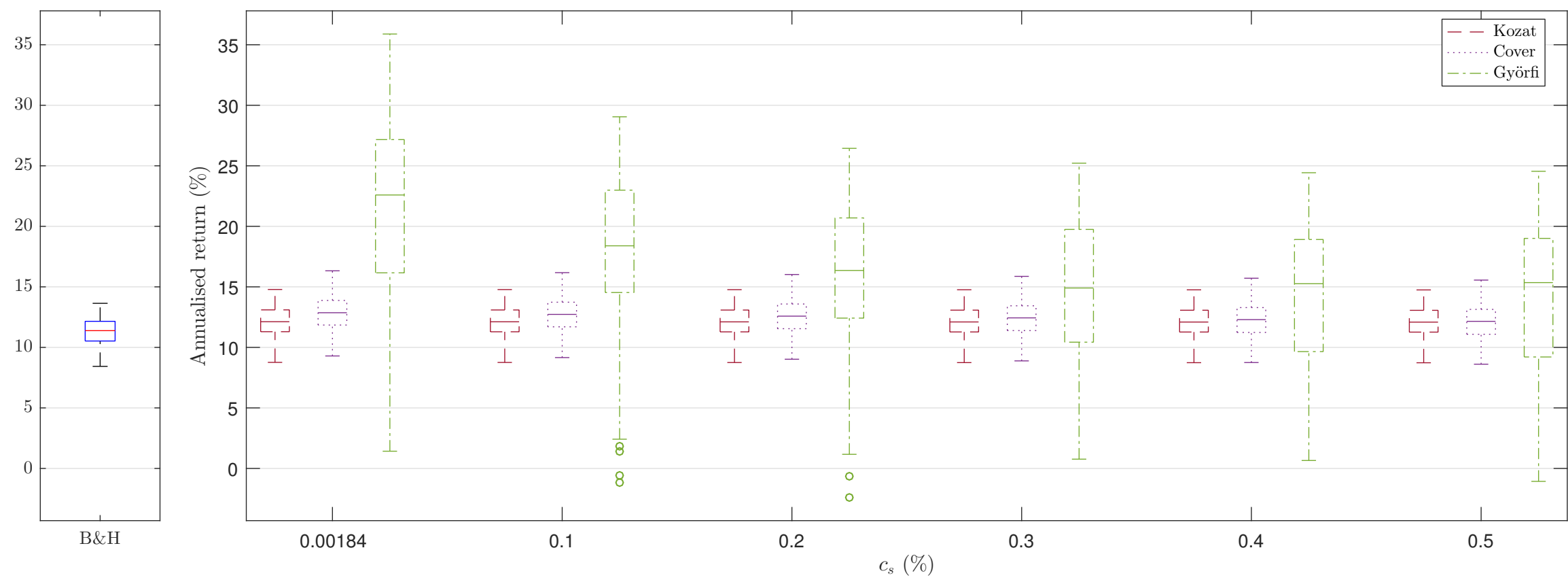

Figure 12. Box plots of annualised returns for comparison among Kozat, Cover, and Györfi's method when market liquidity is unlimited $\left(c_{p}=0\right)$. 


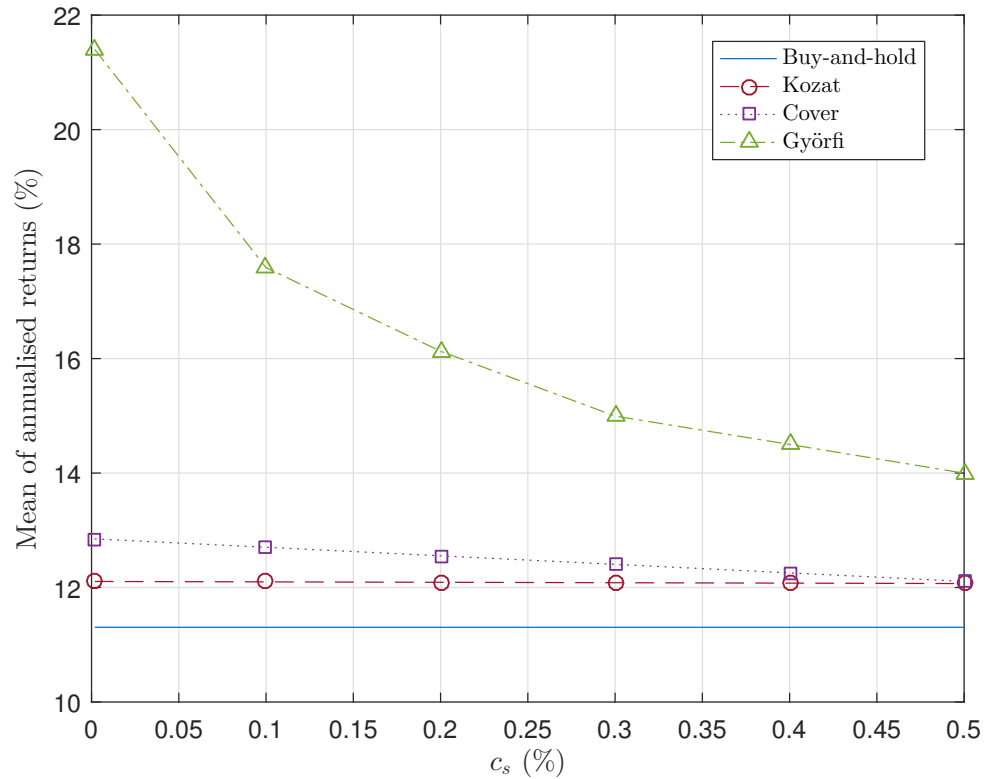

Figure 13. Mean of annualised returns for comparison among Kozat, Cover, and Györfi's method when market liquidity is unlimited $\left(c_{p}=0\right)$.

Figure 12 shows that the higher expected return does not always guarantee the higher profits. In other words, there is no best OPS method, but investors may choose a preferable OPS method by considering both the expected return and the risk (i.e. standard deviation of annualised returns). Meanwhile, negative returns are observed in the low outliers of the Györfi's method when $c_{s} \geq 0.1 \%$, resulting in the loss of money (i.e. $S_{T}<S_{0}$ ).

\subsection{In the case that the liquidity of assets is limited}

In the case that the liquidity of assets is limited, TCF $w_{n}$ in (29) is employed to calculate the net wealth $N_{n}$ in (18) at the end of every day. Therefore, the performance of all OPS methods depends

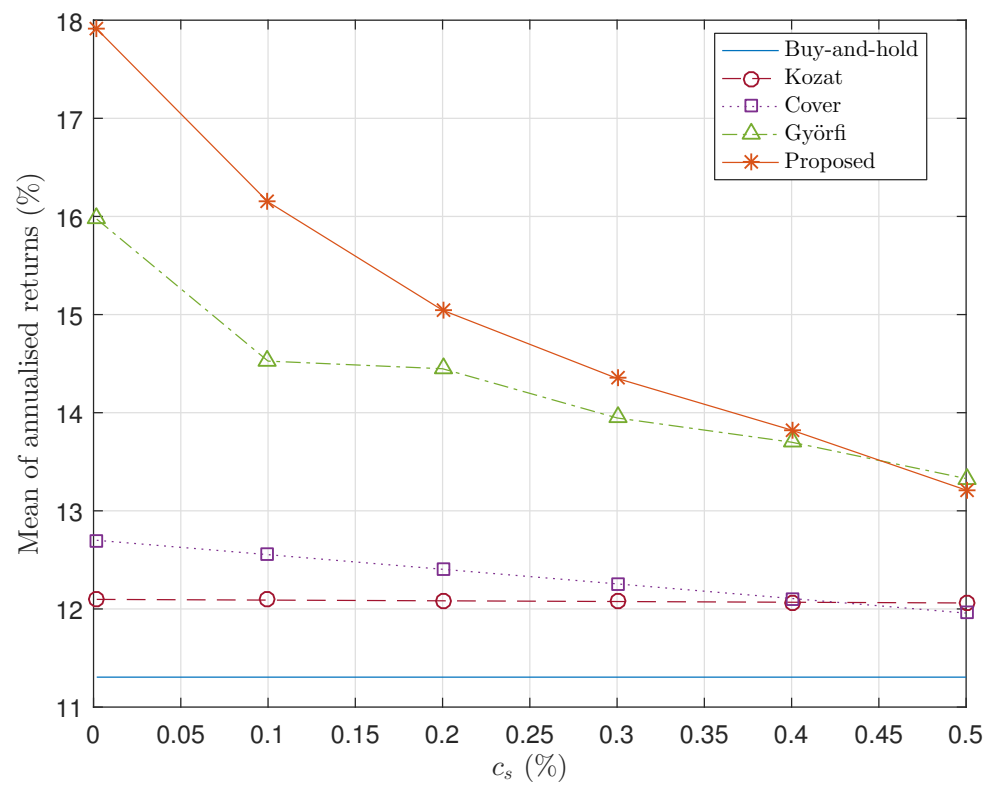

Figure 14. Mean of annualised returns for comparison among Kozat, Cover, Györfi's, and proposed method when market liquidity is limited and $S_{0}=10^{4}\left(c_{p}=0\right)$. 
Table 4. Statistics of annualised returns for comparison among Kozat, Cover, Györfi's, and proposed method when market liquidity is limited and $S_{0}=10^{4}\left(c_{p}=0\right)$.

\begin{tabular}{|c|c|c|c|c|c|c|c|c|c|c|c|c|c|}
\hline$c_{s}(\%)$ & $\mathrm{B} \& \mathrm{H}$ & $\begin{array}{c}0.00184 \\
\text { Kozat }\end{array}$ & $\begin{array}{c}0.00184 \\
\text { Cover }\end{array}$ & $\begin{array}{c}0.00184 \\
\text { Györfi }\end{array}$ & $\begin{array}{c}0.00184 \\
\text { Proposed }\end{array}$ & $\begin{array}{c}0.1 \\
\text { Kozat }\end{array}$ & $\begin{array}{c}0.1 \\
\text { Cover }\end{array}$ & $\begin{array}{c}0.1 \\
\text { Györfi }\end{array}$ & $\begin{array}{c}0.1 \\
\text { Proposec }\end{array}$ & $\begin{array}{c}0.2 \\
\text { Kozat }\end{array}$ & $\begin{array}{c}0.2 \\
\text { Cover }\end{array}$ & $\begin{array}{c}0.2 \\
\text { Györfi }\end{array}$ & $\begin{array}{c}0.2 \\
\text { Proposed }\end{array}$ \\
\hline$P$-value of JB test & 0.419 & 0.601 & 0.735 & 0.009 & 0.027 & 0.602 & 0.728 & 0.009 & 0.016 & 0.604 & 0.723 & 0.020 & 0.149 \\
\hline Standard deviation (\%) & 1.15 & 1.19 & 1.33 & 8.50 & 7.00 & 1.19 & 1.33 & 7.81 & 5.89 & 1.19 & 1.32 & 5.92 & 5.38 \\
\hline Mean $(\%)$ & 11.3 & 12.1 & 12.7 & 16.0 & 17.9 & 12.1 & 12.6 & 14.5 & 16.1 & 12.1 & 12.4 & 14.4 & 15.0 \\
\hline Difference of means ${ }^{\mathrm{a}}(\%)$ & - & $0.79^{* * *}$ & $1.39^{* * *}$ & $4.67^{* * *}$ & $6.61^{* * *}$ & $0.79^{* * *}$ & $1.25^{* * *}$ & $3.22^{* * *}$ & $4.84^{* * *}$ & $0.78^{* * *}$ & $1.10^{* * *}$ & $3.14^{* * *}$ & $3.74^{* * *}$ \\
\hline$P$-value of $t$-test & - & $3.4 \times 10^{-6}$ & $1.6 \times 10^{-13}$ & $3.6 \times 10^{-7}$ & $2.3 \times 10^{-15}$ & $4.1 \times 10^{-6}$ & $2.2 \times 10^{-11}$ & $8.8 \times 10^{-5}$ & $1.1 \times 10^{-12}$ & $5.0 \times 10^{-}$ & $2.3 \times 10^{-\mathrm{S}}$ & $9.4 \times 10^{-7}$ & $6.0 \times 10^{-10}$ \\
\hline \multicolumn{2}{|l|}{$c_{s}(\%)$} & $\begin{array}{c}0.3 \\
\text { Kozat }\end{array}$ & $\begin{array}{c}0.3 \\
\text { Cover }\end{array}$ & $\begin{array}{c}0.3 \\
\text { Györfi }\end{array}$ & $\begin{array}{c}0.3 \\
\text { Proposed }\end{array}$ & $\begin{array}{c}0.4 \\
\text { Kozat }\end{array}$ & $\begin{array}{c}0.4 \\
\text { Cover }\end{array}$ & $\begin{array}{c}0.4 \\
\text { Györfi }\end{array}$ & $\begin{array}{c}0.4 \\
\text { Proposed }\end{array}$ & $\begin{array}{c}0.5 \\
\text { Kozat }\end{array}$ & $\begin{array}{c}0.5 \\
\text { Cover }\end{array}$ & $\begin{array}{c}0.5 \\
\text { Györfi }\end{array}$ & $\begin{array}{c}0.5 \\
\text { Proposed }\end{array}$ \\
\hline \multicolumn{2}{|c|}{$\begin{array}{c}P \text {-value of JB test } \\
\text { Standard deviation }(\%)\end{array}$} & 0.603 & 0.716 & 0.074 & 0.066 & 0.603 & 0.712 & 0.059 & 0.056 & 0.603 & 0.707 & 0.063 & 0.067 \\
\hline \multicolumn{2}{|c|}{$\begin{array}{c}\text { Standard deviation (\%) } \\
\text { Mean (\%) }\end{array}$} & 12.1 & 12.3 & 13.9 & 14.3 & 12.1 & 12.1 & 13.7 & 13.8 & 12.1 & 12.0 & 13.3 & 13.2 \\
\hline \multirow{2}{*}{\multicolumn{2}{|c|}{$\begin{array}{c}\text { Difference of means }{ }^{\mathrm{a}}(\%) \\
P \text {-value of } t \text {-test }\end{array}$}} & $0.77^{* * *}$ & $0.95^{* * *}$ & $2.64^{* * *}$ & $3.04^{* * *}$ & $0.76^{* * *}$ & $0.80^{* * *}$ & $2.39^{* * *}$ & $2.52^{* * *}$ & $0.76^{* * *}$ & $0.65^{* * *}$ & $2.02^{* * *}$ & $1.91^{* * *}$ \\
\hline & & $6.0 \times 10^{-6}$ & $1.7 \times 10^{-7}$ & $1.7 \times 10^{-5}$ & $4.6 \times 10^{-7}$ & $7.3 \times 10^{-6}$ & $8.4 \times 10^{-6}$ & $1.2 \times 10^{-4}$ & $4.9 \times 10^{-5}$ & $8.8 \times 10^{-6}$ & $2.5 \times 10^{-4}$ & $1.2 \times 10^{-3}$ & $2.1 \times 10^{-3}$ \\
\hline
\end{tabular}

${ }^{\text {a }}$ Difference equals average annualised return of the corresponding method at each $c_{s}$ minus that of buy-and-hold $(\mathrm{B} \& \mathrm{H}) .{ }^{*} p<0.1 ;{ }^{* *} p<0.05 ;{ }^{* * *} p<0.01$.
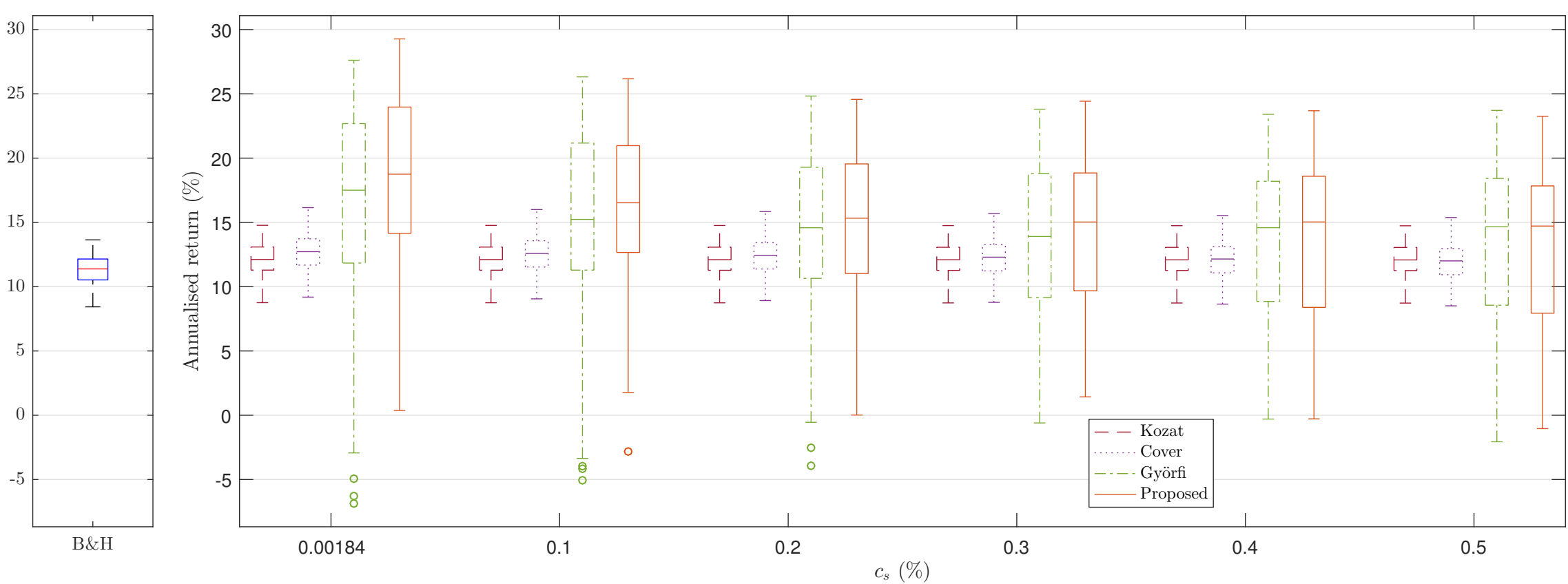

Figure 15. Box plot of annualised returns for comparison among Kozat, Cover, Györfi's, and proposed method when market liquidity is limited and $S_{0}=10^{4}\left(c_{p}=0\right)$. 
Table 5. Statistics of annualised returns for comparison among Kozat, Cover, Györfi's, and proposed method when market liquidity is limited and $S_{0}=10^{5}\left(c_{p}=0\right)$.

\begin{tabular}{|c|c|c|c|c|c|c|c|c|c|c|c|c|c|}
\hline$c_{s}(\%)$ & $\mathrm{B} \& \mathrm{H}$ & $\begin{array}{c}0.00184 \\
\text { Kozat }\end{array}$ & $\begin{array}{c}0.00184 \\
\text { Cover }\end{array}$ & $\begin{array}{c}0.00184 \\
\text { Györfi }\end{array}$ & $\begin{array}{c}0.00184 \\
\text { Proposed }\end{array}$ & $\begin{array}{c}0.1 \\
\text { Kozat }\end{array}$ & $\begin{array}{c}0.1 \\
\text { Cover }\end{array}$ & $\begin{array}{c}0.1 \\
\text { Györfi }\end{array}$ & $\begin{array}{c}0.1 \\
\text { Proposed }\end{array}$ & $\begin{array}{c}0.2 \\
\text { Kozat }\end{array}$ & $\begin{array}{c}0.2 \\
\text { Cover }\end{array}$ & $\begin{array}{c}0.2 \\
\text { Györfi }\end{array}$ & $\begin{array}{c}0.2 \\
\text { Proposed }\end{array}$ \\
\hline$P$-value of JB test & 0.419 & 0.603 & 0.731 & 0.008 & 0.027 & 0.601 & 0.726 & 0.009 & 0.015 & 0.602 & 0.722 & 0.021 & 0.165 \\
\hline Standard deviation (\%) & 1.15 & 1.19 & 1.33 & 8.51 & 6.79 & 1.19 & 1.33 & 7.83 & 5.71 & 1.19 & 1.32 & 5.92 & 5.36 \\
\hline Mean $(\%)$ & 11.3 & 12.1 & 12.7 & 15.3 & 17.6 & 12.1 & 12.6 & 14.2 & 15.9 & 12.1 & 12.4 & 14.3 & 14.7 \\
\hline Difference of means ${ }^{\mathrm{a}}(\%)$ & - & $0.79^{* * *}$ & $1.39^{* * *}$ & $3.95^{* * *}$ & $6.28^{* * *}$ & $0.79^{* * *}$ & $1.25^{* * *}$ & $2.93^{* * *}$ & $4.61^{* * *}$ & $0.78^{* *}$ & $1.10^{* * *}$ & $3.01^{* * *}$ & $3.40^{* * *}$ \\
\hline$P$-value of $t$-test & - & $3.4 \times 10^{-6}$ & $1.7 \times 10^{-13}$ & $1.2 \times 10^{-5}$ & $6.2 \times 10^{-15}$ & $4.1 \times 10^{-6}$ & $2.3 \times 10^{-11}$ & $3.4 \times 10^{-4}$ & $2.5 \times 10^{-12}$ & $5.0 \times 10^{-}$ & $2.5 \times 10^{-\mathrm{S}}$ & $2.3 \times 10^{-6}$ & $1.0 \times 10^{-8}$ \\
\hline \multicolumn{2}{|l|}{$c_{s}(\%)$} & $\begin{array}{c}0.3 \\
\text { Kozat }\end{array}$ & $\begin{array}{c}0.3 \\
\text { Cover }\end{array}$ & $\begin{array}{c}0.3 \\
\text { Györfi }\end{array}$ & $\begin{array}{c}0.3 \\
\text { Proposed }\end{array}$ & $\begin{array}{c}0.4 \\
\text { Kozat }\end{array}$ & $\begin{array}{c}0.4 \\
\text { Cover }\end{array}$ & $\begin{array}{c}0.4 \\
\text { Györfi }\end{array}$ & $\begin{array}{c}0.4 \\
\text { Proposed }\end{array}$ & $\begin{array}{c}0.5 \\
\text { Kozat }\end{array}$ & $\begin{array}{c}0.5 \\
\text { Cover }\end{array}$ & $\begin{array}{c}0.5 \\
\text { Györfi }\end{array}$ & $\begin{array}{c}0.5 \\
\text { Proposed }\end{array}$ \\
\hline \multicolumn{2}{|c|}{$\begin{array}{c}P \text {-value of JB test } \\
\text { Standard deviation }(\%)\end{array}$} & 0.603 & 0.717 & 0.073 & 0.059 & 0.603 & 0.711 & 0.059 & 0.057 & 0.605 & 0.706 & 0.063 & 0.077 \\
\hline \multicolumn{2}{|c|}{$\begin{array}{c}\text { Standard deviation (\%) } \\
\text { Mean (\%) }\end{array}$} & 12.1 & 12.3 & 13.9 & 13.9 & 12.1 & 12.1 & 13.7 & 13.4 & 12.1 & 12.0 & 13.3 & 12.9 \\
\hline \multirow{2}{*}{\multicolumn{2}{|c|}{$\begin{array}{c}\text { Difference of means }{ }^{\mathrm{a}}(\%) \\
P \text {-value of } t \text {-test }\end{array}$}} & $0.77^{* * *}$ & $0.95^{* * *}$ & $2.57^{* * *}$ & $2.58^{* * *}$ & $0.76^{* * *}$ & $0.80^{* * *}$ & $2.35^{* * *}$ & $2.14^{* * *}$ & $0.76^{* * *}$ & $0.65^{* * *}$ & $1.99^{* * *}$ & $1.64^{* * *}$ \\
\hline & & $6.0 \times 10^{-6}$ & $1.8 \times 10^{-7}$ & $2.6 \times 10^{-5}$ & $1.0 \times 10^{-5}$ & $7.3 \times 10^{-6}$ & $8.8 \times 10^{-6}$ & $1.6 \times 10^{-4}$ & $4.5 \times 10^{-4}$ & $8.8 \times 10^{-6}$ & $2.6 \times 10^{-4}$ & $1.4 \times 10^{-3}$ & $6.7 \times 10^{-3}$ \\
\hline
\end{tabular}

${ }^{a}$ Difference equals average annualised return of the corresponding method at each $c_{s}$ minus that of buy-and-hold $(\mathrm{B} \& \mathrm{H}) .{ }^{*} p<0.1 ;{ }^{* *} p<0.05 ;{ }^{* * *} p<0.01$.
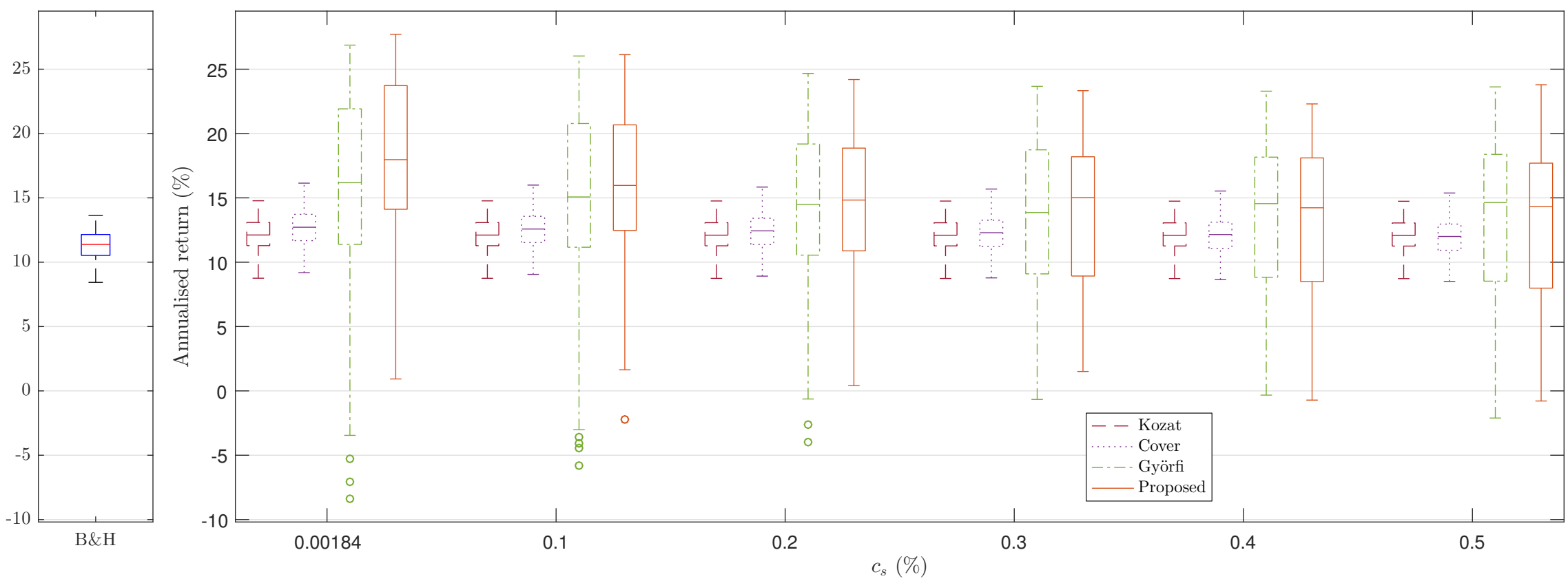

Figure 16. Box plot of annualised returns for comparison among Kozat, Cover, Györfi, and proposed method when market liquidity is limited and $S_{0}=10^{5}\left(c_{p}=0\right)$. 
Table 6. Mean difference of annualised returns between Györfi's and proposed method when $S_{0}=10^{4}\left(c_{p}=0\right)$.

\begin{tabular}{ccccccc}
\hline$c_{s}(\%)$ & 0.00184 & 0.1 & 0.2 & 0.3 & 0.4 & 0.5 \\
\hline Difference of means $^{\mathrm{a}}(\%)$ & $1.94^{*}$ & $1.62^{*}$ & 0.60 & 0.40 & 0.12 & -0.12 \\
$P$-value of $t$-test & 0.080 & 0.099 & 0.457 & 0.614 & 0.882 & 0.889 \\
\hline
\end{tabular}

${ }^{\text {a }}$ Difference equals average annualised return of the proposed method minus that of Györfi. ${ }^{*} p<0.1 ;{ }^{* *} p<0.05 ;{ }^{* *} p<0.01$.

Table 7. Mean difference of annualised returns between Györfi's and proposed method when $S_{0}=10^{5}\left(c_{p}=0\right)$.

\begin{tabular}{ccccccc}
\hline$c_{s}(\%)$ & 0.00184 & 0.1 & 0.2 & 0.3 & 0.4 & 0.5 \\
\hline Difference of means $^{\mathrm{a}}(\%)$ & $2.33^{* *}$ & $1.68^{*}$ & 0.39 & 0.01 & -0.21 & -0.35 \\
$P$-value of $t$-test & 0.034 & 0.085 & 0.626 & 0.991 & 0.797 & 0.673 \\
\hline
\end{tabular}

${ }^{a}$ Difference equals average annualised return of the proposed method minus that of Györfi. ${ }^{*} p<0.1 ;{ }^{* *} p<0.05 ;{ }^{* *} p<0.01$.

on initial wealth $S_{0}$ because TCF $w_{n}$ in (29) is a function of $S_{n}$ : i.e. the greater $S_{0}$; the greater MICs, which in turn causes less performance of OPS compared to Section 8.1. However, only the proposed method takes into account the LOB data as well as $c_{p}, c_{s}$ when calculating $\boldsymbol{b}_{n+1}$.

8.2.1. When initial wealth $S_{0}$ is small $\left(S_{0}=1^{4}\right)$. When initial wealth $S_{0}$ is as small as USD 10k, the performance deterioration of the Györfi's method is severe when comparing between Figure 13 and 14 (or between Figure 12 and 15). In addition, the performance difference between the Györfi's and proposed method is marginally statistically significant when $c_{s} \leq 0.1 \%$ as shown in Table 6, which means that the proposed method is not effective compared to the Györfi's method for small-sized funds with high proportional TCs. Moreover, the $p$-value tends to increase as $c_{s}$ increases, which proves the low effectiveness of the proposed method with high proportional TCs. This was foreseen in Section 5.3: the proportion of MICs in TCs decreases as $c_{p}$ or $c_{s}$ increases, which in turn makes the proposed method less different from the Györfi's method.

8.2.2. When initial wealth $\boldsymbol{S}_{\mathbf{0}}$ is large $\left(\boldsymbol{S}_{\mathbf{0}}=\mathbf{1 0}^{\mathbf{5}}\right)$. When $S_{0}$ is as large as USD 100k (a hundred thousand US dollars is not a large fund size but relatively large for the Györfi's and proposed method in terms of MICs), the performance deterioration of the Györfi's method is higher than the proposed method when comparing between Figure 14 and 17 (or between Figure 15 and 16). To be specific, the proposed method is fundamentally superior to the Györfi method when $c_{s}=0.00184 \%$ and $S_{0}=10^{5}$, while it is marginally fundamentally superior to the Györfi method when $c_{s}=0.00184 \%$ and $S_{0}=10^{4}$. This means that the proposed method is more effective than the Györfi method for large-sized funds and low TC rates (i.e. small value of $\frac{c_{p}+c_{s}}{1+c_{p}}$ ).

The performance of the Kozat's method and the Cover's method does not change even when $S_{0}$ increases from USD $10^{4}$ to $10^{5}$ as shown in Figure 14 and 17. This is for the following two reasons: i) $\boldsymbol{b}_{n+1}$ is independent of $S_{n}$ in the case of the Kozat's method and the Cover's method. ii) The Kozat's method and the Cover's method trade much less than the Györfi's and the proposed method. I.e. the market order size $q_{n}^{(j)}$ in (29) of the Kozat's method and the Cover's method is much less than that of the Györfi's and the proposed method (this will be shown in Figure 20); hence, $\bar{p}\left(q_{n}^{(j)}\right)$ in (29) is replaced with either $P_{1}$ or $P_{-1}$ (i.e. the best ask or bid price) for both $S_{0}=10^{4}$ and $S_{0}=10^{5}$, which results in the same TCF and same performance for each $c_{s}$ between $S_{0}=10^{4}$ and $S_{0}=10^{5}$.

\subsection{Graphical comparisons}

Figure 18 shows the gross wealth $S_{n}$ of a portfolio of five stocks when market liquidity is limited, and when initial wealth $S_{0}$ is USD 100k, where Figure 18(b) is the magnified plot of the beginning 


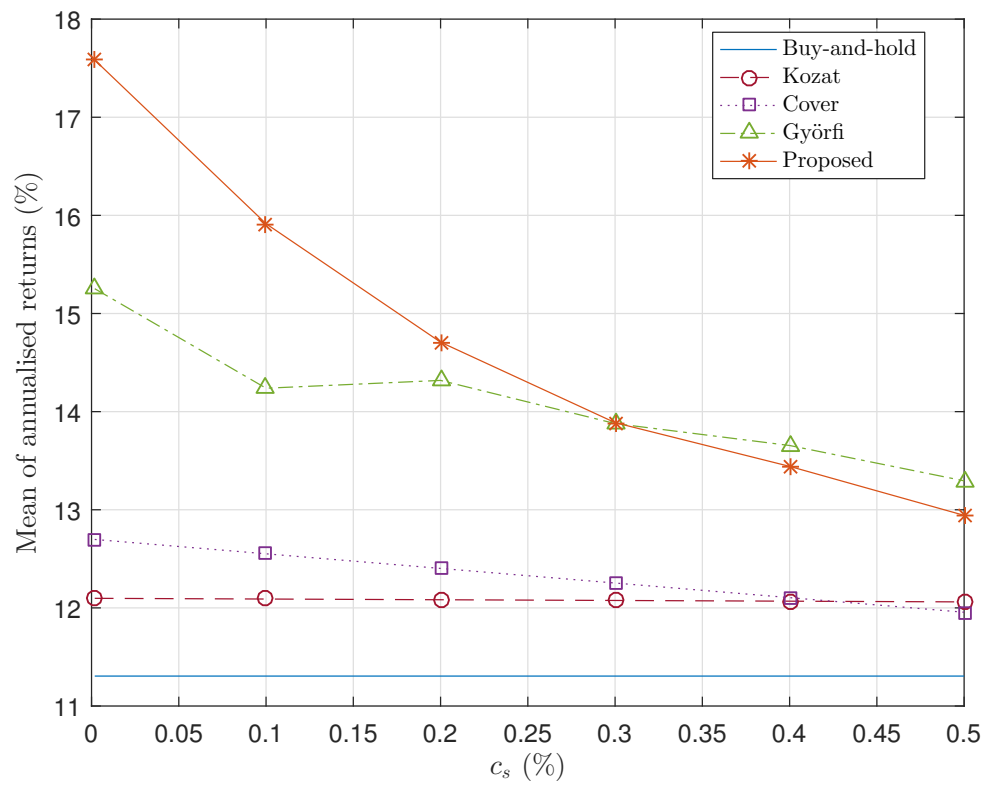

Figure 17. Mean of annualised returns for comparison among Kozat, Cover, Györfi, and proposed method when market liquidity is limited and $S_{0}=10^{5}\left(c_{p}=0\right)$.

part of Figure 18(a). All the OPS methods made huge losses at the beginning part due to the financial crisis of 2008, but they have been converted to profits by the bull NASDAQ stock market for seven years since 2009 .

Figure 19 shows the proportion of portfolio (i.e. the portfolio vector $\boldsymbol{b}_{n}$ ) that made the gross wealth in Figure 18(b), in the form of area plots. The portfolio vector of B\&H changes over time, as shown in Figure 19(a), as the prices of assets change over time, and Figure 19(b) is similar to Figure 19(a) because the Kozat's method tries to minimize TCs. On the one hand, the Cover's method made an almost constant portfolio vector over time as shown in Figure 19(c), but on the other hand, the Györfi's and the proposed method generated abrupt changes of the portfolio vector over time, as shown in Figure 19(d) and 19(e). Meanwhile, Figure 19(e) shows fewer spikes than Figure 19(d) as the proposed method impedes the rapid portfolio changes: i.e. it considers MICs and makes a decision that a small change of the portfolio vector is more profitable than a large change.

Figure 20 shows market orders $q_{n}^{(j)}, \forall j=\{1,2, \ldots, 5\}$ that made the gross wealth in Figure 18(b), calculated from (26). The amplitude of the market order varies among the OPS methods. In particular, Figure 20(d), by the proposed method, shows smaller amplitude than Figure 20(c), by the Györfi's method; the amplitude difference between Figure 20(d) and 20(c) corresponds to the difference between Figure 19(e) and 19(d).

Figure 21 shows TCs (including MICs) by the marker orders in Figure 20 and confirms that the more TCs are charged as the greater magnitude of market order is generated, where TC including MIC at the end of the $n$-th day is calculated as

$$
C_{n}=\left(1-w_{n}\right) S_{n}
$$

from (16) and (18). Both the Györfi's and the proposed method lead to zero TCs (i.e. $C_{n}=0$, which means no trading at the end of the $n$-th day) on some days as shown in Figure 21(b), whereas the Kozat's method and the Cover's method do not, as shown in Figure 21(a). The zero TCs of the Györfi's and proposed method are caused by the two possibilities: no matching (i.e. $J_{n}=\varnothing$ in (12) for all $l$ ) and the dominance of TCs (i.e. $\boldsymbol{b}_{n+1}^{\star}$ in (30) is the solution of (11) for all $l$ ). 


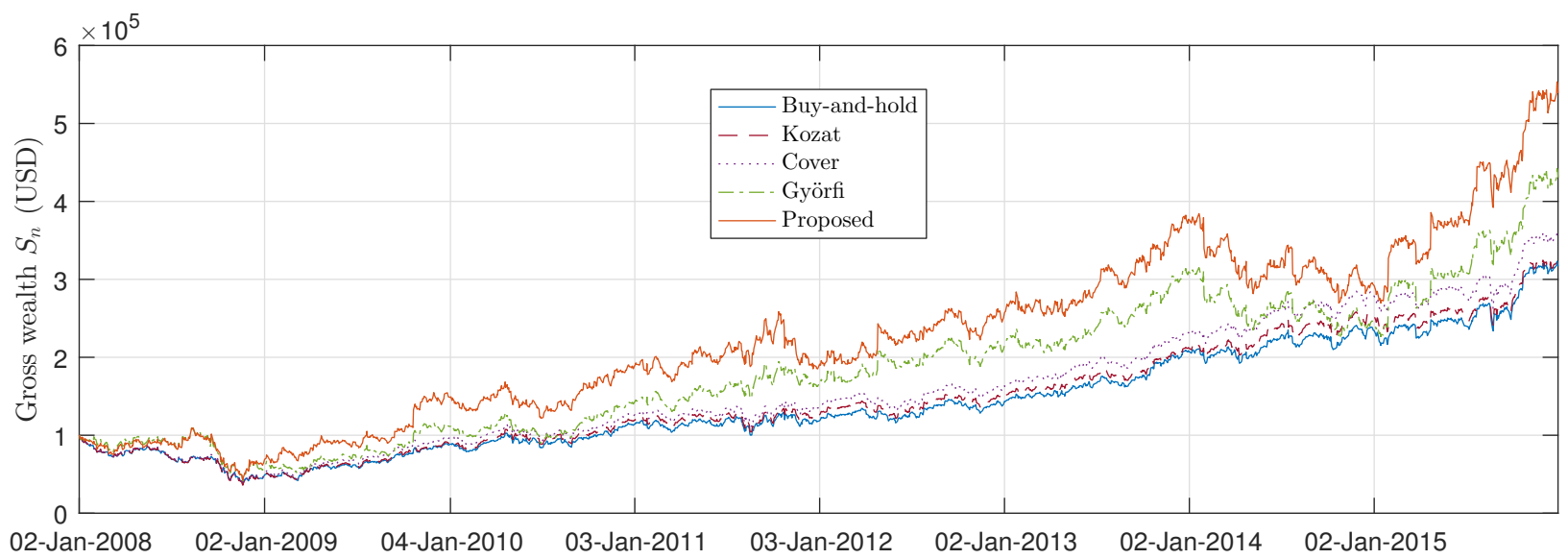

(a) Between 2 Jan 2008 and 31 Dec 2015 (annualised return is buy-and-hold 15.5\%; Kozat 15.7\%; Cover 17.1\%; Györfi 20.1\%; proposed method $23.4 \%$ ).

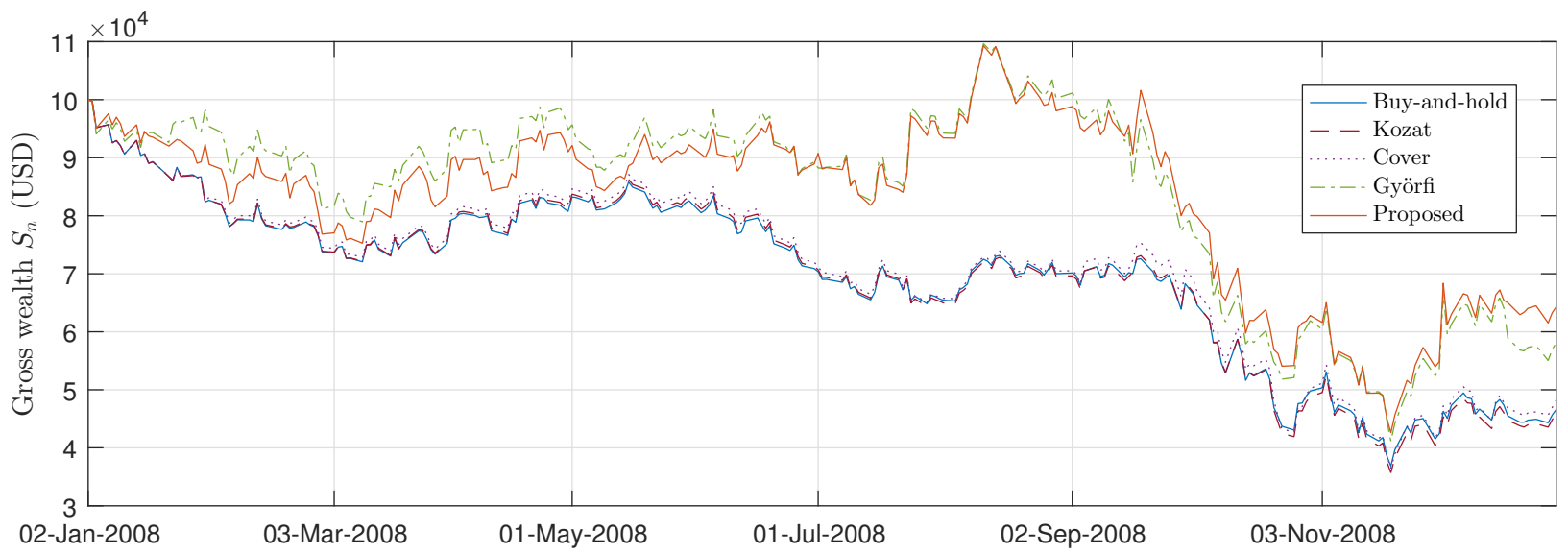

(b) Between 2 Jan 2008 and 31 Dec 2008.

Figure 18. Gross wealth over time when the portfolio consists of AMZN, CDNS, CTAS, MSFT, and SNDK $\left(S_{0}=10^{5}\right.$, $\left.c_{p}=0, c_{s}=0.00184 \%\right)$.

Table 8. Sample mean and standard deviation of computation time $(d=30)$.

\begin{tabular}{rcccrr}
\hline & Kozat & Cover & Györfi & Proposed $\left(S_{0}=10^{4}\right)$ & Proposed $\left(S_{0}=10^{5}\right)$ \\
\hline Time (seconds) & $0.20 \pm 0.02$ & $0.03 \pm 0.00$ & $0.84 \pm 1.31$ & $0.97 \pm 0.87$ & $1.79 \pm 1.22$ \\
\hline
\end{tabular}

\subsection{Computation time}

Table 8 shows the computation time to calculate $\boldsymbol{b}_{n+1}$ for the simulations in Section 8.2 by using AMD Opteron ${ }^{\mathrm{TM}} 6376 \mathrm{CPU}$ and MATLAB R2011b. The computation time to calculate $\boldsymbol{b}_{n+1}$ depends on $n$ (i.e. the greater $n$; the longer computation time) in the case of the Kozat's, Györfi's, and proposed method. Thus, only the worst case, $n=2013,{ }^{1}$ was measured for all the OPS methods. It takes longer for the proposed method to calculate $\boldsymbol{b}_{n+1}$ by the additional time to calculate MICs than the Györfi's method. In addition, the computation time of the proposed method increases as the initial wealth $S_{0}$ increases. This is because the larger wealth causes the heavier burden to calculate $\bar{p}(q)$ in (14) by additionally using LOB data in higher absolute levels.

\footnotetext{
130 Dec $2015(n=2013)$, not 31 Dec $2015(n=2014)$, was the last day to calculate $\boldsymbol{b}_{n+1}$, where 2 Jan 2008 corresponds to $n=0$ (i.e. time 0; see Figure 1).
} 


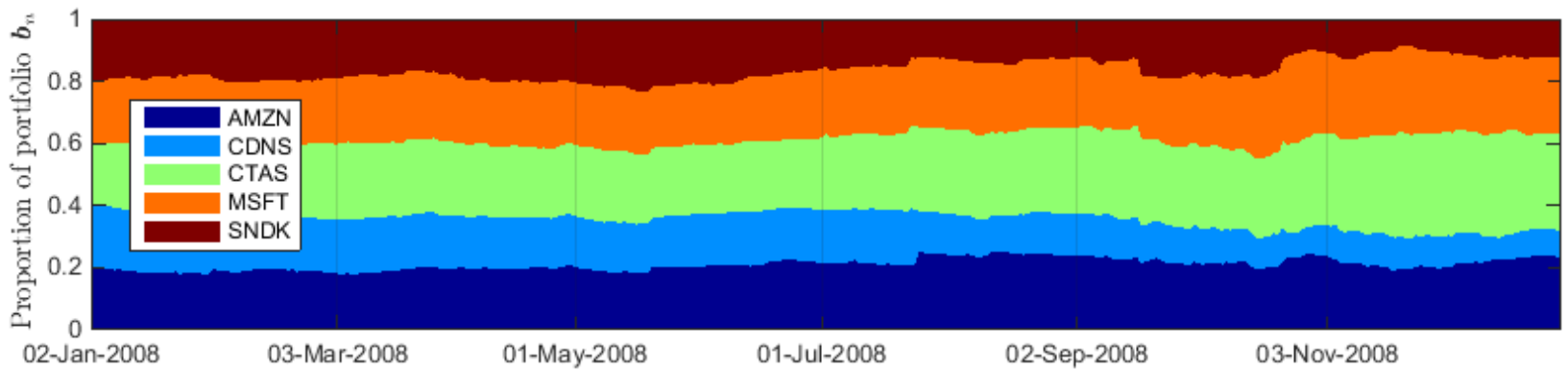

(a) Buy-and-hold.

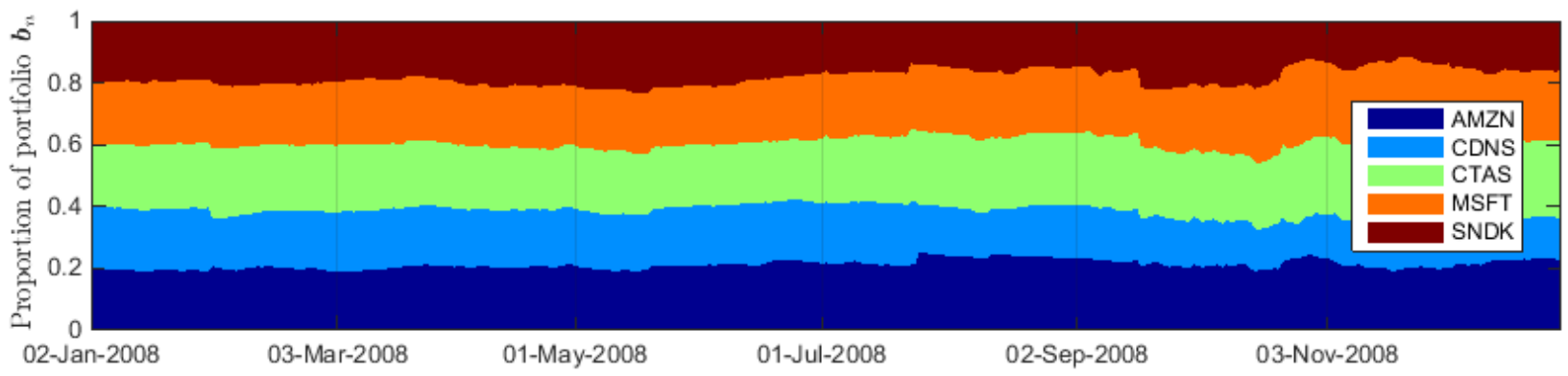

(b) Kozat.

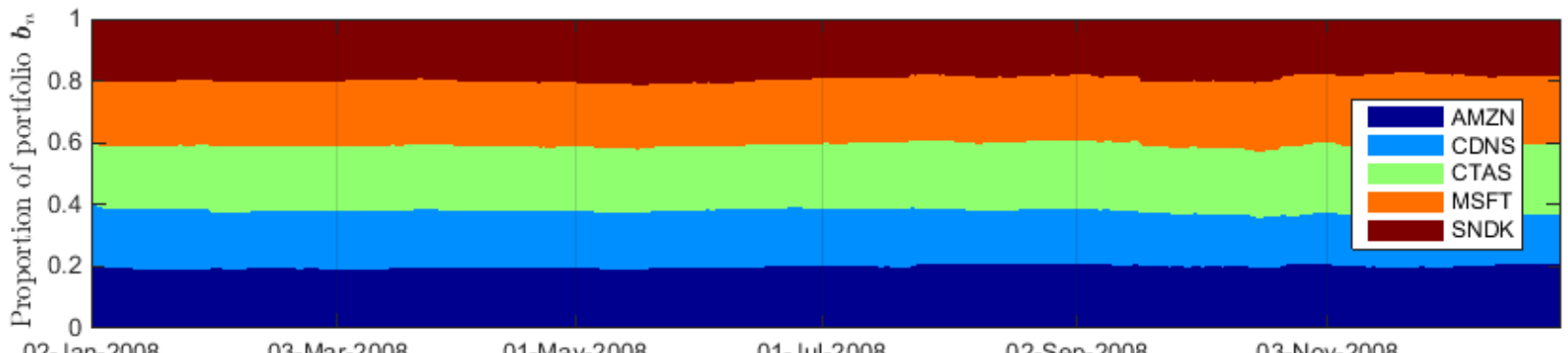

02-Jan-2008 03-Mar-2008 01-May-2008 01-Jul-2008 $\quad$ 02-Sep-2008

(c) Cover

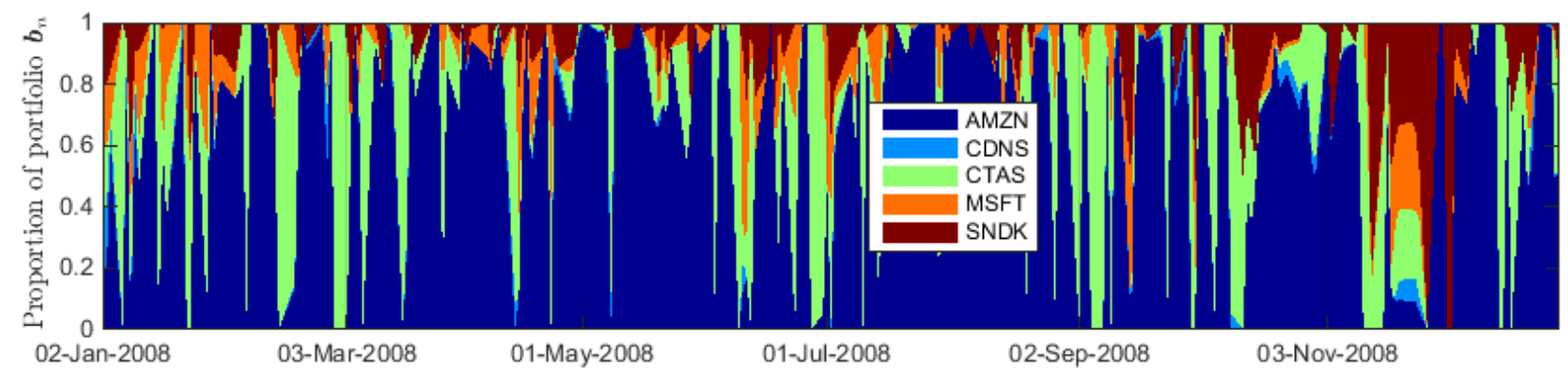

(d) Györfi.

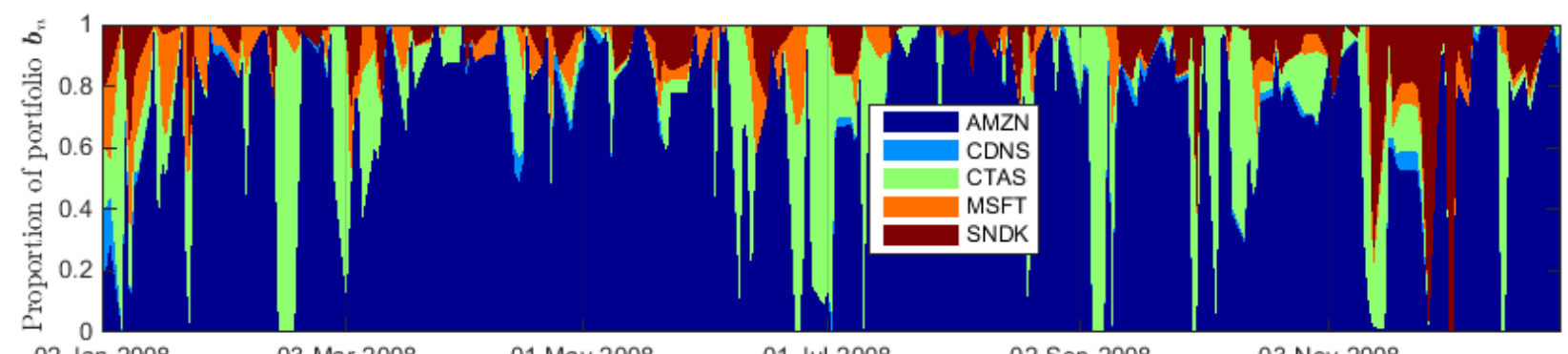

(e) Proposed method.

Figure 19. Proportion of portfolio over time between 2 Jan 2008 and 31 Dec $2008\left(S_{0}=10^{5}, c_{p}=0, c_{s}=0.00184 \%\right)$. 


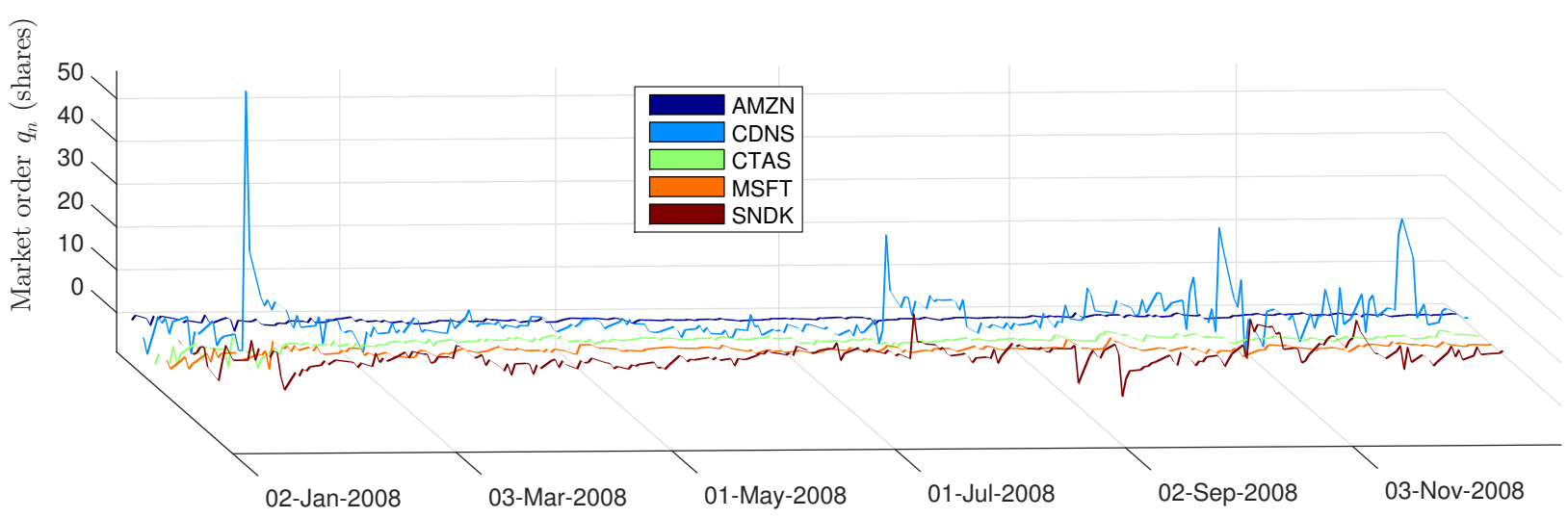

(a) Kozat.

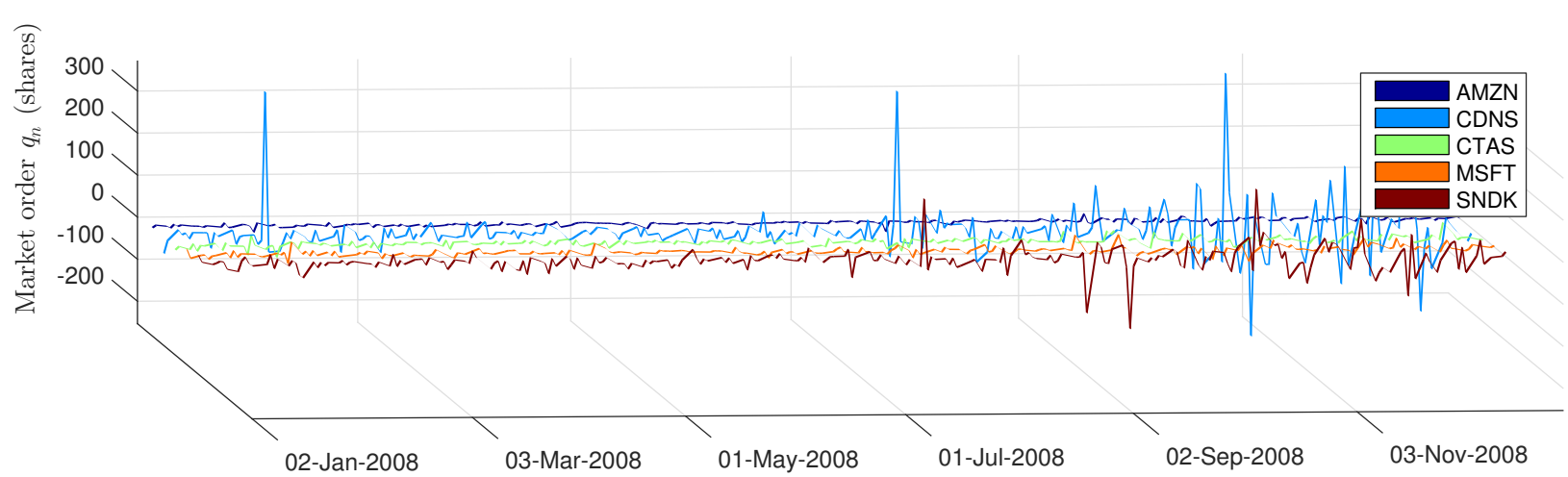

(b) Cover.

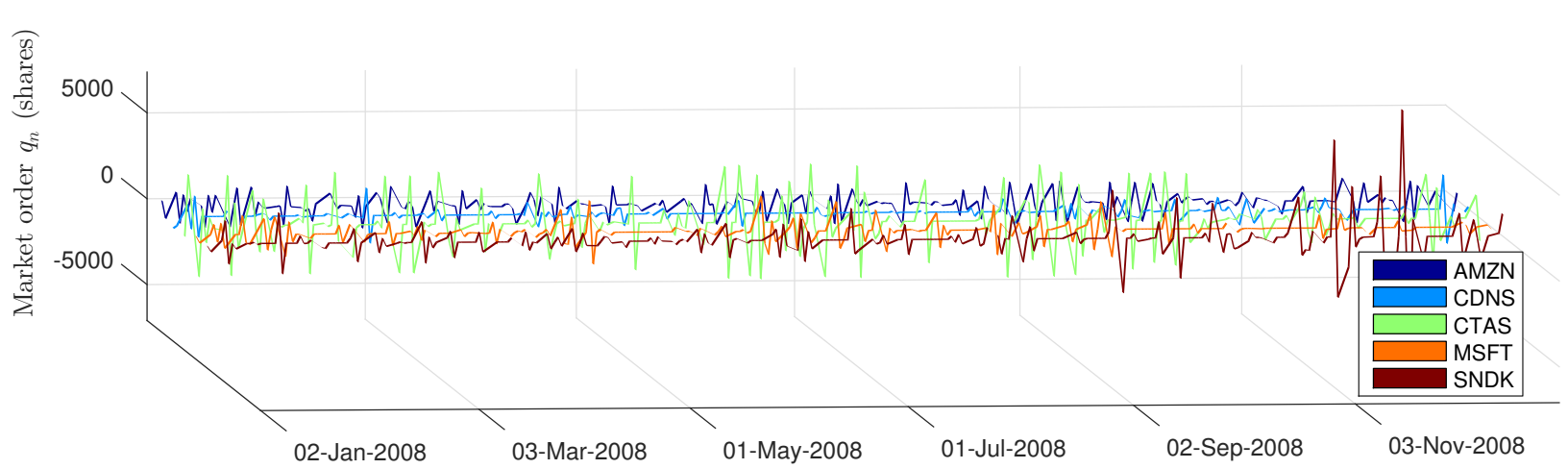

(c) Györfi.

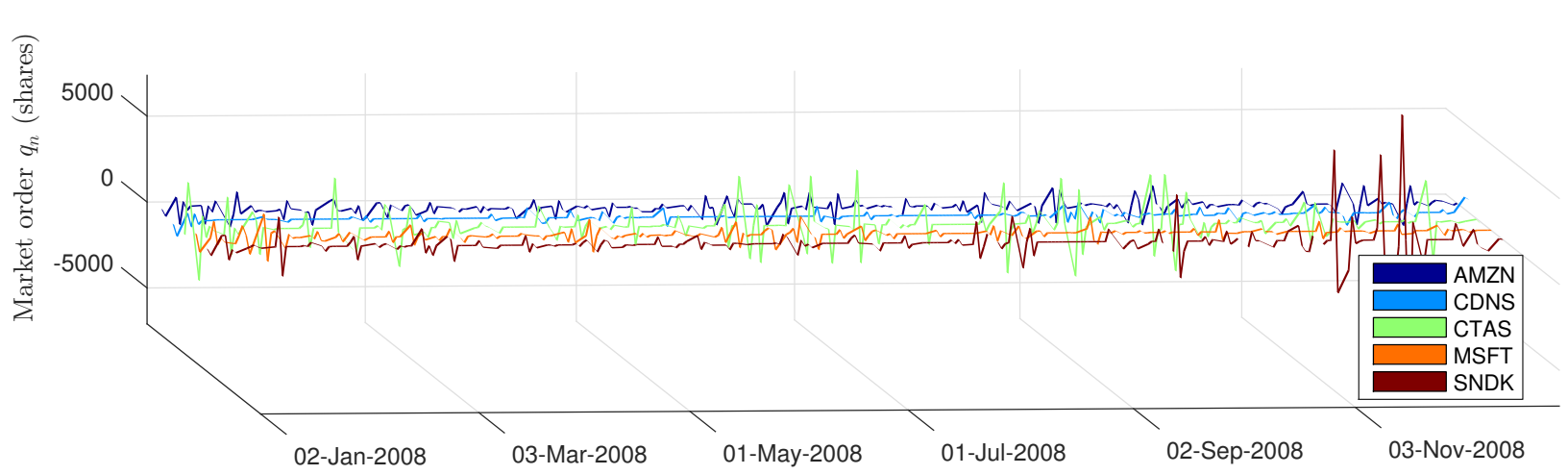

(d) Proposed method.

Figure 20. Market order over time between 2 Jan 2008 and 31 Dec $2008\left(S_{0}=10^{5}, c_{p}=0, c_{s}=0.00184 \%\right)$. Positive

(negative) values of market order indicate buying (selling) stocks. 


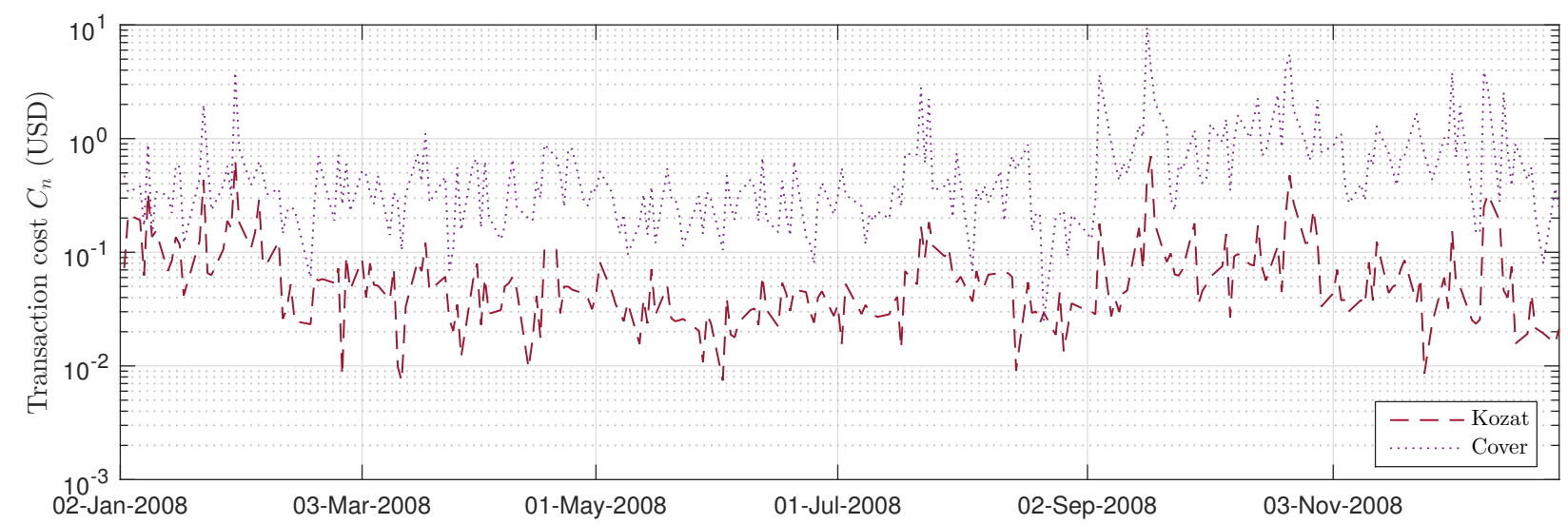

(a) Kozat and Cover.

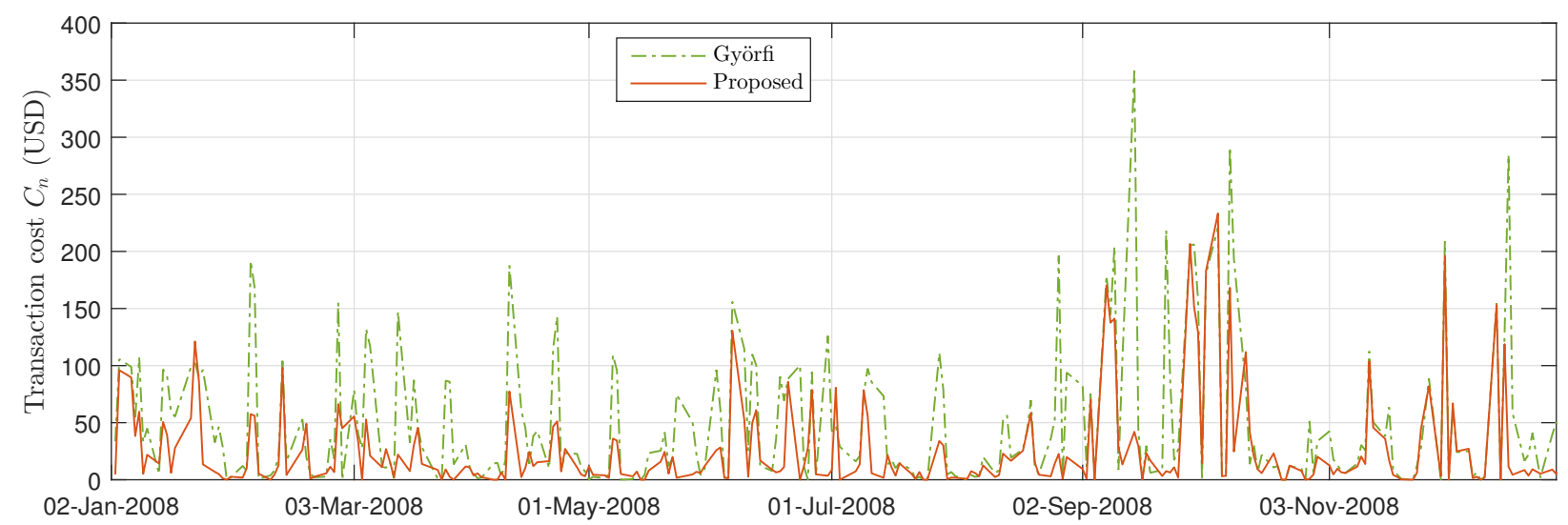

(b) Györfi's and proposed method.

Figure 21. Transaction costs including market impact costs over time between 2 Jan 2008 and 31 Dec $2008\left(S_{0}=10^{5}\right.$, $\left.c_{p}=0, c_{s}=0.00184 \%\right)$.

\section{Conclusion}

In this paper, we have developed a new practical TCF model of OPS by considering not only the proportional transaction costs but liquidity risks which is quantified by LOB data as well. The proposed OPS is much more practical compared to previous TCF models (Kozat and Singer 2011, Cover 1991, Györfi and Vajda 2008) as it relaxes the unlimited liquidity assumption. Based on reslults of the backtesting, the proposed OPS method shows its superiority to the existing method (Györfi and Vajda 2008) for large-sized funds with low TC rates.

A tradeoff between OPS performance and fund size has been noted in our OPS method by using the historical LOB data of NASDAQ 100 Index Components, in Section 8.2. For future research, it would be worthwhile to focus on optimal splitting of a large market order into a number of smaller consecutive marker orders to reduce overall MICs and make OPS strategies practical even for large-sized funds.

\section{Acknowledgements}

The financial support of EUR 4,798 from the Department of Economics at the University of Glasgow to purchase the LOBSTER data is acknowledged. In addition, the High Performance Compute Cluster of IT Services at the University of Glasgow reduced the total time required to obtain the MC simulation results in Section 8. 


\section{References}

Alfonsi, A., Fruth, A. and Schied, A., Constrained portfolio liquidation in a limit order book model. In Proceedings of the Advances in mathematics of finance, Vol. 83 of Banach Center publications, pp. 9--25, 2008.

Bauwens, L., Pohlmeier, W. and Veredas, D., High frequency financial econometrics: Recent developments, 2007, Physica-Verlag.

Bean, A.J. and Singer, A.C., Universal switching and side information portfolios under transaction costs using factor graphs. IEEE Journal of Selected Topics in Signal Processing, 2012, 6, 351--365.

Ben-Tal, A., Margalit, T. and Nemirovski, A., Robust modeling of multi-stage portfolio problems. In High performance optimization, pp. 303--328, 2000, Springer.

Blum, A. and Kalai, A., Universal portfolios with and without transaction costs. Machine Learning, 1999, 35, 193--205.

Borodin, A. and El-Yaniv, R., Online computation and competitive analysis, 1998, Cambridge University Press.

Borodin, A., El-Yaniv, R. and Gogan, V., Can we learn to beat the best stock. Journal of Artificial Intelligence Research, 2004, pp. 579--594.

Boyd, S. and Vandenberghe, L., Convex optimization, 2004, Cambridge University Press.

Brown, D.B. and Smith, J.E., Dynamic portfolio optimization with transaction costs: Heuristics and dual bounds. Management Science, 2011, 57, 1752--1770.

Bussieck, M.R. and Vigerske, S., MINLP solver software. In Wiley Encyclopedia of Operations Research and Management Science, 2011, Wiley.

Cover, T.M., Universal portfolios. Mathematical finance, 1991, 1, 1--29.

Cover, T.M. and Ordentlich, E., Universal portfolios with side information. IEEE Transactions on Information Theory, 1996, 42, 348--363.

Cover, T.M. and Thomas, J.A., Elements of information theory (2nd edn), 2006, Wiley.

Damodaran, A., Investment philosophies: Successful strategies and the investors who made them work (2nd edn), 2012, Wiley.

Dantzig, G.B. and Infanger, G., Multi-stage stochastic linear programs for portfolio optimization. Annals of Operations Research, 1993, 45, 59--76.

Das, P., Online convex optimization and its application to online portfolio selection. $\mathrm{PhD}$ thesis, University of Minnesota, 2014.

Das, P., Johnson, N. and Banerjee, A., Online lazy updates for portfolio selection with transaction costs. In Proceedings of the Twenty-Seventh AAAI Conference on Artificial Intelligence, pp. 202--208, 2013.

Das, P., Johnson, N. and Banerjee, A., Online portfolio selection with group sparsity. In Proceedings of the Twenty-Eighth AAAI Conference on Artificial Intelligence, pp. 1185--1191, 2014.

DeMiguel, V., Nogales, F.J. and Uppal, R., Stock return serial dependence and out-of-sample portfolio performance. Review of Financial Studies, 2014, 27, 1031--1073.

Ferreira, M.A. and Santa-Clara, P., Forecasting stock market returns: The sum of the parts is more than the whole. Journal of Financial Economics, 2011, 100, 514--537.

Freitas, F.D., De Souza, A.F. and de Almeida, A.R., Prediction-based portfolio optimization model using neural networks. Neurocomputing, 2009, 72, 2155--2170.

Gaivoronski, A.A. and Pflug, G., Value-at-risk in portfolio optimization: Properties and computational approach. Journal of Risk, 2005, 7, 1--31.

Gârleanu, N. and Pedersen, L.H., Dynamic trading with predictable returns and transaction costs. Journal of Finance, 2013, 68, 2309--2340.

Györfi, L., Lugosi, G. and Udina, F., Nonparametric kernel-based sequential investment strategies. Mathematical Finance, 2006, 16, 337--357.

Györfi, L., Ottucsák, G. and Urbán, A., Empirical log-optimal portfolio selections: A survey. In Machine learning for financial engineering, pp. 81--118, 2012, Imperial College Press.

Györfi, L., Udina, F. and Walk, H., Experiments on universal portfolio selection using data from real markets. Technical report, 2008a.

Györfi, L., Udina, F. and Walk, H., Nonparametric nearest neighbor based empirical portfolio selection strategies. Statistics \& Decisions, 2008b, 26, 145--157.

Györfi, L., Urbán, A. and Vajda, I., Kernel-based semi-log-optimal empirical portfolio selection strategies. International Journal of Theoretical and Applied Finance, 2007, 10, 505--516. 
Györfi, L. and Vajda, I., Growth optimal investment with transaction costs. In Algorithmic Learning Theory, pp. 108--122, 2008, Springer.

Györfi, L. and Walk, H., Empirical portfolio selection strategies with proportional transaction costs. IEEE Transactions on Information Theory, 2012, 58, 6320--6331.

Horváth, M. and Urbán, A., Growth optimal portfolio selection with short selling and leverage. In Machine learning for financial engineering, pp. 153--178, 2012, Imperial College Press.

Huang, R. and Polak, T., LOBSTER: Limit order book reconstruction system. Working paper, available at http://ssrn. com/abstract=1977207, 2011.

Ishijima, H., Numerical methods for universal portfolios. In Proceedings of the Quantitative Methods in Finance Conference, 2001.

Kozat, S.S. and Singer, A.C., Universal semiconstant rebalanced portfolios. Mathematical Finance, 2011, 21, 293--311.

Levy, H. and Post, T., Investments, 2005, Pearson Education.

Li, B. and Hoi, S.C., Online portfolio selection: A survey. ACM Computing Surveys, 2014, 46, 35.

Li, D. and Ng, W.L., Optimal dynamic portfolio selection: Multiperiod mean-variance formulation. Mathematical Finance, 2000, 10, 387--406.

Luenberger, D.G., Investment science, 1998, Oxford University Press.

Markowitz, H.M., Portfolio selection: efficient diversification of investments, 1959, Yale University Press.

Matheson, T., Taxing financial transactions: Issues and evidence. Working paper 11/54, International Monetary Fund, 2011.

Olsson, R., Portfolio management under transaction costs: Model development and Swedish evidence. PhD thesis, Umeå University, 2005.

Ormos, M. and Urbán, A., Performance analysis of log-optimal portfolio strategies with transaction costs. Quantitative Finance, 2013, 13, 1587--1597.

Otranto, E., Asset allocation using flexible dynamic correlation models with regime switching. Quantitative Finance, 2010, 10, 325--338.

Palczewski, J., Poulsen, R., Schenk-Hoppé, K.R. and Wang, H., Dynamic portfolio optimization with transaction costs and state-dependent drift. European Journal of Operational Research, 2015, 243, 921--931.

Pristas, G., Limit order book dynamics and asset liquidity. PhD thesis, University of Zurich, 2007.

Ruxton, G.D., The unequal variance t-test is an underused alternative to Student's t-test and the Mann-Whitney U test. Behavioral Ecology, 2006, 17, 688--690.

Simchi-Levi, D., Chen, X. and Bramel, J., The logic of logistics: Theory, algorithms, and applications for logistics management (3rd edn), 2014, Springer.

Simon, D., Evolutionary optimization algorithms, 2013, Wiley.

Skovlund, E. and Fenstad, G.U., Should we always choose a nonparametric test when comparing two apparently nonnormal distributions?. Journal of Clinical Epidemiology, 2001, 54, 86--92.

Steinbach, M.C., Markowitz revisited: Mean-variance models in financial portfolio analysis. SIAM review, 2001, 43, 31--85.

Sydsæter, K., Strøm, A. and Berck, P., Economists' mathematical manual (4th edn), 2010, Springer.

Tunc, S., Donmez, M.A. and Kozat, S.S., Optimal investment under transaction costs: A threshold rebalanced portfolio approach. IEEE Transactions on Signal Processing, 2013, 61, 3129--3142.

\section{Appendix A: Difference between (Cover 1991) and (Blum and Kalai 1999)}

\section{Appendix B: Concavity of Equation (4)}

Let us check whether $\sum_{i \in J_{n}} \ln \left\langle\boldsymbol{b}, \boldsymbol{x}_{i+1}\right\rangle$ in (4) is a concave function of $\boldsymbol{b} \in \Delta^{d-1}$ or not. The first-order partial derivative of $\ln \left\langle\boldsymbol{b}, \boldsymbol{x}_{i+1}\right\rangle$ with respect to $b^{(j)}$ (Figure 8 shows the direction of the partial derivative when $d=3$ ) is

$$
\frac{\partial \ln \left\langle\boldsymbol{b}, \boldsymbol{x}_{i+1}\right\rangle}{\partial b^{(j)}}=\frac{x_{i+1}^{(j)}-x_{i+1}^{\left(j^{\prime}\right)}}{\left\langle\boldsymbol{b}, \boldsymbol{x}_{i+1}\right\rangle},
$$




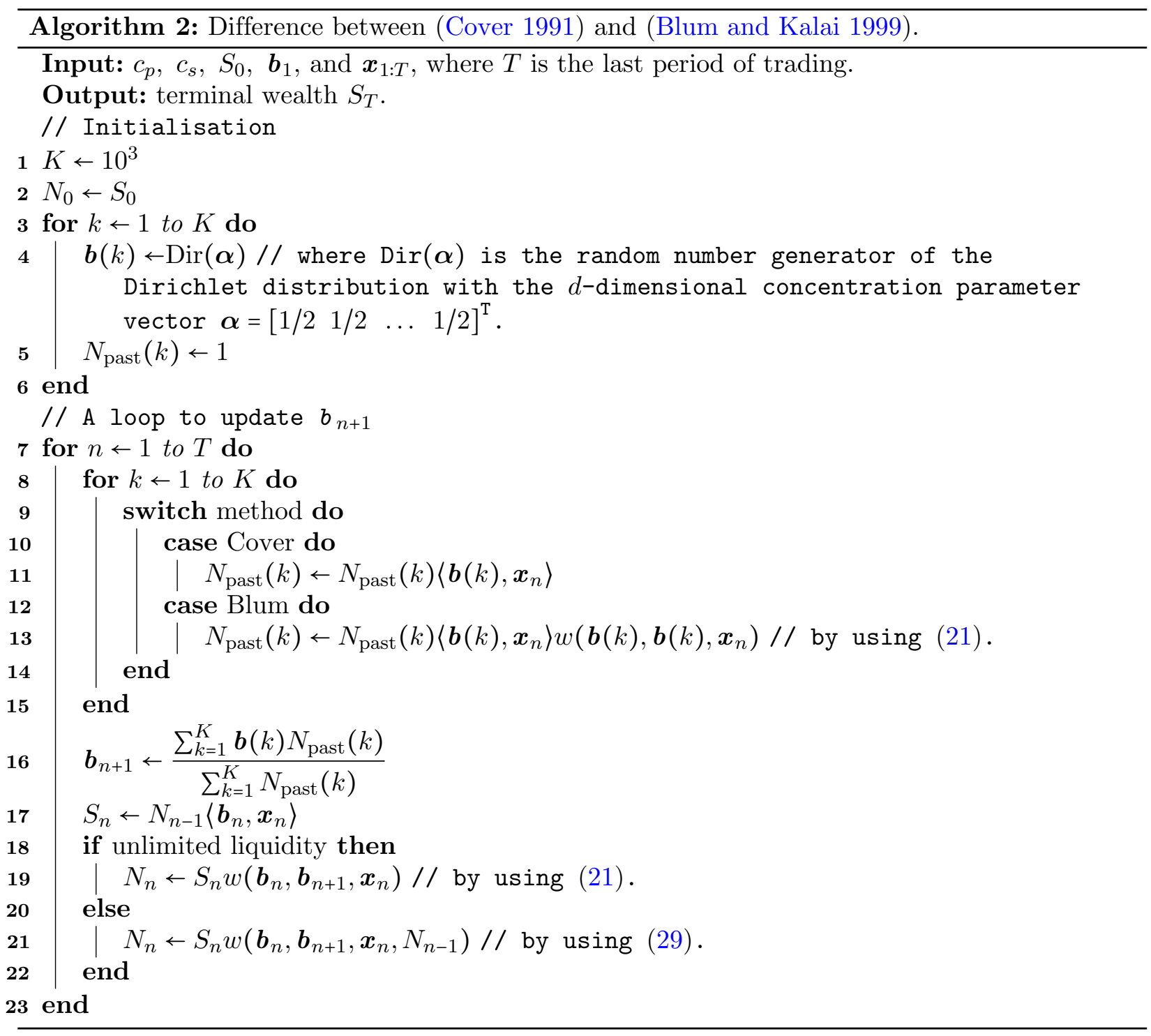

where $j^{\prime} \stackrel{\text { def }}{=}\left\{\begin{array}{cl}d, & \text { if } j=1 \\ j-1, & \text { otherwise }\end{array}\right.$. The second-order partial derivative is

$$
\frac{\partial^{2} \ln \left\langle\boldsymbol{b}, \boldsymbol{x}_{i+1}\right\rangle}{\left(\partial b^{(j)}\right)^{2}}=-\frac{\left(x_{i+1}^{(j)}-x_{i+1}^{\left(j^{\prime}\right)}\right)^{2}}{\left\langle\boldsymbol{b}, \boldsymbol{x}_{i+1}\right\rangle^{2}}
$$

and the second-order mixed partial derivative is

$$
\frac{\partial^{2} \ln \left\langle\boldsymbol{b}, \boldsymbol{x}_{i+1}\right\rangle}{\partial b^{(j)} \partial b^{(k)}}=-\frac{\left(x_{i+1}^{(j)}-x_{i+1}^{\left(j^{\prime}\right)}\right)\left(x_{i+1}^{(k)}-x_{i+1}^{\left(k^{\prime}\right)}\right)}{\left\langle\boldsymbol{b}, \boldsymbol{x}_{i+1}\right\rangle^{2}},
$$

where $k^{\prime} \stackrel{\text { def }}{=}\left\{\begin{array}{cl}d, & \text { if } k=1 \\ k-1, & \text { otherwise }\end{array}\right.$, and $j \neq k$.

Theorem B.1 A (twice differentiable) function $\mathbb{R}^{d} \rightarrow \mathbb{R}$ is concave (convex) if and only if its 
Hessian matrix is negative (positive) semidefinite.

The Hessian matrix of $\ln \left\langle\boldsymbol{b}, \boldsymbol{x}_{i+1}\right\rangle$ when $d=2$ is

$$
\mathbf{H}=\left[\begin{array}{l}
\frac{\partial^{2} \ln \left\langle\boldsymbol{b}, \boldsymbol{x}_{i+1}\right\rangle}{\left(\partial b^{(1)}\right)^{2}} \frac{\partial^{2} \ln \left\langle\boldsymbol{b}, \boldsymbol{x}_{i+1}\right\rangle}{\partial b^{(1)} \partial b^{(2)}} \\
\frac{\partial^{2} \ln \left\langle\boldsymbol{b}, \boldsymbol{x}_{i+1}\right\rangle}{\partial b^{(1)} \partial b^{(2)}} \frac{\partial^{2} \ln \left\langle\boldsymbol{b}, \boldsymbol{x}_{i+1}\right\rangle}{\left(\partial b^{(2)}\right)^{2}}
\end{array}\right]=-\left[\begin{array}{cc}
a_{1}^{2} & a_{1} a_{2} \\
a_{1} a_{2} & a_{2}^{2}
\end{array}\right],
$$

where $a_{j} \stackrel{\text { def }}{=} \frac{x_{i+1}^{(j)}-x_{i+1}^{\left(j^{\prime}\right)}}{\left\langle\boldsymbol{b}, \boldsymbol{x}_{i+1}\right\rangle}$.

TheOREM B.2 A symmetric matrix $\mathbf{A}$ is negative (positive) semidefinite if and only if all eigenvalues of $\mathbf{A}$ are nonpositive (nonnegative).

To find the eigenvalues of $\mathbf{H}$, we need to set $\operatorname{det}(\mathbf{H}-\lambda \mathbf{I})$ equal to 0 :

$$
\operatorname{det}(\mathbf{H}-\lambda \mathbf{I})=\left(-a_{1}^{2}-\lambda\right)\left(-a_{2}^{2}-\lambda\right)-\left(a_{1} a_{2}\right)^{2}=0
$$

where $\mathbf{I}$ is the identity matrix, and solve (B5) for $\lambda$. This results in the eigenvalues of $\mathbf{H}$ :

$$
\lambda_{1}=0, \quad \lambda_{2}=-\left(a_{1}^{2}+a_{2}^{2}\right)=-\left(\frac{\left(x_{i+1}^{(1)}-x_{i+1}^{(2)}\right)^{2}}{\left\langle\boldsymbol{b}, \boldsymbol{x}_{i+1}\right\rangle^{2}}+\frac{\left(x_{i+1}^{(2)}-x_{i+1}^{(1)}\right)^{2}}{\left\langle\boldsymbol{b}, \boldsymbol{x}_{i+1}\right\rangle^{2}}\right) \leq 0,
$$

which means that $\ln \left\langle\boldsymbol{b}, \boldsymbol{x}_{i+1}\right\rangle$ is concave when $d=2$ by theorem B.1 and B.2, and the eigenvalues of $\mathbf{H}$ for the general case of $d \geq 3$ are

$$
\lambda_{1}=0, \lambda_{2}=0, \ldots, \lambda_{d-1}=0, \lambda_{d}=-\sum_{j=1}^{d} a_{j}^{2} \leq 0,
$$

which means that $\ln \left\langle\boldsymbol{b}, \boldsymbol{x}_{i+1}\right\rangle$ is concave for all $d \in \mathbb{Z}_{\geq 2}$ by theorem B.1 and B.2. Consequently, $\sum_{i \in J_{n}} \ln \left\langle\boldsymbol{b}, \boldsymbol{x}_{i+1}\right\rangle$ in (4) is a concave function of $\boldsymbol{b} \in \Delta^{d-1}$ by the fact that the sum of concave (convex) functions is concave (convex).

\section{Appendix C: Concavity of transaction cost factor}

Let us check whether TCF $w_{n}$ in (21) is a concave function of $\boldsymbol{b}_{n+1} \in \Delta^{d-1}$ or not by rewriting (21) as

$$
\frac{c_{p}+c_{s}}{1+c_{p}} \sum_{l \in G_{n}}\left(\frac{b_{n}^{(l)} x_{n}^{(l)}}{\left\langle\boldsymbol{b}_{n}, \boldsymbol{x}_{n}\right\rangle}-b_{n+1}^{(l)} w_{n}\right)=1-w_{n}
$$

where the set $G_{n}$ is defined as $G_{n} \stackrel{\text { def }}{=}\left\{j \in\{1,2, \ldots, d\} \mid \frac{b_{n}^{(j)} x_{n}^{(j)}}{\left\langle\boldsymbol{b}_{n}, \boldsymbol{x}_{n}\right\rangle} \geq b_{n+1}^{(j)} w_{n}\right\}$, and $G_{n}$ has, if there is no trading at the end of the $n$-th period, the property of 
$G_{n}=\{1,2, \ldots, d\} \Leftrightarrow w_{n}=1 \Leftrightarrow b_{n+1}^{(j)}=\frac{b_{n}^{(j)} x_{n}^{(j)}}{\left\langle\boldsymbol{b}_{n}, \boldsymbol{x}_{n}\right\rangle}, \forall j$. Equation $(\mathrm{C} 1)$ can be rewritten again as

$$
w_{n}=\frac{1-\frac{c_{p}+c_{s}}{1+c_{p}} \sum_{l \in G_{n}} \frac{b_{n}^{(l)} x_{n}^{(l)}}{\left\langle\boldsymbol{b}_{n}, \boldsymbol{x}_{n}\right\rangle}}{1-\frac{c_{p}+c_{s}}{1+c_{p}} \sum_{l \in G_{n}} b_{n+1}^{(l)}} .
$$

The first-order partial derivative of $w_{n}$ with respect to $b_{n+1}^{(j)}$ (Figure 8 shows the direction of the partial derivative when $d=3$ ) is

$$
\frac{\partial w_{n}}{\partial b_{n+1}^{(j)}}=\left\{\begin{array}{cl}
\dot{w}_{n}, & \text { if } j \in G_{n} \wedge j^{\prime} \notin G_{n} \\
-\dot{w}_{n}, & \text { if } j \notin G_{n} \wedge j^{\prime} \in G_{n} \\
0, & \text { otherwise }
\end{array}\right.
$$

where

$$
\dot{w}_{n} \stackrel{\frac{c_{p}+c_{s}}{=}}{\frac{\text { def }}{1+c_{p}}\left(1-\frac{c_{p}+c_{s}}{1+c_{p}} \sum_{l \in G_{n}} \frac{b_{n}^{(l)} x_{n}^{(l)}}{\left\langle\boldsymbol{b}_{n}, \boldsymbol{x}_{n}\right\rangle}\right)}=
$$

and $j^{\prime} \stackrel{\text { def }}{=}\left\{\begin{array}{cl}d, & \text { if } j=1 \\ j-1, & \text { otherwise }\end{array}\right.$. Hence, $w_{n}$ is not differentiable with respect to $b_{n+1}^{(j)} \quad$ when a true statement among i) $j \in G_{n} \wedge j^{\prime} \notin G_{n}$, ii) $j \notin G_{n} \wedge j^{\prime} \in G_{n}$, and iii) $\left(j \in G_{n} \wedge j^{\prime} \in G_{n}\right) \vee\left(j \notin G_{n} \wedge j^{\prime} \notin G_{n}\right)$ in (C3) changes as $\boldsymbol{b}_{n+1}$ changes. For example, $w_{n}$ is not differentiable at the maximum point, $\boldsymbol{b}_{n+1}^{\star}$ in (30), since the left partial derivative:

$$
\frac{\partial_{-} w_{n}}{\partial b_{n+1}^{(j)}}=\dot{w}_{n}=\frac{\frac{c_{p}+c_{s}}{1+c_{p}}}{1-\frac{c_{p}+c_{s}}{1+c_{p}}}
$$

and the right partial derivative:

$$
\frac{\partial_{+} w_{n}}{\partial b_{n+1}^{(j)}}=-\dot{w}_{n}=-\frac{\frac{c_{p}+c_{s}}{1+c_{p}}}{1-\frac{c_{p}+c_{s}}{1+c_{p}}}
$$

are not equal. The second-order partial derivative is

$$
\frac{\partial^{2} w_{n}}{\left(\partial b_{n+1}^{(j)}\right)^{2}}= \begin{cases}\ddot{w}_{n}, & \text { if }\left(j \in G_{n} \wedge j^{\prime} \notin G_{n}\right) \vee\left(j \notin G_{n} \wedge j^{\prime} \in G_{n}\right) \\ 0, & \text { otherwise }\end{cases}
$$


where

$$
\ddot{w}_{n} \stackrel{\text { def }}{=} \frac{2\left(\frac{c_{p}+c_{s}}{1+c_{p}}\right)^{2}\left(1-\frac{c_{p}+c_{s}}{1+c_{p}} \sum_{l \in G_{n}} \frac{b_{n}^{(l)} x_{n}^{(l)}}{\left\langle\boldsymbol{b}_{n}, \boldsymbol{x}_{n}\right\rangle}\right)}{\left(1-\frac{c_{p}+c_{s}}{1+c_{p}} \sum_{l \in G_{n}} b_{n+1}^{(l)}\right)^{3}} .
$$

The second-order mixed partial derivative is

$$
\frac{\partial^{2} w_{n}}{\partial b_{n+1}^{(j)} \partial b_{n+1}^{(k)}}= \begin{cases}\ddot{w}_{n}, & \text { if }\left(j \in G_{n} \wedge j^{\prime} \notin G_{n} \wedge k \in G_{n} \wedge k^{\prime} \notin G_{n}\right) \\ & \vee\left(j \notin G_{n} \wedge j^{\prime} \in G_{n} \wedge k \notin G_{n} \wedge k^{\prime} \in G_{n}\right) \\ -\ddot{w}_{n}, & \text { if }\left(j \in G_{n} \wedge j^{\prime} \notin G_{n} \wedge k \notin G_{n} \wedge k^{\prime} \in G_{n}\right), \\ & \vee\left(j \notin G_{n} \wedge j^{\prime} \in G_{n} \wedge k \in G_{n} \wedge k^{\prime} \notin G_{n}\right) \\ 0, & \text { otherwise }\end{cases}
$$

where $k^{\prime} \stackrel{\text { def }}{=}\left\{\begin{array}{cl}d, & \text { if } k=1 \\ k-1, & \text { otherwise }\end{array}\right.$, and $j \neq k$. The concavity of $w_{n}$ in (21) at differentiable points is determined by theorem B.1 and B.2; the Hessian matrix of $w_{n}$ at differentiable points when $d=2$ is

$$
\mathbf{H}=\left[\begin{array}{cc}
\frac{\partial^{2} w_{n}}{\left(\partial b^{(1)}\right)^{2}} & \frac{\partial^{2} w_{n}}{\partial b^{(1)} \partial b^{(2)}} \\
\frac{\partial^{2} w_{n}}{\partial b^{(1)} \partial b^{(2)}} & \frac{\partial^{2} w_{n}}{\left(\partial b^{(2)}\right)^{2}}
\end{array}\right]=\left[\begin{array}{cc}
\ddot{w}_{n} & -\ddot{w}_{n} \\
-\ddot{w}_{n} & \ddot{w}_{n}
\end{array}\right] \cdot{ }^{1}
$$

To find the eigenvalues of $\mathbf{H}$, we need to set $\operatorname{det}(\mathbf{H}-\lambda \mathbf{I})$ equal to 0 :

$$
\operatorname{det}(\mathbf{H}-\lambda \mathbf{I})=\left(\ddot{w}_{n}-\lambda\right)^{2}-\ddot{w}_{n}^{2}=0
$$

This results in the eigenvalues of $\mathbf{H}$ :

$$
\lambda_{1}=0, \lambda_{2}=2 \ddot{w}_{n},
$$

where $\ddot{w}_{n}>0$ by the inequalities: $0<\frac{c_{p}+c_{s}}{1+c_{p}}<1,0 \leq \sum_{l \in G_{n}} \frac{b_{n}^{(l)} x_{n}^{(l)}}{\left\langle\boldsymbol{b}_{n}, \boldsymbol{x}_{n}\right\rangle} \leq 1$, and $0 \leq \sum_{l \in G_{n}} b_{n+1}^{(l)} \leq 1$. The eigenvalues of $\mathbf{H}$ for the general case of $d \geq 3$ are

$$
\lambda_{1}=0, \lambda_{2}=0, \ldots, \lambda_{d-1}=0, \lambda_{d}=2 \ddot{w}_{n} .
$$

Consequently, $w_{n}$ in (21) at differentiable points is convex because all the eigenvalues of the Hessian matrix are nonnegative.

However, $w_{n}$ is quasi-concave by theorem 5.4: TCF $w_{n}$ in (21) is a unimodal function of $\boldsymbol{b}_{n+1}$ because $w_{n}$ strictly decreases as $\boldsymbol{b}_{n+1}$ goes away from the maximum point $\boldsymbol{b}_{n+1}^{\star}$ in (30), as shown in Figure 5(a), 5(c), and $5(\mathrm{e}){ }^{2}$

1 Only the two cases are considered: $G_{n}=\{1\}$ and $G_{n}=\{2\}$, even though there are four cases: $G_{n}=\varnothing, G_{n}=\{1\}, G_{n}=\{2\}$, and $G_{n}=\{1,2\}$. Firstly, $G_{n}$ cannot be the empty set because of $G_{n}=\varnothing \Leftrightarrow \frac{b_{n}^{(j)} x_{n}^{(j)}}{\left\langle b_{n}, x_{n}\right\rangle}<b_{n+1}^{(j)}, \forall j$ (i.e. $\frac{b_{n}^{(j)} x_{n}^{(j)}}{\left\langle b_{n}, x_{n}\right\rangle}<b_{n+1}^{(j)}, \forall j$ is false by $\left.\boldsymbol{b}_{n}, \boldsymbol{b}_{n+1} \in \Delta^{1}\right)$. Secondly, $\boldsymbol{b}_{n+1}^{\star}$ in (30), which satisfies $\boldsymbol{b}_{n+1}=\boldsymbol{b}_{n+1}^{\star} \Leftrightarrow G_{n}=\{1,2\}$, is the non-differentiable point as mentioned in (C5) and (C6).

2 The mathematical proof of the unimodality is not provided in this paper. 
In summary, TCF $w_{n}$ in (21) is a continuous (as shown in Figure 5(b), 5(d), and 5(f)) but not differentiable and quasi-concave but not concave function of $\boldsymbol{b}_{n+1} \in \Delta^{d-1}$. 